\title{
Allais, Ellsberg, and preferences for hedging
}

\author{
MARK DEAN \\ Department of Economics, Columbia University \\ Pietro Ortoleva \\ Department of Economics, Columbia University
}

\begin{abstract}
Two of the most well known regularities observed in preferences under risk and uncertainty are ambiguity aversion and the Allais paradox. We study the behavior of an agent who can display both tendencies simultaneously. We introduce a novel notion of preference for hedging that applies to both objective lotteries and uncertain acts. We show that this axiom, together with other standard ones, is equivalent to a representation in which the agent (i) evaluates ambiguity using multiple priors, as in the model of Gilboa and Schmeidler (1989), and (ii) evaluates objective lotteries by distorting probabilities, as in the rank dependent utility model, but using the worst from a set of distortions. We show that a preference for hedging is not sufficient to guarantee Ellsberg-like behavior if the agent violates expected utility for objective lotteries; we provide a novel axiom that characterizes this case, linking the distortions for objective and subjective bets.

Keywords. Ambiguity aversion, Allais paradox, Ellsberg paradox, hedging, multiple priors, subjective mixture, probability weighting, rank dependent utility.

JEL CLASSIFICATION. D81.
\end{abstract}

\section{INTRODUCTION}

Considerable empirical and theoretical work has been devoted to studying two classes of paradoxes in individual decision making: (i) violations of von Neumann and Morgenstern's expected utility for objective risk-most notably the Allais paradox-and (ii) violations of (Savage) expected utility for subjective uncertainty-usually called uncertainty (or ambiguity) aversion, as demonstrated by the Ellsberg paradox. These behaviors constitute two of the most widely studied and robust phenomena in experimental economics and the psychology of individual decision making. Two vast theoretical literatures have emerged, each intended to generalize standard models to account for

Mark Dean: mark . dean@columbia.edu

Pietro Ortoleva: pietro.ortoleva@columbia.edu

We thank Faruk Gul, one anonymous referee, as well as Simone Cerreia-Vioglio, Eddie Dekel, David Dillenberger, Federico Echenique, Drew Fudenberg, Paolo Ghirardato, Anna Gumen, Edi Karni, Peter Klibanoff, Fabio Maccheroni, Massimo Marinacci, Efe Ok, Leonardo Pejsachowicz, Gil Riella, Kota Saito, Todd Sarver, Andrei Savochkin, Kyoungwon Seo, Marciano Siniscalchi, Peter Wakker, and the participants at various seminars and conferences for their useful comments. This work was supported by NSF Grant number SES$1156090 / 1156091$.

Copyright $\odot 2017$ The Authors. Theoretical Economics. The Econometric Society. Licensed under the Creative Commons Attribution-NonCommercial License 3.0. Available at http: //econtheory . org. DOI: 10.3982/TE1960 
one of the two phenomena. Substantially less attention, however, has been devoted to studying the relationship between the two or to the development of models that allow both behaviors simultaneously. The vast majority of models designed to explain Allaislike preferences consider only objective probabilities, whereas models that study uncertainty aversion either do not consider objective probabilities (as in the setup of Savage 1954) or, if they do, explicitly assume that the agent follows expected utility to assess them (as is typical in the setup of Anscombe and Aumann 1963). ${ }^{1}$

Despite these largely separate analyses, the idea of a connection between the two classes of behavior has been informally present for decades: loosely speaking, a decision maker (henceforth DM) who is "pessimistic" about the outcome of risky and uncertain events may display both tendencies. This conceptual connection is coupled with a technical one: both phenomena can been considered violations of some form of linearity (or independence with respect to probability mixtures) in the preferences of the agent. Recent experimental evidence also suggests that the Allais and Ellsberg paradoxes are empirically linked: Dean and Ortoleva (2017) show that subjects who display one behavior are significantly more likely to exhibit the other.

The goal of this paper is then threefold. First, we establish a conceptual link between uncertainty aversion and Allais-type behavior by showing how to jointly characterize the two using a novel axiom: a generalized notion of preference for hedging. Second, we use this axiom to derive a representation that simultaneously captures pessimism in the domains of risk and uncertainty. Third, we study the behavioral implications of doing so-specifically the relationship between preference for hedging and ambiguity aversion while allowing for non-expected utility behavior in objective lotteries.

Our main behavioral axiom is a generalized notion of hedging. Since Schmeidler (1989), the concept of preference for hedging has been used to capture uncertainty aversion by postulating that for any two acts between which she is indifferent, a DM prefers the probability mixture of the two acts to the acts themselves. We extend this idea to capture pessimism over objective lotteries by replacing probability mixtures with outcome mixtures in the style of Ghirardato et al. (2003): the outcome mixture of two outcomes is a third outcome with a utility exactly halfway between those of the original two. ${ }^{2}$ We define the mixture of two lotteries $p$ and $q$ as the set of lotteries generated using outcome mixtures considering all possible joint distributions of $p$ and $q$. A mixture of two acts is then defined pointwise.

Hedging states that for any three acts $f, g$, and $h$, if a DM is indifferent between $f$ and $g$, and $h$ can be obtained as a mixture of $f$ and $g$, then $h$ must be weakly preferred to both. When applied to lotteries, we argue that a pessimistic DM will exhibit a preference for hedging because it reduces the variance of the utility of outcomes. ${ }^{3}$ When applied to

\footnotetext{
${ }^{1}$ Exceptions are Wakker $(2001,2010)$, Klibanoff et al. (2005), and Drapeau and Kupper (2013). We refer to Section 4 for an analysis of the literature.

${ }^{2}$ For example, to mix $\$ 0$ and $\$ 10$ we look for a monetary amount that generates a utility halfway between the utility of $\$ 0$ and the utility of $\$ 10$. If the utility is linear, this would be $\$ 5$; less in case of diminishing marginal utility.

${ }^{3}$ That is, a lottery $r$ that is generated as a 50/50 mixture between $p$ and $q$ will have expected utility halfway between that of $p$ and $q$, but a variance in utilities that is less than the average variance of $p$ and $q$.
} 
acts, hedging reduces subjective uncertainty by mixing the outcomes that acts return in each state (akin to the axiom in Schmeidler 1989).

We show that our generalized preference for hedging, along with other standard axioms, characterizes a model that generalizes the maxmin expected utility (MMEU) model of Gilboa and Schmeidler (1989) to allow for Allais-style behavior. In this representation, the DM evaluates acts as in MMEU: having evaluated the outcome of the act in each state, she considers a set of priors $\Pi$ over the states of the world and takes expectations using the worst of the priors in $\Pi$. This model differs from MMEU in that objective lotteries are not assessed using expected utility. Rather, the agent distorts probabilities following a procedure similar to the rank dependent utility (RDU) model of Quiggin (1982). Here, however, she considers a set of convex (pessimistic) probability distortions and uses the worst one to evaluate the objective lottery. This is a strict generalization of RDU with convex distortion. In Section 2.3, we argue that this flexibility is desirable, as it allows us to capture forms of pessimism that RDU cannot.

An advantage of our approach is that it allows us to understand the implications of hedging under subjective uncertainty while relaxing expected utility on objective uncertainty. Despite being pessimistic (in the sense that they evaluate each act with the worst prior), our DMs need not exhibit Ellsberg paradox-type behavior. The opposite may occur if objective probabilities are "distorted" more than subjective probabilities. ${ }^{4}$ Thus, the model is compatible with the evidence of such behavior in different contexts (e.g., Tversky and Fox 1995). Although we deem such generality desirable, the special case in which behavior does not contradict the Ellsberg paradox is of natural interest, and in Section 3 we introduce a novel additional axiom that captures this case. Adding this axiom restricts the parameters in our representation, linking the distortions applied to objective and subjective bets, guaranteeing that the former are "less severe" than the latter.

Section 4 discusses the relationship between our work and the literature. There are a small number of papers that explicitly consider the relationship between Allais-like behavior and uncertainty aversion. Segal (1987a, 1990) connects the Allais and Ellsberg paradoxes by suggesting that ambiguous acts can be seen as compound lotteries. Wakker (2001) focuses on the case in which the agent's preferences take the form of Choquet expected utility for both risk and ambiguity, and shows that by generalizing the common consequence effect, one can characterize pessimism in both the objective and subjective domains. Our assumption of a generalized preference for hedging is arguably of a similar spirit, although applicable to a broader class of preferences. Ghirardato et al. (2003) show that one can obtain an MMEU-like representation on Savage acts by considering outcome mixtures and disregarding objective lotteries, thus not restricting, but also not modeling, how the agent reacts to them. ${ }^{5}$

\footnotetext{
${ }^{4}$ This possibility has been noted by Epstein (1999) and Wakker (2001). Indeed this result shows that when preferences are not expected utility for objective lotteries the well known link between preferences for hedging on the one hand and Ellsberg-like behavior on the other no longer holds as our preferences abide by the former but not the latter.

${ }^{5}$ Other results appear in Klibanoff et al. (2005) and Drapeau and Kupper (2013). We refer to Section 4 for more details.
} 
The remainder of the paper is organized as follows. Section 2 presents the formal setup, the axioms, and the representation theorem. Section 3 studies the special case in which the agent always distorts subjective probabilities more than objective ones. Section 4 discusses the relevant literature. Appendix A describes an an alternative, equivalent representation in which subjects treat objective lotteries like ambiguous prospects. Appendix B discusses how our model generates the Allais paradox. Appendix $\mathrm{C}$ describes what happens when the assumption of hedging neutrality is added to our model. Appendix D contains proofs.

\section{THE MODEL}

\subsection{Formal setup}

We consider a standard Anscombe-Aumann setup with the additional restrictions that the set of consequences is both connected and compact. Consider a finite (nonempty) set $\Omega$ of states of the world and a (nonempty) set $X$ of consequences that we assume is a connected and compact subset of a metric space. ${ }^{6}$ By $\Delta(X)$ we denote the set of simple probability measures over $X$, and $\mathcal{F}$ denotes the set of Anscombe-Aumann acts $f: \Omega \rightarrow \Delta(X)$. We metrize $\Delta(X)$ such that metric convergence on it coincides with weak convergence of Borel probability measures. We metrize $\mathcal{F}$ pointwise.

Our primitive is a complete, transitive, nondegenerate binary relation $\succeq$ on $\mathcal{F}$, the symmetric and asymmetric components of which are denoted $\sim$ and $\succ$.

We use additional standard notation. We denote by $\delta_{x} \in \Delta(X)$ the degenerate lottery (Dirac measure at $x$ ) that yields the prize $x \in X$ with certainty. For any $x, y \in X$ and $\alpha \in$ $(0,1), \alpha x+(1-\alpha) y$ is the lottery that returns $x$ with probability $\alpha$ and $y$ with probability $(1-\alpha)$. With the usual slight abuse of notation, for any $p \in \Delta(X), p$ is the constant act in $\mathcal{F}$ such that $p(\omega)=p$ for all $\omega \in \Omega$. For any $p \in \Delta(X), c_{p}$ is the certainty equivalent of $p$, i.e., an element of $X$ such that $p \sim \delta_{c_{p}}$. When $|\Omega|=1$, this setup coincides with a standard preference over von Neumann-Morgenstern (vNM lotteries), a special case of particular interest for our analysis.

We note that we use the setup of Anscombe and Aumann (1963) for convenience, as it allows for both risk and uncertainty, and because it makes comparisons with other models more direct. However, it is straightforward to translate our results into an alternative setup in which preferences are defined over the union of simple vNM lotteries on $X$ and Savage acts with consequences $X$, i.e., $\Delta(X) \cup X^{\Omega}$.

\subsection{Axioms}

We begin by imposing some basic axioms on our preference relation. To this end, we use the following standard definition of first order stochastic dominance (FOSD).

\footnotetext{
${ }^{6}$ It is standard practice to generalize our analysis to the case in which $X$ is a connected and compact topological space or to the case in which the state space is infinite. To do the latter, we would need to adapt the continuity axiom (Axiom 3): see Section 2.2, specifically the discussion after Axiom 3.

${ }^{7}$ This alternative setup might be appealing as it contains no assumptions on the timing of the resolution of uncertainty and risk (as opposed to the setup in Anscombe and Aumann 1963).
} 
Definition 1. For any $p, q \in \Delta(X)$, we say that $p$ first order stochastically dominates $q$, denoted $p \unrhd_{\text {FOSD }} q$, if $p\left(\left\{x: \delta_{x} \succeq \delta_{z}\right\}\right) \geq q\left(\left\{x: \delta_{x} \succeq \delta_{z}\right\}\right)$ for all $z \in X$. We say that $p \triangleright_{\mathrm{FOSD}} q$ if $p \unrhd_{\mathrm{FOSD}} q$ and $p\left(\left\{x: \delta_{x} \succeq \delta_{z}\right\}\right)>q\left(\left\{x: \delta_{x} \succeq \delta_{z}\right\}\right)$ for some $z \in X$.

AxIOM 1 (FOSD). For any $p, q \in \Delta(X)$, if $p \unrhd_{\mathrm{FOSD}} q$, then $p \succeq q$, and if $p \triangleright_{\mathrm{FOSD}} q$, then $p \succ q$.

Aхіом 2 (Monotonicity). For any $f, g \in \mathcal{F}$, if $f(\omega) \succeq g(\omega)$ for all $\omega \in \Omega$, then $f \succeq g$.

Axıом 3 (Continuity). The sets $\{g \in \mathcal{F}: g \succeq f\}$ and $\{g \in \mathcal{F}: g \preceq f\}$ are closed for all $f \in \mathcal{F}$.

The axioms above are standard and implied by most models. Axiom 1 imposes compliance with FOSD for objective lotteries. Axiom 2 posits that if what $f$ returns is better than what $g$ returns in every state of the world, then $f$ must be preferred to $g$. Axiom 3 is a standard continuity assumption. ${ }^{8}$

We now introduce the notion of outcome mixture that will be central to our analysis. We follow Ghirardato et al. (2003) to define "outcome mixtures" for the consequences in $X .{ }^{9}$ We will then extend this idea to the outcome mixture of lotteries and of acts.

Consider two consequences $x, y \in X$ and suppose that, in some model, the agent assigns a utility to all elements of $X$. We wish to identify the element with a utility halfway between those of $x$ and $y$. Ghirardato et al. (2003) introduce a technique that allows us to elicit this element for any continuous utility function, as long as preferences are well behaved (in a sense discussed below). We will adapt their technique, originally developed for Savage acts, to the case of objective lotteries.

Definition 2. For any $x, y \in X$, if $\delta_{x} \succeq \delta_{y}$, we say that $z \in X$ is a $\frac{1}{2}$-mixture of $x$ and $y$, if $\delta_{x} \succeq \delta_{z} \succeq \delta_{y}$ and

$$
\frac{1}{2} x+\frac{1}{2} y \sim \frac{1}{2} c_{\frac{1}{2} x+\frac{1}{2} z}+\frac{1}{2} c_{\frac{1}{2} z+\frac{1}{2} y} .
$$

We denote $z$ by $\frac{1}{2} x \oplus_{\succeq} \frac{1}{2} y \cdot{ }^{10}$

Consider some $x, y, z \in X$ such that $\delta_{x} \succeq \delta_{z} \succeq \delta_{y}$ and (1) holds. The lottery on the left is simply a $50 \%$ chance of $x$ and a $50 \%$ chance of $y$. The lottery on the right is a $50 \%$ chance of two other outcomes: the first is the certainty equivalent of a $50 \%$ chance of $x$ and a $50 \%$ chance of $z$; the second is the certainty equivalent of a $50 \%$ chance of $z$ and

\footnotetext{
${ }^{8}$ To extend our analysis to an infinite state space we would have to adapt Axiom 3 (continuity) and require (i) $\{\alpha \in[0,1]: \alpha f+(1-\alpha) g \succeq h\}$ and $\{\alpha \in[0,1]: h \succeq \alpha f+(1-\alpha) g\}$ are closed, and (ii) $\{q \in \Delta(X): q \succeq p\}$ and $\{q \in \Delta(X): q \preceq p\}$ are closed for all $p \in \Delta(X)$. We would then obtain representations identical to ours, but in which the measures over $\Omega$ are just finitely additive. Adding Arrow's monotone continuity axiom would then guarantee countable additivity (see Chateauneuf et al. 2005).

${ }^{9}$ The idea of using one element of the outcomes as an alternative to probability mixtures was, to our knowledge, introduced by Gul (1992). Similar approaches appear in Chew and Karni (1994), Wakker (1994), Casadesus-Masanell et al. (2000a, 2000b), and Köbberling and Wakker (2003). See Section 4 for more.

${ }^{10}$ For simplicity of notation, in most of the following discussion we drop the subscript and denote the mixture above only by $\oplus$.
} 
a 50\% chance of $y$. Under expected utility, a DM will be indifferent between them if and only if $z$ has a utility exactly halfway between those of $x$ and $y$. Ghirardato et al. (2003) show that this is also true for all preferences in the much broader class of "locally biseparable" preferences-essentially, those for which a cardinally unique utility function can be identified.

Because $X$ is connected and preferences are continuous, such an outcome mixture will always exist. Furthermore, we can extend this definition to any other mixture $\lambda x \oplus(1-\lambda) y$ for any dyadic rational $\lambda \in(0,1)$ by simply iteratively applying the above definition. ${ }^{11}$ We refer to Ghirardato et al. $(2001,2003)$ for further discussion.

Although the element $\frac{1}{2} x \oplus \frac{1}{2} y$ is always well defined in our setting, its interpretation presumes the existence of a utility function that is, in some sense, cardinally unique. We now provide a necessary and sufficient condition to guarantee that this is the case. Consider some $x, y, z^{\prime}, z^{\prime \prime}$ such that $z^{\prime}$ and $z^{\prime \prime}$ are "in between" $x$ and $y$ according to $\succeq$. Then consider the two lotteries

$$
\frac{1}{2} c_{\frac{1}{2} x+\frac{1}{2} z^{\prime}}+\frac{1}{2} c_{\frac{1}{2} y+\frac{1}{2} z^{\prime \prime}} \quad \text { and } \quad \frac{1}{2} c_{\frac{1}{2} x+\frac{1}{2} z^{\prime \prime}}+\frac{1}{2} c_{\frac{1}{2} y+\frac{1}{2} z^{\prime}}
$$

In the former lottery, $x$ is mixed with $z^{\prime}$ and $y$ with $z^{\prime \prime}$, and then they are mixed together; in the latter, $x$ is mixed first with $z^{\prime \prime}$ and $y$ is mixed with $z^{\prime}$, and then they are mixed together. In both cases, the only weight involved is $\frac{1}{2}$, and $x$ is always mixed with some element worse than it, whereas $y$ is mixed with some element better than it. The only difference is in the "order" of this mixture. The following axiom guarantees that the agent is indifferent between these two lotteries; she should not care about this order. This axiom is an adaptation of the $E$-substitution axiom in Ghirardato et al. (2001) to the case of objective lotteries.

Aхіом 4 (Objective trade-off consistency). Consider any $x, y, z^{\prime}, z^{\prime \prime} \in X$ such that $\delta_{x} \succeq$ $\delta_{z^{\prime}} \succeq \delta_{z^{\prime \prime}} \succeq \delta_{y}$, and $c_{\frac{1}{2} r+\frac{1}{2} s}$ exists for $r=x, y$ and $s=z^{\prime}, z^{\prime \prime}$. Then we have

$$
\frac{1}{2} c_{\frac{1}{2} x+\frac{1}{2} z^{\prime}}+\frac{1}{2} c_{\frac{1}{2} y+\frac{1}{2} z^{\prime \prime}} \sim \frac{1}{2} c_{\frac{1}{2} x+\frac{1}{2} z^{\prime \prime}}+\frac{1}{2} c_{\frac{1}{2} y+\frac{1}{2} z^{\prime}}
$$

Axiom 4 is clearly satisfied under expected utility, as it is a weakening of independence on objective lotteries (under which both would be indifferent to a lottery that returns each outcome with probability $\frac{1}{4}$ ). However, it holds for a much broader class of preferences: for example, it is satisfied by an agent who evaluates each lottery of the form $\frac{1}{2} a+\frac{1}{2} b$, where $\delta_{a} \succeq \delta_{b}$, by $\gamma\left(\frac{1}{2}\right) u(a)+\left(1-\gamma\left(\frac{1}{2}\right)\right) u(b)$, where $\gamma\left(\frac{1}{2}\right)$ could be any number between 0 and 1 . Thus, Axiom 4 is compatible with even extreme forms of probability weighting and is satisfied by many generalizations of expected utility suggested in the literature-from RDU to Gul's (1991) disappointment aversion.

\footnotetext{
${ }^{11}$ Any $\lambda \in[0,1]$ is dyadic rational if for some finite $N$, we have $\lambda=\sum_{i=1}^{N} a_{i} / 2^{i}$, where $a_{i} \in\{0,1\}$ for every $i$ and $a_{N}=1$. Then we use $\lambda x \oplus(1-\lambda) y$ as shorthand for the iterated preference average $\frac{1}{2} z_{1} \oplus \frac{1}{2}\left(\cdots\left(\frac{1}{2} z_{N-1} \oplus\right.\right.$ $\left.\left.\frac{1}{2}\left(\frac{1}{2} z_{N} \oplus \frac{1}{2} y\right)\right) \cdots\right)$, where for every $i, z_{i}=x$ if $a_{i}=1$ and $z_{i}=y$ otherwise. Alternatively, we could have defined $\lambda x \oplus \lambda y$ for any real number $\lambda \in(0,1)$ by defining it for dyadic rationals first, and then using continuity of the preferences to define it for the whole $[0,1]$. The two approaches are clearly identical in our axiomatic structure; we choose to use the most restrictive definition to state the axioms in the weakest form of which we are aware.
} 
Following the literature, it is straightforward to show that Axioms 1-4 are sufficient to guarantee that we can find a utility representation that is cardinally unique, thus allowing us to interpret $\oplus$ in a meaningful way. (See, for example, Lemma 1 in Ghirardato et al. 2001.)

We now extend outcome mixtures to mixtures of lotteries and acts, one of the key contributions of our paper and an essential step in defining the notion of hedging at the core of our analysis. We begin by extending the concept to mixtures of lotteries. Consider $p=\frac{1}{2} x+\frac{1}{2} y$ and $q=\frac{1}{2} z+\frac{1}{2} w$, where $\delta_{x}, \delta_{z} \succ \delta_{y}, \delta_{w}$. If we assume a joint distribution between them, we can construct a mixture of these two lotteries using outcome mixtures. For example, if we imagine that $p$ always pays out $x$ when $q$ pays out $z$, we can construct a lottery $r$ that half of the time pays out $\frac{1}{2} x \oplus \frac{1}{2} z$ (the prize with utility halfway between those of $x$ and $z$ ) and half of the time pays out $\frac{1}{2} y \oplus \frac{1}{2} w$. We would describe $r$ as a mixture of $p$ and $q$.

The above description is clearly only one of many possible ways to combine $p$ and $q$, assuming a particular joint distribution. Intuitively, as $\delta_{x}, \delta_{z} \succ \delta_{y}, \delta_{w}$, we have combined the two lotteries as if they were positively correlated. Alternatively, we could combine $x$ with $w$ and $y$ with $z$-i.e., treat them as negatively correlated-and obtain $\frac{1}{2}\left(\frac{1}{2} x \oplus \frac{1}{2} w\right)+\frac{1}{2}\left(\frac{1}{2} y \oplus \frac{1}{2} z\right)$. We could also combine them in many other ways depending on the assumptions regarding their joint distribution. Thus, for any two lotteries, we have a set of possible mixtures.

More generally, we can regard any two lotteries $p$ and $q$ as random variables on some underlying measure space, $V_{p}$ and $V_{q}$. There are many ways to construct such random variables, each of which would entail the same marginal distribution over prizes for $V_{p}$ and $V_{q}$ separately but would generate different joint distributions of their returns. For any given joint distribution, we can construct a third random variable that returns prizes with utilities that are exactly the mixture of the utilities of the prizes returned by the original lotteries-the random variable $\frac{1}{2} V_{p} \oplus \frac{1}{2} V_{q}$. This, in turn, defines a lottery $r \in$ $\Delta(X)$. Depending on the joint distribution of $V_{p}$ and $V_{q}$, we obtain different lotteries that constitute the set of mixtures between $p$ and $q .{ }^{12}$ We define $p \oplus q$ as the set of all such mixtures for all possible joint distributions. ${ }^{13}$

This concept of mixing of objective lotteries is similar to the standard notion of mixing of two financial assets to create a portfolio, with two substantial differences. First, we make no assumptions on the joint distribution of the lotteries, and thus we obtain the set of possible portfolios-one for each joint distribution. Second, here the mixture takes place in utilities, rather than in the prizes: thus a mixture between $\$ 10$ and $\$ 0$ is not necessarily $\$ 5$; instead, the monetary amount yields utility halfway between those of $\$ 10$

\footnotetext{
${ }^{12}$ We thank Fabio Maccheroni for suggesting this interpretation.

${ }^{13} \mathrm{~A}$ more direct construction would proceed as follows. Consider any lottery $p$ and $q$, and notice that, because both are simple lotteries, we could always find some $x_{1}, \ldots, x_{n}, y_{1}, \ldots, y_{n} \in X$, and some $\gamma_{1}, \ldots, \gamma_{n} \in[0,1]$ such that $p=\sum_{i=1}^{n} \gamma_{i} \delta_{x_{i}}$ and $q=\sum_{i=1}^{n} \gamma_{i} \delta_{y_{i}}$. (For example, the lotteries $p=\frac{1}{2} x+\frac{1}{2} y$ and $q=\frac{1}{3} z+\frac{2}{3} w$ could be both written as $p=\frac{1}{3} x+\frac{1}{6} x+\frac{1}{6} y+\frac{1}{3} y$ and $q=\frac{1}{3} z+\frac{1}{6} w+\frac{1}{6} w+\frac{1}{3} w$.) Then the set $p \oplus q$ will be the set of all combinations $r$ such that $r=\sum_{i=1}^{n} \gamma_{i}\left(\frac{1}{2} x_{i} \oplus \frac{1}{2} y_{i}\right)$. That is, we have $p \oplus q:=$ $\left\{r \in \Delta(X): \exists x_{1}, \ldots, x_{n}, y_{1}, \ldots, y_{n} \in X, \gamma_{1}, \ldots, \gamma_{n} \in[0,1]\right.$ such that $p=\sum_{i=1}^{n} \gamma_{i} \delta_{x_{i}}, q=\sum_{i=1}^{n} \gamma_{i} \delta_{y_{i}}$, and $r=$ $\left.\sum_{i=1}^{n} \gamma_{i} \delta_{\left(\frac{1}{2} x_{i} \oplus \frac{1}{2} y_{i}\right)}\right\}$
} 
and $\$ 0$. This latter point has an important implication. In finance, a portfolio comprising assets of equal expected value will have the same expected value but (weakly) lower variance in monetary terms. Thus, a risk-averse agent may strictly prefer the portfolio to the underlying assets. Instead, under our definition, the mixture of two lotteries of equal expected utility will lead to a lottery with the same expected utility but a weakly lower variance in utility terms.

Finally, we define outcome mixtures for acts. We do so pointwise: an act $h$ is a $\frac{1}{2}$ mixture between two acts $f$ and $g$ if $h(\omega) \in f(\omega) \oplus g(\omega)$ for all $\omega \in \Omega$; that is, if for every state, it returns a lottery that is a mixture between the lotteries returned by $f$ and $g$. We denote $f \oplus g$ as the set of all such mixtures of two acts.

We are now ready to introduce the main axiom of this paper.

Axiom 5 (Hedging). For any $f, g \in \mathcal{F}$ and for any $h \in f \oplus g$, if $f \sim g$, then $h \succeq f$.

We argue that this axiom captures pessimism about both risk and uncertainty. Let us begin with the former. Consider two lotteries $p$ and $q$ such that $p \sim q$, and a lottery $r$ that could be obtained as a mixture between $p$ and $q$, i.e., $r \in p \oplus q$.

Note that any expected utility (EU) agent should be neutral to this form of hedging, i.e., should rank $r \sim p \sim q$. For an expected utility maximizer, $p \sim q$ only if the expected utility of $p$ is equal to that of $q$. Therefore, $r$ must have the same expected utility but will have weakly lower variance in utilities than either $p$ or $q$. While a risk-averse EU agent might prefer to reduce the variance in dollar amounts, she must be neutral to variance in utilities because the EU functional is linear in utilities. ${ }^{14}$

By contrast, we propose that an agent who violates EU, as suggested by the Allais paradox, will not be neutral to this form of hedging. Almost by definition, such an agent dislikes exposure to risk to a greater extent than what can be explained by the curvature of the utility function and must also therefore be averse to the variance in utilities. Our agent should thus (weakly) prefer $r$ to $p$ or $q$, irrespective of how $r$ is constructed: just as in finance, a risk-averse EU agent who is indifferent between two portfolios must weakly prefer a mixture between them, irrespective of their correlation; here, an Allaislike agent should weakly prefer to hedge in utilities between two lotteries, irrespective of construction.

As an example, consider a lottery $p$ that returns $\$ 10$ and $\$ 0$ with probability $\frac{1}{2}$. For simplicity, consider its mixtures with an identical lottery. If we construct the mixture treating the two lotteries as perfectly positively correlated, we again obtain the original lottery, indifferent to itself. However, if we construct the mixture assuming that they are negatively correlated-when one returns $\$ 10$, the other returns $\$ 0$ - we obtain a degenerate lottery that returns, with probability 1 , the dollar amount $\frac{1}{2} \$ 10 \oplus \frac{1}{2} \$ 0$. Thus,

\footnotetext{
${ }^{14}$ In fact, as we analyze in Appendix C, there is a sense in which expected utility could be defined by such neutrality to hedging, in a fairly weak sense. To see it in the algebra, recall that (footnote 13) we must be able to find some $x_{1}, \ldots, x_{n}, y_{1}, \ldots, y_{n} \in X, \exists \gamma_{1}, \ldots, \gamma_{n} \in[0,1]$ such that $p=\sum_{i=1}^{n} \gamma_{i} \delta_{x_{i}}, q=\sum_{i=1}^{n} \gamma_{i} \delta_{y_{i}}$, and $r=\sum_{i=1}^{n} \gamma_{i} \delta_{\left(\frac{1}{2} x_{i} \oplus \frac{1}{2} y_{i}\right)}$. Under expected utility we must have that $u\left(\frac{1}{2} x_{i} \oplus \frac{1}{2} y_{i}\right)=\frac{1}{2} u(x)+\frac{1}{2} u(y)$, which means that if the expected utility of $p$ and of $q$ are the same, so must be that of $r$.
} 
mixing has removed all variance in utilities. While an expected utility agent will be indifferent to such a change- the expected utility of $p$ is exactly that of $\frac{1}{2} \$ 10 \oplus \frac{1}{2} \$ 0$ - a pessimistic agent should appreciate this reduced variance and be attracted to this degenerate lottery. More generally, mixing any lottery $p$ with itself returns a lottery that is more concentrated toward the mean in utility terms. A pessimistic agent should prefer this reduction in exposure and thus exhibit a preference for hedging.

Our notion of hedging is related to other notions of pessimism over objective lotteries that that have been suggested by the literature. As the next section clarifies, within the context of RDU preferences, the hedging axiom requires that weighting functions be convex (i.e., pessimistic). ${ }^{15}$

We can easily extend this argument to hedging between acts. For simplicity, consider two nondegenerate acts $f, g \in \mathcal{F}$ such that $f \sim g$, and $f(\omega)$ and $g(\omega)$ are degenerate lotteries for all $\omega$. Now, consider some $h \in f \oplus g$, and notice that $h(\omega)=\delta_{\frac{1}{2} f(\omega) \oplus \frac{1}{2} g(\omega)}$. Because no lotteries are involved, going from $f$ and $g$ to $h$ does not affect the exposure to risk: in either case, there is none. However, it will reduce the exposure to uncertainty: this is precisely the idea of the original hedging axiom of Schmeidler (1989). ${ }^{16}$ An agent who is not uncertainty seeking would then (weakly) prefer hedging, and she will rank $h$ at least as highly as $f$ and $g$.

While the hedging axiom imposes a preference for hedging for both risk and uncertainty, it posits no constraints on whether this preference should be stronger in one of the two environments. It is possible for a DM to exhibit pessimism only in the risk domain or only in the uncertainty domain: the axiom could equivalently be written as two separate axioms, one for risk and one for uncertainty. ${ }^{17}$ This observation will be relevant for our future discussion of our model's implications for ambiguity-averse behavior.

Our final axiom is the translation of the idea of the certainty-independence axiom of Gilboa and Schmeidler (1989) to our setup: ${ }^{18}$ when two acts are mixed with a neutral element, their ranking should not change. In contrast to certainty independence, however, the neutral element is not a constant act but a degenerate lottery, which is neutral regarding both risk and uncertainty. We again employ outcome mixtures because our agent could have nonlinear reactions to probability mixtures. ${ }^{19}$

\footnotetext{
${ }^{15} \mathrm{Gumen}$ et al. (2014) introduce a general notion of pessimism for preferences under risk. They show (Gumen et al. 2014, p. 22) how our hedging axiom coincides with their notion of pessimism within the context of biseparable EU preferences.

${ }^{16}$ In fact, if we impose independence for elements of $\Delta(X)$, then a preference for hedging in outcome mixtures is identical to preference for hedging in probabilities. In turn, when applied only to acts that map to degenerate lotteries, this is precisely the axiom suggested in Ghirardato et al. (2003).

${ }^{17}$ In particular, it would be equivalent to (i) for any $p, q \in \mathcal{F}$ and for any $r \in p \oplus q$, if $p \sim q$, then $r \succeq p$, and (ii) for any $f, g, h \in \mathcal{F}$ such that $f(\omega)$ and $g(\omega)$ are degenerate lotteries, if $f \sim g$ and $h(\omega)=\frac{1}{2} f(\omega) \oplus \frac{1}{2} g(\omega)$, then $h \succeq f$. The latter coincides with the postulate in Ghirardato et al. (2003).

${ }^{18} \mathrm{~A}$ preference relation satisfies certainty indepedence if for any $f, g \in \mathcal{F}$ and for any $p \in \Delta(X)$ and $\lambda \in$ $(0,1)$, we have $f \succeq g$ if and only if $\lambda f+(1-\lambda) p \succeq \lambda g+(1-\lambda) p$.

${ }^{19}$ This axiom is strictly weaker than certainty independence, as the latter implies standard independence on constant acts, which in turn implies that probability mixtures and outcome are indifferent for her: we must have $\lambda f+(1-\lambda) \delta_{x} \sim \lambda f \oplus(1-\lambda) \delta_{x}$ for all $f \in \mathcal{F}, x \in X$, and $\lambda \in(0,1)$. But then certainty independence would naturally imply Axiom 6 .
} 
Axıом 6 (Degenerate independence (DI)). For any $f, g \in \mathcal{F}$, dyadic $\lambda \in(0,1)$, and for any $x \in X$,

$$
f \succeq g \quad \Leftrightarrow \quad \lambda f \oplus(1-\lambda) \delta_{x} \succeq \lambda g \oplus(1-\lambda) \delta_{x} .
$$

\subsection{The multiple priors-multiple weighting representation}

We are now ready to discuss our representation. To this end, it will be useful to recall the RDU model for preferences over lotteries in $\Delta(X)$.

Definition 3. We say that a function $\psi:[0,1] \rightarrow[0,1]$ is a probability weighting if it is increasing and $\psi(0)=0, \psi(1)=1$. For every nonconstant function $u$ and every probability weighting $\psi$, we say that a function from $\Delta(X)$ to $\mathbb{R}$ is a (RDU) function with utility $u$ and weight $\psi$, denoted $\mathrm{RDU}_{u, \psi}$, if, for any enumeration of the elements of the support of $p$ such that $x_{i-1} \succeq x_{i}$ for $i=2, \ldots,|\operatorname{supp}(p)|$, we have

$$
\operatorname{RDU}_{u, \psi}(p):=\psi\left(p\left(x_{1}\right)\right) u\left(x_{1}\right)+\sum_{i=2}^{n}\left[\psi\left(\sum_{j=1}^{i} p\left(x_{j}\right)\right)-\psi\left(\sum_{j=1}^{i-1} p\left(x_{j}\right)\right)\right] u\left(x_{i}\right) .
$$

The main feature of the RDU model is that the DM follows a procedure similar to expected utility, except that she distorts the cumulative probability distribution of each lottery using a probability weighting function. The RDU model has many desirable properties, such as preserving continuity (provided that the probability weighting is continuous) and FOSD. Depending on the shape of $\psi$, moreover, it allows for attraction or aversion to certainty: the former occurs when $\psi$ is convex, leading to Allais-like behavior; the opposite occurs when $\psi$ is concave. When $\psi$ is linear, it coincides with expected utility. ${ }^{20}$ The RDU model is arguably the most well known non-expected utility model for objective lotteries. The cumulative prospect theory model of Tversky and Kahneman (1992), for example, is based on its framework.

In our representation, objective lotteries are assessed using a generalization of the RDU model, while acts are then assessed in a manner similar to the MMEU model.

Definition 4. Consider a complete and nondegenerate preference relation $\succeq$ on $\mathcal{F}$. We say that $\succeq$ admits a multiple priors-multiple weighting (MP-MW) representation $(u, \Pi, \Psi)$ if there exists a continuous utility function $u: X \rightarrow \mathbb{R}$, a convex and compact set of probability measures $\Pi$ on $\Omega$, and a convex, (pointwise) compact set of differentiable and convex probability weightings $\Psi$ such that $\succeq$ is represented by the functional

$$
V(f):=\min _{\pi \in \Pi} \int_{\Omega} \pi(\omega) U(f(\omega)) \mathrm{d} \omega,
$$

where $U: \Delta(X) \rightarrow \mathbb{R}$ is defined as

$$
U(p):=\min _{\psi \in \Psi} \operatorname{RDU}_{u, \psi}(p) .
$$

\footnotetext{
${ }^{20}$ See Quiggin (1982), and also Wakker (1994), Nakamura (1995), Chateauneuf (1999), Starmer (2000), Wakker (2001, 2010), Abdellaoui (2002), Köbberling and Wakker (2003), and the many references therein.
} 
We say that $\Psi$ is minimal if there is no $\Psi^{\prime} \subset \Psi$ such that the same preferences can be represented by a multiple priors-multiple weighting representation $\left(u, \Pi, \Psi^{\prime}\right)$.

THEOREM 1. Consider a complete and nondegenerate preference relation $\succeq$ on $\mathcal{F}$. The following statements are equivalent:

(i) The relation $\succeq$ satisfies Axioms 1-6.

(ii) There exists a multiple priors-multiple weighting representation $(u, \Pi, \Psi)$ of $\succeq$.

Moreover, $u$ is unique up to a positive affine transformation, $\Pi$ is unique, and there exists a unique minimal $\Psi$.

In a multiple priors-multiple weighting representation, the DM is endowed with three elements: a utility function $u$, a set of priors $\Pi$ over the states in $\Omega$, and a set of convex probability weightings $\Psi$. With respect to uncertainty, her behavior is conceptually identical to the maxmin expect utility model of Gilboa and Schmeidler (1989): she has a set of priors $\Pi$ on the states of the world, and she uses the worst one of them to aggregate the utilities returned in each state. The model differs from MMEU in the evaluation of objective lotteries. Instead of following vNM expected utility, the agent has a set of convex probability weightings $\Psi$, and she uses the worst one of those in an RDU functional to evaluate objective lotteries.

Because each of the probability weightings in $\Psi$ is convex and the agent uses the worst one to evaluate the lottery at hand, the agent will have pessimistic evaluations of objective lotteries, generating violations of expected utility in line with the Allais paradox. In particular, as we show in Appendix B, the model is always weakly compatible with a generalized version of Allais' common consequences effect, while ruling out contrary violations. $^{21}$

In the case of only objective risk, i.e., when $|\Omega|=1$, our model is a strict generalization of RDU with convex distortions. We argue that this additional flexibility could be desirable. Consider the lotteries $p=\frac{1}{3} \$ 0+\frac{1}{3} \$ 1+\frac{1}{3} \$ 10,000$ and $q=\frac{1}{3} \$ 0+\frac{1}{3} \$ 9,999+\frac{1}{3} \$ 10,000$. Under RDU, the agent must use the same probability distortion for both $p$ and $q$ : the rank of the three outcomes is the same, and because in RDU only the relative rank matters, the probability distortion is restricted to be the same. ${ }^{22}$ Specifically, under RDU, the agent distorts the intermediate outcome in the same way, despite the fact that in $p$ this intermediate outcome is comparably very bad, while in $q$ it is comparably very

\footnotetext{
${ }^{21}$ In particular, for some prize $\$ z$, and probabilities $q$ and $r$ such that $q+r<1$, find the amount $\$ x$ such that $\delta_{z} \sim r \$ 0+q \$ z+(1-q-r) \$ x$ and the amount $\$ y$ such that $q \$ 0+(1-q) \$ z \sim(r+q) \$ 0+(1-q-r) \$ y$. The common consequence effect implies that $x>y$. (The standard Allais' version uses $r=0.01$ and $q=0.89$.) Intuitively, the DM exhibits a preference for certainty. Segal (1987b) showed that, in the context of the RDU model, convexity of the weighting function is necessary and sufficient for a DM to (weakly) exhibit the common consequence effect. In Appendix B we show that the latter holds also for the multiple convex weightings case: In problems of the above form, a DM who behaves in line with the MP-MW model can have $x>y$, but cannot have $y>x$. We note also that our model does not, however, guarantee that the DM will always exhibit the common ratio effect. In the context of the RDU model, convex probability weighting is not enough to guarantee this, and thus the same must hold for our model.

${ }^{22}$ In fact, this is one of the characterizing features of RDU: see Diecidue and Wakker (2001).
} 
good. By contrast, the MP-MW model allows for the case in which in $p$ both the probabilities of $\$ 0$ and of $\$ 1$ are substantially overweighted and the probability of $\$ 10,000$ is underweighted; while for $q$, only the probability of $\$ 0$ is overweighted and those of both $\$ 9,999$ and $\$ 10,000$ are underweighted-a behavior that we believe better accords with intuitive notions of pessimism.

While our model is a generalization of the RDU model with convex weighting, it does not allow for other weighting functions, including either concave or S-shaped probability weightings (the latter of which have received empirical support from, for example, Gonzalez and Wu 1999). This is a direct implication of our application of hedging (and therefore pessimism) to all lotteries. ${ }^{23}$ In fact, one can think of the relationship between the multiple weighting model and RDU for objective risk as similar to that between MMEU and the Choquet expected utility (CEU) model of Schmeidler (1989) for subjective acts. ${ }^{24}$

Three special cases of our representations are of particular interest: first, when $\Psi$ contains only the identity function, the models coincide with MMEU (with continuous Bernoulli utility); second, when $\Pi$ is a singleton, which precludes uncertainty aversion but allows for distortions of objective lotteries; third, when $\Psi$ is a singleton but differs from the identity function, and thus the ranking of objective lotteries coincides with RDU. Clearly, the first two could be obtained by imposing forms of "hedging neutrality." For brevity, we discuss these conditions in Appendix C, where we show that the first case can be characterized by assuming that objective and subjective mixtures coincide, at least locally. To characterize the case in which $\Psi$ is a singleton, it suffices to add one of the axioms suggested in the literature to guarantee that the preferences are of the RDU form for objective lotteries; for example, the probability trade-off consistency axiom of Abdellaoui (2002) or some of the equivalents of Schmeidler's (1989) axiom of co-monotonic independence, e.g., both the co-monotonic sure-thing principle and the co-monotonic mixture independence axioms of Chateauneuf (1999). ${ }^{25}$

We conclude by noting that in the representation above, there is a sense in which the DM could be considered uncertainty averse: when $|\Pi|>1$, the agent has a set of priors and uses the worst one of those to judge uncertain events. However, this does not imply that the agent will exhibit the Ellsberg paradox: both Ellsberg behavior and its opposite are compatible with our representations, even if $|\Pi|>1$.

To see why, consider an urn with 100 balls that can be red or black, in unknown proportions. An experimenter will extract a ball from this urn, and the color of the extracted

\footnotetext{
${ }^{23}$ One could adapt our approach to allow for S-shaped weighting functions by restricting the class of lotteries to which the hedging axiom is applied, in the manner of (for example) Diecidue et al. (2009).

${ }^{24}$ Recall that CEU satisfies Schmeidler's (1989) axiom of co-monotonic independence, but need not satisfy its uncertainty aversion axiom, while MMEU satisfies the latter, but may violate the former. Similarly, RDU satisfies a version of co-monotonic independence for risk, but in its general form allows for distortions of many kinds, not only pessimistic ones. By contrast, our model may violate the equivalent of comonotonic independence, but restricts attention to pessimistic distortions.

${ }^{25}$ In turn, this implies that our axioms (esp. hedging) together with those that characterize RDU, imply the attraction for certainty axiom of Chateauneuf (1999), or probabilistic risk aversion as defined in Abdellaoui (2002), or the pessimism condition of Wakker (2001), since they are all implied by the existence of a convex RDU representation.
} 
ball determines the state of the world, $R$ or $B$. Consider the following three acts: betting on $R$ (i.e., receiving $\$ 10$ if a red ball is extracted, $\$ 0$ otherwise), betting on $B$, and an objective lottery that pays $\$ 10$ or $\$ 0$ with equal probability. Assume that the DM is indifferent between betting on red or black. The typical Ellsberg behavior is that the DM strictly prefers the objective lottery to either of the bets. Indeed, the MP-MW model allows this: for example, if $|\Pi|>1$ and $\Psi$ contains only the identity function, the model coincides with MMEU, which is compatible with Ellsberg behavior. However the MPMW model is also compatible with the opposite behavior: if $\Pi=\{(0.5,0.5)\}$ and $\Psi$ is not equal to the identity function, the agent will prefer either bet to the objective lottery, as she distorts objective probabilities but not subjective ones. ${ }^{26}$

The MP-MW model does not guarantee Ellsberg-like behavior because it does not posit that the agent should be "more pessimistic" for subjective bets than she is for objective ones: there is no relationship between the sets $\Pi$ and $\Psi$. This is a direct consequence of the fact that our main axiom, hedging, imposes no restriction on the relative pessimism of the agent between subjective and objective bets, and, as noted in Wakker (2001), one should regard Ellsberg-type behavior as the product of relative, rather than absolute, pessimism. ${ }^{27}$ In other words, our axiom imposes a generalized preference for hedging, but our theorem shows that this is insufficient to guarantee Ellsberg-like behavior in general. When preferences violate expected utility on objective lotteries, then the well known link between preferences for hedging, on the one hand, and Ellsberg-like behavior, on the other, no longer holds. ${ }^{28}$

We consider this flexibility desirable, as it allows the model to be compatible with the experimental evidence documenting behavior opposite that of Ellsberg in some environments (e.g., Tversky and Fox 1995). However, the special case in which behavior is restricted to never violating the Ellsberg paradox is of natural interest. This is discussed and characterized axiomatically, in Section 3.

\subsection{Proof sketch of Theorem 1}

In what follows, we sketch the proof of Theorem 1 . We omit many details and focus only on the sufficiency of the axioms. A complete proof appears in Appendix D.

\footnotetext{
${ }^{26}$ A similar result could be obtained also when $|\Pi|>1$, as long as the distortions of objective probabilities are stronger than those of subjective ones.

${ }^{27}$ In light of this discussion and to avoid confusion, we shall call uncertainty averse an agent who exhibits a preference for hedging - as in our model—but who may (or may not) exhibit the opposite of Ellsberg behavior. We will call ambiguity averse an agent who never does. See Section 3 for a more formal definition. (This is in line with Wakker 2001, Section 6; see also Ghirardato 2004 for more discussion.)

${ }^{28}$ This is all the more explicit if we consider what exactly the hedging axiom does in the proof of Theorem 1. In a nutshell, what we do is that we map objective lotteries into acts on a fictitious state space $[0,1]$. Thus, we map the Anscombe-Aumann setup to the space of acts on $\Omega \times[0,1]$. Then we show that the hedging axiom implies that the preferences over these acts satisfy uncertainty aversion, where mixtures are performed in utilities. That is, the hedging axiom implies a generalized preference for hedging on both parts of the state space, $\Omega$ and $[0,1]$. We then proceed to obtain the multiple prior representation in each dimension. In this sense, the hedging axiom posits a very symmetric condition on the two parts of the state space, treating them in a similar manner. However, it does not posit anything on the relative preference for hedging in each dimension, and thus does not constrain the sets of priors involved.
} 
Step 1. Map acts $\Omega \rightarrow \Delta(X)$ to acts $\Omega \times[0,1] \rightarrow X$ and obtain a derived relation $\succeq^{\prime}$. Consider the extended state space $\Omega \times[0,1]$ and call $\mathcal{F}^{\prime}$ the (Savage) acts on this space, i.e., the set of functions from $\Omega \times[0,1]$ to $X$ with the appropriate measurability. ${ }^{29}$ We can map each of the Anscombe-Aumann acts in $\mathcal{F}$ to acts in $\mathcal{F}^{\prime}$ as follows: each $f \in \mathcal{F}$ is mapped to the acts $f^{\prime}$ in $\mathcal{F}^{\prime}$ such that, for each state $\omega$ and prize $x$, the probability of receiving $x$ in state $\omega$ according to $f, f(\omega)(x)$, is equal to the Lebesgue measure of the states in $[0,1]$ that return $x$ according to $f^{\prime}$ assuming that the other component is $\omega$. We then derive a preference $\succeq^{\prime}$ on $\mathcal{F}^{\prime}$ from the original preference $\succeq$ on $\mathcal{F}$. It can be shown that $\succeq^{\prime}$ is complete, transitive, reflexive, and continuous.

Step 2. The relation $\succeq^{\prime}$ satisfies local bi-separability, and is convex and c-independent with respect to $\oplus$. Consider the event $A=\Omega \times\left[0, \frac{1}{2}\right]$ and define $\Sigma_{A}:=\left\{\varnothing, A, A^{C}, \Omega \times\right.$ $[0,1]\}$. Axiom 4 (together with Axioms 1-3) implies that the restriction of $\succeq^{\prime}$ on acts measurable in $\Sigma_{A}$ is bi-separable in the sense of Ghirardato and Marinacci (2001). Axiom 5 implies that $\succeq^{\prime}$ is convex: if $f^{\prime} \sim g^{\prime}$, then $\frac{1}{2} f^{\prime} \oplus \frac{1}{2} g^{\prime} \succeq^{\prime} f^{\prime}$. This follows almost mechanically from the axiom. Similarly, from Axiom 6 it follows that $f^{\prime} \sim^{\prime} g^{\prime}$ implies $\alpha f^{\prime} \oplus(1-\alpha) x \sim^{\prime} \alpha g^{\prime} \oplus(1-\alpha) x$.

Step 3. Use the results of Ghirardato et al. (2003) to find a general representation for $\succeq^{\prime}$. The previous step proved that the $\succeq^{\prime}$ satisfies all the conditions of Proposition 3 in Ghirardato et al. (2003). This implies that there exists a continuous nonconstant function $u: X \rightarrow \mathbb{R}$ and a nonempty, weak* compact, and convex set $P$ of finitely additive probabilities on the appropriate sigma algebra such that $\succeq^{\prime}$ is represented by

$$
V^{\prime}\left(f^{\prime}\right):=\min _{p \in P} \int_{\Omega^{\prime}} u\left(f^{\prime}\right) \mathrm{d} p .
$$

Step 4. Find a representation of $\succeq$ on constant acts. Define $\Phi^{\prime}$ as a projection of $P$ on $[0,1]$. From the previous step, we obtain a representation of the restriction of $\succeq^{\prime}$ on acts constant on $\Omega$ using the set $\Phi^{\prime}$. We now use this to derive a representation of the restriction of $\succeq$ on $\Delta(X)$, i.e., on constant acts. To do so, we focus on specific mappings from lotteries in $\Delta(X)$ to acts on $[0,1]$ : those that are decreasing, i.e., assign better outcomes to earlier states. Specifically, we say that a mapping $\mu$ from $\Delta(X)$ into the set of measurable functions from $[0,1]$ to $X$ is decreasing and measure-preserving if for all $p \in \Delta(X)$ and all $x \in X, \ell\left([\mu(p)]^{-1}(x)\right)=p(x)$ and $\mu(p)(z) \succeq \mu(p)\left(z^{\prime}\right)$ for $z, z^{\prime} \in[0,1]$ such that $z \leq z^{\prime}$. We say that measure $\phi$ on $[0,1]$ is state-increasing if there exist no $x_{1}, x_{2}, x_{3}$, $x_{4}$ such that $x_{1}<x_{2}<x_{3}<x_{4}, \ell\left(\left[x_{1}, x_{2}\right]\right)=\ell\left(\left[x_{3}, x_{4}\right]\right)$ and $\phi\left(\left[x_{1}, x_{2}\right]\right)>\phi\left(\left[x_{3}, x_{4}\right]\right)$. In other words, $\phi$ assigns a greater weight to equivalent length intervals if they occur at higher values in the state space. Following the representation in Step 3 above and the definition of $\succeq^{\prime}$, it can be then shown that for any decreasing and measure-preserving mapping $\mu$, the restriction of $\succeq$ on $\Delta(X)$ is represented by

$$
U(p):=\min _{\phi \in \Phi} \int_{[0,1]} u(\mu(p)(s)) \mathrm{d} \phi(s),
$$

\footnotetext{
${ }^{29}$ That is, $\mathcal{F}^{\prime}$ is the set of functions $f$ from $\Omega \times[0,1]$ to $X$ measurable with respect to $\Sigma \times \Sigma^{*}$, where $\Sigma$ is the power set of $\Omega$ and $\Sigma^{*}$ is the Borel sigma algebra on $[0,1]$.
} 
where $\Phi$ is the subset of $\Phi^{\prime}$ composed of state-increasing measures. It can be proven that the measures in $\Phi$ are countably additive and atomless, that the Lebesgue measure $\ell$ belongs to $\Phi$, and that all measures in $\Phi$ are mutually absolutely continuous with respect to $\ell$. It can be also shown that $\Phi$ is weak compact.

Step 5. Obtain a multiple prior-multiple distortions representation. Define $\Pi$ as the projection of $P$ on $\Omega$. Note that it is convex and compact. For any $p \in \Delta(X)$, define $c_{p} \in X$ as $\delta_{c_{p}} \sim p$, and for any $f \in \mathcal{F}$, define $f_{c} \in \mathcal{F}$ as $f_{c}(\omega)=\delta_{c_{f(\omega)}}$. By Axiom 2, $f \sim f_{c}$ for all $f$. Combining with Step 3, we obtain

$$
f \succeq g \quad \Leftrightarrow \quad f_{c} \succeq g_{c} \Leftrightarrow \min _{\pi \in \Pi} \int_{\Omega} \pi(\omega) u\left(\delta_{c_{f(\omega)}}\right) \mathrm{d} \omega \geq \min _{\pi \in \Pi} \int_{\Omega} \pi(\omega) u\left(\delta_{c_{g(\omega)}}\right) \mathrm{d} \omega .
$$

Using Axiom 2 and the result in Step 4, we obtain that $\succeq$ is represented by

$$
V(f):=\min _{\pi \in \Pi} \int_{\Omega} \pi(\omega) U(f(\omega)) \mathrm{d} \omega,
$$

where $U$ is defined as in Step 3. We call this representation the multiple priors-multiple distortions representation. Appendix A contains an in-depth discussion.

Step 6. Map to probability weighting. For every $\phi \in \Phi$, construct the corresponding cumulative distribution function, noting that each is convex, strictly decreasing, and differentiable, because every $\phi$ is state-increasing and mutually absolutely continuous with respect to the Lebesgue measure (thus admitting nondecreasing probability density functions that are never flat at zero). Call $\Psi$ the set of cumulative distribution functions (CDFs) obtained. The pointwise compactness of $\Psi$ follows easily. The desired representation then follows.

\subsection{A comparative notion of attraction to certainty}

We now show that the comparative notion of ambiguity aversion introduced in Ghirardato and Marinacci (2002) translates to our setup and implies both more ambiguity aversion and more probability distortions for objective lotteries. Consider two DMs, 1 and 2, such that 2 is more attracted to certainty than 1: whenever 1 prefers a certain option $\delta_{x}$ to some act $f$, so does 2 . This could be interpreted in two ways. First, both agents treat both probabilities and events in the same way, but 2 has a utility function that is more concave than that of 1 . Alternatively, the curvature of the utility function is the same for both agents, but 2 is more pessimistic than 1 is. Ghirardato and Marinacci (2002) focus on this second case-considering the relative attraction to certainty while holding constant the curvature of the utility function. This is obtained by focusing on the case in which $\oplus_{1}=\oplus_{2}$, that is, when both agents have the same approach to outcome mixtures. With this in mind, we use the following definition, introduced by Ghirardato and Marinacci (2002): ${ }^{30}$

\footnotetext{
${ }^{30}$ There are two minor differences between what follows and Definition 7 in Ghirardato and Marinacci (2002). First, we require $\oplus_{\succeq_{1}}=\oplus_{\succeq_{2}}$ instead of requiring that the two preferences are cardinally symmetric, as defined in Definition 5 in Ghirardato and Marinacci (2002). However, these two conditions are equivalent, since both imply that the (unique) utility indexes must be positive affine transformations of each
} 
Definition 5. Let $\succeq_{1}$ and $\succeq_{2}$ be two complete and nondegenerate preference relations on $\mathcal{F}$. We say that $\succeq_{2}$ is more attracted to certainty than $\succeq_{1}$ if the following statements hold:

(i) $\oplus_{\succeq_{1}}=\oplus_{\succeq_{2}}$;

(ii) For all $x \in X$ and all $f \in \mathcal{F}$,

$$
\delta_{x} \succeq_{1} f \Rightarrow \delta_{x} \succeq_{2} f
$$

and

$$
\delta_{x} \succ_{1} f \Rightarrow \delta_{x} \succ_{2} f
$$

for some $x \in X$ and all $f \in \mathcal{F}$.

This has specific consequences in our setup: an agent who is more attracted to certainty must have weakly larger sets of distortions for both objective and subjective uncertainty.

Proposition 1. Let $\succeq_{1}$ and $\succeq_{2}$ be two complete and nondegenerate preference relations on $\mathcal{F}$ that admit minimal multiple priors-multiple weighting representations $\left(u_{1}, \Pi_{1}, \Psi_{1}\right)$ and $\left(u_{2}, \Pi_{2}, \Psi_{2}\right)$. Then the following statements are equivalent:

(i) The relation $\succeq_{2}$ is more attracted to certainty than $\succeq_{1}$.

(ii) The function $u_{1}$ is a positive affine transformation of $u_{2}, \Pi_{2} \supseteq \Pi_{1}$, and $\Psi_{2} \supseteq \Psi_{1}$.

\section{The Ellsberg PARAdox AND Relative Pessimism}

The purpose of this section is to characterize the special case of the MP-MW model in which the behavior of the agent never contradicts the Ellsberg paradox.

To this end, we introduce notation to capture a way to reduce subjective to objective risk. Consider an act $f \in \mathcal{F}$, and suppose that for every state $\omega$ it returns a degenerate lottery, i.e., for all $\omega \in \Omega$, we have $f(\omega)=\delta_{y}$ for some $y \in X$. Consider now a prior $\pi \in \Delta(\Omega)$, and denote by $f^{\pi}$ the lottery in $\Delta(X)$ derived from $f$ using probabilities in $\pi$, that is, the lottery that returns $f(\omega)$ with probability $\pi(\omega)$. This lottery is simply the reduction of $f$ from subjective to objective risk using prior $\pi$. (An identical notion is used in Ok et al. 2012.) We can then also extend this definition to acts that return nondegenerate lotteries, preserving the intuition: for any act $f \in \mathcal{F}$ and prior $\pi \in \Delta(\Omega)$, denote by $f^{\pi}$ the lottery that returns, with probability $\pi(\omega)$, the certainty equivalent of $f(\omega)$. (That is, $f^{\pi}$ denotes the constant act that yields the lottery $\sum \pi(\omega) c_{f(\omega)}$ in every state.)

Endowed with this notation, we can define the following axiom.

other. The second difference is in the name: they interpret it as higher ambiguity aversion, while we interpret it more simply as attraction toward certainty, because here agents could have the same aversion to ambiguity but different pessimism towards objective lotteries. (On this, see Ghirardato and Marinacci 2002 and Ghirardato 2004.) 
Axıом 7 (Preference for objective risk). There exists $\pi \in \Delta(\Omega)$ such that $f^{\pi} \succeq f$ for all $f \in \mathcal{F}$.

The interpretation is straightforward: a DM satisfies preference for objective risk if there is a way to reduce subjective uncertainty to objective risk that would make her (weakly) better off. This postulate is directly related to the intuition of the Ellsberg paradox: the agent would strictly prefer to reduce subjective uncertainty to risk-for example, using the prior that assigns equal probabilities of extracting each color-thus generating an objective lottery rather than keeping the ambiguous Ellsberg bets. In turn, this axiom links the degree of pessimism applied by the agent on objective and subjective bets. Notice that the prior used in Axiom 7 is not related directly to a representation; the axiom only postulates the existence of some probability distribution over $\Omega$ for which the requirement holds.

Our preference for objective risk axiom is naturally linked to the idea of ambiguity aversion. To make this connection more formal, let us begin by defining ambiguity neutrality in our context.

Definition 6. A preference relation $\succeq$ on $\mathcal{F}$ is ambiguity neutral if there exists $\pi \in \Delta(\Omega)$ such that $f^{\pi} \sim f$ for all $f \in \mathcal{F}$.

The notion above is very similar to probabilistic sophistication of Machina and Schmeidler (1995): ${ }^{31}$ the agent reduces subjective to objective risk in a coherent manner, using a probability measure over the states of the world, although she may violate expected utility in evaluating the lottery obtained. In the Ellsberg experiment, an agent who satisfies Definition 6 (with a symmetric prior) would be indifferent between betting on an ambiguous or unambiguous urn. Indeed, the definition above could be considered a way to define, without using independence over objective lotteries, the definition of ambiguity neutrality of Ghirardato and Marinacci (2002).

Endowed with this, we can introduce the following two definitions.

Definition 7. Consider two preference relations $\succeq_{1}$ and $\succeq_{2}$ on $\mathcal{F}$. We say that $\succeq_{2}$ is more ambiguity averse than $\succeq_{1}$ if the following statements hold:

1. The relations $\succeq_{1}$ and $\succeq_{2}$ rank constant acts in the same way, i.e., $p \succeq_{1} q$ if and only if $p \succeq_{2} q$;

2. For all $p \in \Delta(X)$ and $f \in \mathcal{F}$,

$$
p \succeq_{1}\left(\succ_{1}\right) f \Rightarrow p \succeq_{2}\left(\succ_{2}\right) f .
$$

\footnotetext{
${ }^{31}$ The two notions coincide under Axioms 1-3, which implies that preferences that satisfy Definition 6 can be characterized behaviorally following the results in Machina and Schmeidler (1995) (in particular, adding the horse/roulette replacement axiom). At the same time, both notions differ from probabilistic sophistication as defined by Machina and Schmeidler (1992) in a Savage setup, as here we require that not only the agent reduces subjective uncertainty to objective risk using a prior $\pi$, but also that the non-expected utility functional used to evaluate such reduction is the same one used to evaluate objective lotteries.
} 
Definition 8. We say that $\succeq$ is ambiguity averse if there exists a preference relation $\succeq_{\text {an }}$ such that $\succeq_{\text {an }}$ is ambiguity neutral and $\succeq$ is more ambiguity averse than $\succeq_{\text {an }}$.

The definitions above are related to those of Epstein (1999), although we use our notion of ambiguous neutrality instead of probability sophistication (Machina and Schmeidler 1992), use objective lotteries as unambiguous acts, and operate in the setup of Anscombe and Aumann (1963). It is also similar to the definitions of Ghirardato and Marinacci (2002), although the comparison is made with ambiguity-neutral preferences, as opposed to subjective expected utility preferences. ${ }^{32}$

It is straightforward that ambiguity aversion has a natural relationship with our preference for objective risk axiom. This is formalized below.

Proposition 2. Consider a preference relation $\succeq$ on $\mathcal{F}$ that satisfies Axioms 1-3. Then $\succeq$ satisfies Axiom 7 if and only if $\succeq$ is ambiguity averse.

\subsection{Representation theorem}

We now turn to discuss the special case of our representation that excludes the opposite of the Ellsberg paradox. Intuitively, we want a representation that distorts objective bets more than subjective ones. To define this formally, we need to introduce the distortions on priors on $\Omega$ implied by the distortions applied to objective probabilities. To illustrate, suppose that $\Omega=\left\{\omega_{1}, \omega_{2}\right\}, \pi\left(\omega_{1}\right)=\pi\left(\omega_{2}\right)=0.5$, and $\psi(0.5)=0.25$. What are the priors on $\Omega$ obtained by distorting $\pi$ according to $\psi$ ? Because the effects of probability weighting depend on the ranking of alternatives, the distorted prior depends on whether the act that we are evaluating returns the best outcome in state $\omega_{1}$ or $\omega_{2}$. If it is $\omega_{1}$, then we obtain $\hat{\pi}_{1}$ such that $\hat{\pi}_{1}\left(\omega_{1}\right)=\psi\left(\pi\left(\omega_{1}\right)\right)=0.25$ and $\hat{\pi}_{1}\left(\omega_{2}\right)=$ $\psi\left(\pi\left(\omega_{1}\right)+\pi\left(\omega_{2}\right)\right)-\pi\left(\omega_{1}\right)=0.75$. If it is $\omega_{2}$, we obtain $\hat{\pi}_{2}\left(\omega_{1}\right)=0.75$ and $\hat{\pi}_{2}\left(\omega_{2}\right)=0.25$. Thus, both $\hat{\pi}_{1}$ and $\hat{\pi}_{2}$ could be obtained by distorting $\pi$ using $\psi$.

More generally, define by $\mathcal{L}$ the set of all enumerations of $\Omega$, i.e., the set of all bijective maps $l: \Omega \rightarrow\{1, \ldots,|\Omega|\}$. For any probability weighting $\psi$ and enumeration of the states $l \in \mathcal{L}$, define $\gamma_{\psi, l}: \Delta(\Omega) \rightarrow \Delta(\Omega)$ by

$$
\gamma_{\psi, l}(\pi)\left(l^{-1}(1)\right)=\psi\left(\pi\left(l^{-1}(1)\right)\right)
$$

and for all $i=2, \ldots,|\Omega|$,

$$
\gamma_{\psi, l}(\pi)\left(l^{-1}(i)\right)=\psi\left(\sum_{j=1}^{i} \pi\left(l^{-1}(j)\right)\right)-\psi\left(\sum_{j=1}^{i-1} \pi\left(l^{-1}(j)\right)\right) .
$$

It is easy to see that $\gamma_{\psi, l}(\pi)$ is the prior obtained by distorting $\pi$ using probability weighting $\psi$, when the outcomes returned are ordered according to $l$. Using this, we can define

\footnotetext{
${ }^{32}$ The consequences of applying directly the definition of Ghirardato and Marinacci (2002) were discussed in Section 2.5.
} 
the set of all possible distorted priors for a set of probability weightings (as in our representations) and possible enumeration: for any set of probability weightings $\Psi$, define the mapping $\Gamma_{\Psi}: \Delta(\Omega) \rightarrow 2^{\Delta(\Omega)} \backslash\{\varnothing\}$ by

$$
\Gamma_{\Psi}(\pi):=\left\{\gamma_{\psi, l}(\pi): l \in \mathcal{L} \text { and } \psi \in \Psi\right\} .
$$

We are now prepared to introduce our representation theorem for this case.

THEOREM 2. Consider a preference relation $\succeq$ on $\mathcal{F}$ that admits a multiple priorsmultiple weighting representation $(u, \Pi, \Psi)$. The following statements are equivalent:

(i) The relation $\succeq$ satisfies Axiom 7 .

(ii) We have $\Pi \supseteq \Gamma_{\Psi}(\pi)$ for some $\pi \in \Pi$.

Theorem 2 establishes that imposing Axiom 7, preference for objective risk, guarantees that the DM always behaves in line with the Ellsberg paradox. It also shows how the assumption that the DM is ambiguity averse refines our representation, by ensuring that the set of possible distortions due to ambiguity is larger than the set of distortions due to risk. Hedging alone is insufficient to guarantee that this is the case and hence does not guarantee Ellsberg-type behavior.

\section{Overview of the Related literature}

A large literature, considerably too large to be surveyed here, has been devoted to developing models that allow either for Allais or for Ellsberg-type behavior. However, far fewer models exist that permit both features simultaneously in setups in which both phenomena could appear independently, such as that of Anscombe and Aumann (1963). On the one hand, the majority of models designed to study Allais-like behavior focus on objective lotteries only. On the other hand, the vast majority of models designed to capture ambiguity aversion either do not consider objective lotteries, operating in the setup of Savage (1954), or do consider them, operating in the setup of Anscombe and Aumann (1963), but assume that agents satisfy vNM independence on objective lotteries. ${ }^{33}$

From the point of view of the literature on ambiguity aversion, our paper can be considered a generalization of MMEU that allows for Allais-type preferences over objective lotteries. Indeed, ours is not the first paper to generalize MMEU in this direction. Ghirardato et al. $(2001,2003)$ show that one can obtain an MMEU-like representation on Savage acts by considering outcome mixtures and disregarding objective lotteries, thus not restricting, but also not modeling, how the agent reacts to them. Drapeau and Kupper (2013) introduce a model that corresponds to one in which agents exhibit uncertainty-averse preferences as in Cerreia-Vioglio et al. (2011b) on acts that do not

\footnotetext{
${ }^{33}$ This is imposed through an axiom called risk independence, which is vNM independence for objective lotteries, and is implied by most weakenings of independence suggested in this literature (e.g., comonotonic independence, certainty independence, weak certainty independence). It is also imposed in the much more general models of Cerreia-Vioglio et al. (2011a, 2011b) and Ghirardato and Siniscalchi (2012). (See Gilboa and Marinacci 2016 for a survey.)
} 
involve objective lotteries and react to objective risk in a way similar to the model in Cerreia-Vioglio (2009). A corollary to the main theorem in Klibanoff et al. (2005) generalizes their representation to the case of non-EU preferences on objective lotteries; this case, however, is not fully axiomatized and does not model jointly attitudes toward risk and uncertainty. ${ }^{34}$

From the point of view of the literature on Allais-like behavior, we have already argued (Section 2.3) that our model is a generalization of RDU with convex distortions and is therefore related to models of probability weighting. Safra and Segal (1998) derive a model (Lemma 4) that similarly generalizes RDU for the case of linear utility, beginning from an axiom, diversification, that in case of linear utilities is related to our hedging. In addition, our model is conceptually related to models in which the agent does not distort probabilities but has a set of utility functions and uses the most pessimistic one of them to evaluate a lottery. These models include Maccheroni (2002) and the cautious expected utility model of Cerreia-Vioglio et al. (2015), both of which are special cases of the model in Cerreia-Vioglio (2009). While neither studies ambiguity aversion, instead focusing on risk, these models are conceptually related to ours-in some sense, they are their counterpart: while in our case the agent has a fixed utility function and multiple probabilities, in these models the agent uses the correct probabilities but has multiple utility functions. In Maccheroni (2002), this conceptual difference entails also a difference in behavior, as this model need not be compatible with Allais-like behavior. The cautious expected utility model of CerreiaVioglio et al. (2015) is instead designed to model Allais-like behavior and is characterized by one axiom that captures it: negative certainty independence (NCI, introduced by Dillenberger 2010). Interestingly, while Cerreia-Vioglio et al. (2015) prove that the only intersection between the cautious expected utility model and RDU is expected utility, there exist examples of preferences that can be represented as the minimum of convex RDU, as in our model, that also admit a cautious expected utility representation and yet are not expected utility. ${ }^{35}$ Thus, on the one hand, our axioms allow for behavior that is ruled out by NCI (e.g., RDU with strictly convex distortions), but on the other hand, the generalization of RDU characterized in this paper is compatible with both NCI and the violation of expected utility. The model in this paper allows for preferences that can be strictly convex, but also the opposite, or satisfy betweenness: by contrast, under cautious expected utility, preferences must be weakly convex, while in pessimistic RDU, they must be the opposite. ${ }^{36}$ The exact

\footnotetext{
${ }^{34}$ In addition, Chew and Sagi (2008) suggest how using the notion of "conditional small worlds" that they introduce could generate a behavior that is consistent with both the Ellsberg and the Allais paradoxes. Abdellaoui et al. (2011) also consider a general setup in which DMs are non-expected utility maximizers within the same source of uncertainty (which could be objective), but may have different weighting functions for different sources, generating ambiguity aversion.

${ }^{35}$ These examples are available from the authors upon request.

${ }^{36}$ The experimental evidence on convexity of preferences is mixed: while papers that study violations of betweenness document deviations in both directions (that is, either preference or aversion to mixing), Sopher and Narramore (2000), Dwenger et al. (2015), and Agranov and Ortoleva (2017) find explicit evidence in support of convexity. Models that allow for strictly convex preferences can be used to study random choice as a deliberate randomization, as in Machina (1985) and Cerreia-Vioglio et al. (2017).
} 
relationship between NCI and our hedging axiom (Axiom 5) remains an open question.

Our paper uses outcomes instead of probability mixtures. Procedures of this type were introduced by Gul (1992) and appear in Chew and Karni (1994), Wakker (1994), Casadesus-Masanell et al. (2000a, 2000b), Ghirardato et al. (2001, 2003), and Köbberling and Wakker (2003). Our approach can be regarded as the translation of that of Ghirardato et al. (2003) to the case of objective probabilities. ${ }^{37}$ We then use this approach to introduce the novel notion of an outcome mixture of lotteries and of acts, a central step in our analysis.

An early approach to relating violations of expected utility observed in the objective and subjective domains came from noting that the RDU representation is identical to the Choquet expected utility model of ambiguity of Schmeidler (1989) (see, for example, Wakker 2001). In contrast to this literature, we study the case of the simultaneous presence of ambiguity aversion and Allais-like behavior instead of focusing on one of them. ${ }^{38}$ Wakker (2001) is closely related to our contribution and focuses on the case in which the preferences of the agent are of the Choquet expected utility form for both risk and ambiguity, and shows that a generalization of the common consequence effect can be used to characterize pessimism in both the objective and subjective domains. Our generalized preference for hedging could be seen as an assumption with a similar spirit, although applicable to a broader class of preferences (MMEU is more general than pessimistic Choquet expected utility, and we have shown that our representation generalizes convex RDU). However, to define our notion, we use outcome mixtures, which forces us to impose a richer structure on the space of consequences (connectedness). Wakker (2001) also shows that the conditions that imply pessimism for subjective uncertainty do not guarantee Ellsberg-type behavior in the presence of non-EU behavior over objective risk. We obtain a similar result and include a novel axiom that allows us to characterize a model in which this is guaranteed.

Segal $(1987 a, 1990)$ suggests a different channel to connect the Allais and Ellsberg paradoxes: both can be interpreted as stemming from a failure to reduce compound lotteries. In particular, Segal (1990) shows how RDU can be derived from such a postulate, and Segal (1987a) argues that the Ellsberg paradox can be seen in a similar light if an ambiguous lottery is treated as a two-stage lottery, where the first, imaginary, stage

\footnotetext{
${ }^{37}$ The difference is that we construct subjective mixtures using objective lotteries, and all we need is biseparability of preferences over 50/50 lotteries-a weak requirement, as the vast majority of models of nonEU under risk are bi-separable. By contrast, in Ghirardato et al. (2003) objective lotteries are not studied, and they require local bi-separability in preferences over acts. This could be a demanding requirement: for example, many models of preference under uncertainty do not satisfy it (e.g., the variational preferences of Maccheroni et al. 2006).

${ }^{38}$ This is possible because we use the standard setup of Anscombe and Aumann (1963), where both features could be present at the same time and independently. By contrast, the approach followed by most of the literature would not apply in this setup, and, in general, would not apply when lotteries are elements of the simplex, as in von Neumann and Morgenstern or as in the questions of the Allais experiment: in most of these papers, lotteries are Savage acts on a given set of states of the world with an objective probability distribution.
} 
is over the possible priors. This connection, however, is based on a specific interpretation of the Ellsberg paradox and on the richer setup in which two-stage lotteries are observable. $^{39}$

As discussed above, our paper is also related to Gumen et al. (2014), who analyze subjective distortions of objective probabilities and define a behavioral notion of pessimism for risky prospects that is very reminiscent of uncertainty aversion. The connection with our work is twofold. First, as we mentioned above, for bi-separable preferences, our hedging axiom coincides with the notion of pessimism they introduce. Second, in Appendix A, we offer an alternative but equivalent representation in which objective lotteries are treated as ambiguous objects, a model conceptually related to their subjective risk. The two papers are very different, however, as their goal is to introduce a generalized notion of pessimism, not to provide a characterization theorem.

\section{APPENDix A: AN ALternative REPRESENTATION OBJECTIVE LOTTERIES AS} AMBIGUOUS OBJECTS

We now introduce an alternative representation, equivalent to the main one as well as to the axioms, that provides a different interpretation: in this case, the pessimism of the agent comes from the fact that the DM treats objective lotteries as "ambiguous objects."

In this representation, it is as if the agent imagined that an urn of measure 1 were used to determine the prize of each objective lottery, as follows: for any lottery $p$, each ball in this imaginary urn is assigned a consequence in $X$, starting from the worst prizes of $p$ and proceeding to better ones. This creates an act that maps from the state space $[0,1]$ to $X$. Such acts must be constructed in a way that is coherent with the original lottery: the Lebesgue measure of balls that give $x$ as a prize must be the same as the probability of obtaining $x$ from $p$. The prize from the lottery is then determined by extracting a ball from this urn. Formally, we define a map from objective lotteries into acts on $[0,1]$ that assigns the correct number of balls to each prize, depending on the original lottery and such that the worst prizes are assigned to smaller states.

Definition 9. We say that a map $\mu$ from $\Delta(X)$ to the set of measurable functions from $[0,1]$ to $X$ is decreasing and measure-preserving if for all $p \in \Delta(X)$ and all $x \in X$, $\ell\left([\mu(p)]^{-1}(x)\right)=p(x)$ and $\mu(p)(z) \succeq \mu(p)\left(z^{\prime}\right)$ for $z, z^{\prime} \in[0,1]$ such that $z \leq z^{\prime}{ }^{40}$

To evaluate these acts, an expected utility maximizer would use the Lebesgue measure as a prior over the balls in the urn. An agent who departs from expected utility because she is pessimistic could instead use a different criterion, as in the following representation.

\footnotetext{
${ }^{39}$ One could also see the setup of Anscombe and Aumann (1963) as "rich," as it entails both objective and subjective uncertainty with an implicit assumption about the timing of the resolution of each of them. As we argued in Section 2.1, however, this feature is irrelevant for us: we could have carried out our analysis even if we only observed the agent's preference over the union of vNM lotteries and of Savage acts.

${ }^{40}$ In practice, such a mapping can be constructed for any lottery $p$ as follows: (i) enumerate the outcomes in $\operatorname{supp}(p)$ from best to worst, i.e., $x_{i-1} \succeq x_{i}$ for $i=2, \ldots,|\operatorname{supp}(p)|$; (ii) define $\mu(p)$ as $\mu(p)\left(\left[0, p\left(x_{1}\right)\right)\right)=x_{1}$ and $\mu(p)\left(\left[\sum_{j=1}^{i-1} p\left(x_{i}\right), \sum_{j=1}^{i} p\left(x_{i}\right)\right)\right)=x_{i}$ for $i=2, \ldots,|\operatorname{supp}(p)|$.
} 
Definition 10. Consider a complete and nondegenerate preference relation $\succeq$ on $\mathcal{F}$. We say that $\succeq$ admits a multiple priors and multiple distortions representation (MP-MD) $(u, \Pi, \Phi)$ if there exists a continuous utility function $u: X \rightarrow \mathbb{R}$, a convex and compact set of probability measures $\Pi$ on $\Omega$, and a convex and weak-compact set of Borel probability measures $\Phi$ on $[0,1]$, which contains the Lebesgue measure $\ell$ and such that every $\phi \in \Phi$ is atomless, state increasing, ${ }^{41}$ and mutually absolutely continuous with respect to $\ell$, such that $\succeq$ is represented by the functional

$$
V(f):=\min _{\pi \in \Pi} \int_{\Omega} \pi(\omega) U(f(\omega)) \mathrm{d} \omega,
$$

where $U: \Delta(X) \rightarrow \mathbb{R}$ is defined as

$$
U(p):=\min _{\phi \in \Phi} \int_{[0,1]} u(\mu(p)(s)) \mathrm{d} \phi(s)
$$

for any decreasing and measure-preserving map $\mu$ from $\Delta(X)$ to the set of measurable functions from $[0,1]$ to $X$.

In this representation, it is as if the DM was treating objective lotteries like ambiguous acts. First, she "converts" a lottery into an act on [0,1] (in a decreasing and measure-preserving fashion). Then she evaluates it using the most pessimistic of a set of beliefs-exactly as she evaluates other acts: she uses the worst of a set of priors $\Phi$ over $[0,1]$. When $\Phi=\{\ell\}$, her evaluation of lotteries is equivalent to vNM expected utility. But when $\Phi \supset\{\ell\}$, her ranking of objective lotteries will be pessimistic, (weakly) lowering their evaluation by using a prior in $\Phi$ that gives a lower value than $\ell$. It is as if she were thinking that states (balls in the urn) associated with better outcomes were less likely than those associated with bad ones. Since her valuation of degenerate lotteries will not be affected—as it is independent from the prior in $\Phi$ that it is used-this leads to certainty bias and Allais-like behavior.

THEOREм 3. Consider a complete and nondegenerate preference relation $\succeq$ on $\mathcal{F}$. Then the following statements are equivalent:

(i) The relation $\succeq$ satisfies Axioms 1-6.

(ii) There exists a multiple priors-multiple weighting representation $(u, \Pi, \Psi)$ of $\succeq$.

(iii) There exists a multiple priors and multiple distortions representation $(u, \Pi, \Phi)$ of $\succeq$.

\section{Appendix B: Relation with the Allais paradox}

In Section 2.3 we have argued how the MP-MW can allow for the behavior observed in the common consequence effect. Here we show that the model rules out violations of

\footnotetext{
${ }^{41} \mathrm{~A}$ prior $\phi$ on $[0,1]$ is state-increasing if there do not exist any $x_{1}, x_{2}, x_{3}, x_{4}$ such that $x_{1}<x_{2}<x_{3}<x_{4}$, $\ell\left(\left[x_{1}, x_{2}\right]\right)=\ell\left(\left[x_{3}, x_{4}\right]\right)$, and $\phi\left(\left[x_{1}, x_{2}\right]\right)>\phi\left(\left[x_{3}, x_{4}\right]\right)$.
} 
expected utility that go in the opposite direction. To wit, consider the following four lotteries: $p_{1}=\$ z, p_{2}=r \cdot \$ 0+q \cdot \$ z+(1-r-q) \cdot \$ x, p_{3}=q \cdot \$ 0+(1-q) \cdot \$ z$, and $p_{4}=(r+q) \cdot \$ 0+(1-r-q) \$ y$ for $r+q<1$. Recall that the Allais experiment asked subjects to compare the lotteries above assuming $x=y=\$ 5,000,000, z=\$ 1,000,000, r=$ 0.01 , and $q=0.89$, and then observed the first lottery preferred to the second, but the fourth preferred to the third. Let us now instead choose $x$ and $y$ in such a way to make $p_{1} \sim p_{2}$ and $p_{3} \sim p_{4}$. Then we have a choice pattern that conforms with Allais if and only if $x \geq y$.

So as to show that the MP-MW model implies that this must be the case, we make use of a variant of the MP-MD model that we show in Appendix A to be equivalent. For simplicity, we relax the necessity that the measure-preserving maps have to be decreasing. Let $(u, \Pi, \Phi)$ be such an MP-MD representation. Normalize $u(z)=1$ and $u(0)=0$. We show that $u(x)$ must be greater than or equal to $u(y)$ and so $x \geq y$. Let us define the following three events on the unit interval: $E_{1}=[0, q), E_{2}=[q, q+r), E_{3}=(q+r, 1]$. Then consider the (measure-preserving) map from lotteries into acts on $[0,1]$ defined by the table

$\begin{array}{llll} & E_{1} & E_{2} & E_{3} \\ p_{1} & \$ z & \$ z & \$ z \\ p_{2} & \$ z & \$ 0 & \$ x \\ p_{3} & \$ 0 & \$ z & \$ z \\ p_{4} & \$ 0 & \$ 0 & \$ y\end{array}$

Let $\alpha$ be the smallest weight put on $E_{3}$ by any prior in $\Phi$, and let $\beta$ be the smallest weight put on $E_{2}$ by one of the priors for which $\phi\left(E_{2}\right)=\alpha$. Notice first of all that we must have $U\left(p_{2}\right) \leq(1-\alpha-\beta)+\alpha u(x)$, since $p_{2}$ could be evaluated using the prior above or a worse one, so $1=U\left(p_{1}\right)=U\left(p_{2}\right) \leq(1-\alpha-\beta)+\alpha u(x)$; hence, $(\alpha+\beta) / \alpha \leq u(x)$. Notice also that we must have $U\left(p_{4}\right)=\alpha u(y)$ and $U\left(p_{3}\right) \leq \min (1-q, \alpha+\beta) .{ }^{42}$ Suppose first that we have $\alpha+\beta \leq 1-q$. Then $\alpha u(y)=U\left(p_{4}\right)=U\left(p_{3}\right) \leq \alpha+\beta$; hence, $u(y) \leq(\alpha+\beta) / \alpha$, which means $x \geq y$ as desired. Suppose instead that $\alpha+\beta>1-q$. This means that we have $\alpha u(y)=U\left(p_{4}\right)=U\left(p_{3}\right) \leq 1-q$, so $u(y) \leq(1-q) / \alpha$. Since $u(x) \geq(\alpha+\beta) / \alpha$ and $\alpha+\beta>1-q$ we have $u(x)>(1-q) / \alpha$, so $x>y$. Thus $x \geq y$ as sought.

\section{Appendix C: Hedging neutrality and Restricted violations}

The main representation in this paper allows for the simultaneous violations of both Anscombe-Aumann expected utility on acts and vNM expected utility on objective lotteries. We now turn to analyze the behavioral axioms that allow us to restrict violations to only one of these domains, which amounts to different forms of hedging neutrality.

Axıом 8 (Hedging neutrality). For any $f, g \in \Delta(X)$ and for any $h \in f \oplus g$, if $f \sim g$, then $h \sim f$.

Axıом 9 (Hedging neutrality on acts). For any $f, g, h \in \mathcal{F}$ such that $f \sim g, h \in f \oplus g$ and such that for all $\omega \in \Omega$ we have $f(\omega)=\delta_{x}$ and $g(\omega)=\delta_{y}$ for some $x, y \in X$, we have $h \sim f$.

${ }^{42}$ We know that $u\left(p_{3}\right) \leq 0.11$ since $\Phi$ contains the Lebesgue measure. 
Aхıом 10 (Hedging neutrality on lotteries). For any $p, q, r \in \Delta(X)$ such that $p \sim q$ and $r \in p \oplus q$, we have $r \sim p$.

A different way to capture hedging neutrality is to posit that the agent is indifferent between subjective and objective mixtures. The following axiom imposes this in a very weak form: that there exists at least one situation in which probability and outcome mixtures coincide.

Axıом 11 (Local neutrality for subjective and objective mixtures). There exists $x, y \in X$ and a dyadic $\lambda \in(0,1)$ such that $\delta_{x} \nsim \delta_{y}$ and $\lambda x+(1-\lambda) y \sim \delta_{\lambda x \oplus(1-\lambda) y}$.

The following proposition shows the effects of including these axioms in our framework.

Proposition 3. Consider a nondegenerate preference relation $\succeq$ that admits a multiple priors-multiple weighting representation $(u, \Pi, \Psi)$. The following hold:

(a) We have $|\Pi|=1$ if and only if $\succeq$ satisfies Axiom 9 (hedging neutrality on acts).

(b) The following statements are equivalent:

(i) The set $\Psi$ contains only the identity function.

(ii) The relation $\succeq$ satisfies Axiom 10 (hedging neutrality on lotteries).

(iii) The relation $\succeq$ satisfies independence restricted to $\Delta(X)$.

(iv) The relation $\succeq$ satisfies Axiom 11 (local neutrality for subjective and objective mixtures).

(c) The following statements are equivalent:

(i) We have $|\Pi|=|\Psi|=1$, and $\Psi$ contains only the identity function.

(ii) The relation $\succeq$ satisfies Axiom 8 (hedging neutrality).

(iii) The relation $\succeq$ satisfies independence.

We emphasize point (b) in particular: under the axiomatic structure of our representation, we obtain expected utility on objective lotteries simply by imposing Axiom 11, i.e., that there exists at least one nontrivial case in which subjective and objective mixtures coincide.

Proof of Proposition 3. The proof of all the steps except the equivalence between (b) (i) and (b) (iv) follows standard arguments and is therefore omitted. If $\Psi$ contains only the identity function, it is also trivial to see that Axiom 11 is satisfied. Assume now that Axiom 11 holds and that $\succeq$ admits a representation $(u, \Pi, \Psi)$. We now argue that we must have $|\Psi|=1$ and that it contains only the identify function. Suppose this is not the case and say $\psi \in \Psi$, where $\psi$ is not the identity function. Since $\psi$ must be convex and it 
must be a probability weighting function (increasing, $\psi(0)=0, \psi(1)=1$ ), then we must also have that $\psi(x)>x$ for all $x \in(0,1)$. But this implies that we have $\operatorname{RDU}_{u, \psi}(\alpha x+(1-$ $\alpha) y)<\alpha u(x)+(1-\alpha) u(y)$. Since $\psi \in \Psi$, we must then have that for all $x, y \in X$ such that $u(x) \neq u(y)$, and for all $\alpha \in(0,1)$, we must have $\alpha x+(1-\alpha) y \prec \delta_{\alpha x \oplus(1-\alpha) y}$, violating Axiom 11.

\section{Appendix D: Proofs}

For the purpose of this section, let us add the following notation: given some $p, q \in$ $\Delta(X)$ and some $A \subseteq \Omega, p E q$ denotes the acts that yield lottery $p$ if $E$ is realized, and $q$ otherwise.

Proof of Theorems 1 and 3. Since Theorem 1 is a special case of Theorem 3, it will suffice to prove the latter.

(1) $\Rightarrow$ (3). The proof will proceed with the following seven steps: (i) we construct a derived preference relation on the Savage space with consequences $X$ and set of states $\Omega \times[0,1]$; (ii) we prove that the continuity properties of the original preference relation imply some continuity properties of the derived preference relation; (iii) we prove that this derived relation is locally bi-separable (in the sense of Ghirardato and Marinacci 2001) for some event in the space $\Omega \times[0,1]$; (iv) we prove that the axioms imposed on the original preference imply that the derived preference satisfies the axioms required by Ghirardato et al. (2003) that, in turn, implies the existence of a representation similar to maxmin expected utility, but in the larger Savage space; (v) we use this result to provide a representation for the restriction of $\succeq$ to constant acts; (vi) using monotonicity, we obtain the desired representation; (vii) we show that we can focus only on decreasing measure-preserving maps and state-increasing priors over [0,1].

Step 1. Denote by $\Sigma$ the power set of $\Omega$, denote by $\Sigma^{*}$ the Borel $\sigma$-algebra on $[0,1]$, and consider a state space $\Omega^{\prime}:=\Omega \times[0,1]$ with the appropriate sigma algebra $\Sigma^{\prime}:=\Sigma \times \Sigma^{*}$. Define $\mathcal{F}^{\prime}$ to be the set of simple Savage acts on $\Omega^{\prime}$, i.e., $\Sigma^{\prime}$-measurable, finite valued functions $f^{\prime}: \Omega^{\prime} \rightarrow X$. To avoid confusion, we use $f^{\prime}, g^{\prime}, \ldots$ to denote generic elements of this space. ${ }^{43}$ Define $\oplus$ on $\mathcal{F}^{\prime}$ like we did in $\mathcal{F}$ : once we have $\oplus$ defined on $X$, for any $f^{\prime}, g^{\prime} \in \mathcal{F}^{\prime}$ and $\alpha \in(0,1), \alpha f^{\prime} \oplus(1-\alpha) g^{\prime}$ is the act in $\mathcal{F}^{\prime}$ such that $\left(\alpha f^{\prime} \oplus(1-\alpha) g^{\prime}\right)\left(\omega^{\prime}\right)=\alpha f^{\prime}\left(\omega^{\prime}\right) \oplus(1-\alpha) g^{\prime}\left(\omega^{\prime}\right)$ for all $\omega^{\prime} \in \Omega^{\prime}$. (Moreover, since each act in $\mathcal{F}^{\prime}$ is a function from $\Omega \times[0,1]$ into $X$, for all $f^{\prime} \in \mathcal{F}^{\prime}$ and for all $\omega \in \Omega$, abusing notation, we can denote $f^{\prime}(\omega, \cdot):[0,1] \rightarrow X$ as the act that is constant in the first component $(\Omega)$ but not on the second component $([0,1])$.)

We now define two maps, one from $\mathcal{F}$ to $\mathcal{F}^{\prime}$ and the other from $\mathcal{F}^{\prime}$ to $\mathcal{F}$. Define first of all $\gamma^{-1}: \mathcal{F}^{\prime} \rightarrow \mathcal{F}$ as

$$
\gamma^{-1}\left(f^{\prime}\right)(\omega)(x)=\ell\left(f^{\prime}(\omega, \cdot)^{-1}(x)\right),
$$

where $\ell(\cdot)$ denotes the Lebesgue measure. It is easy to see that $\gamma^{-} 1(f)$ is well defined. Now define $\gamma: \mathcal{F} \rightarrow 2^{\mathcal{F}^{\prime}}$ as

$$
\gamma(f)=\left\{f^{\prime} \in \mathcal{F}^{\prime}: f=\gamma^{-1}\left(f^{\prime}\right)\right\} .
$$

\footnotetext{
${ }^{43}$ Following the same abuses of notation in the main setup, for any $x \in X$, we also refer to the constant act $x \in \mathcal{F}^{\prime}$, which returns $x$ in every state.
} 
Notice that, by construction, we must have $\gamma(f) \cap \gamma(g)=\varnothing$ for all $f, g \in \mathcal{F}$ such that $f \neq g$. (Otherwise, we would have some $f^{\prime} \in \mathcal{F}^{\prime}$ such that $\gamma^{-1}\left(f^{\prime}\right)=f$ and $\gamma^{-1}\left(f^{\prime}\right)=g$, which is not possible since $f \neq g$.) Moreover, notice that we must have that $\gamma\left(\delta_{x}\right)=\{x\}$. Finally, notice that $\gamma \mathcal{F}:=\bigcup_{f \in \mathcal{F}} \gamma(f)=\mathcal{F}^{\prime}$ by construction.

Define now $\succeq^{\prime}$ on $\mathcal{F}^{\prime}$ as follows: $f^{\prime} \succeq^{\prime} g^{\prime}$ if and only if $f \succeq g$ for some $f, g \in \mathcal{F}$ such that $f=\gamma^{-1}\left(f^{\prime}\right)$ and $g=\gamma^{-1}\left(g^{\prime}\right)$. Define by $\sim^{\prime}$ and $\succ^{\prime}$ the symmetric and asymmetric parts. (Notice that this implies $f^{\prime} \sim^{\prime} g^{\prime}$ if $f^{\prime}, g^{\prime} \in \gamma(f)$ for some $f \in \mathcal{F}$.)

Note that $\succeq^{\prime}$ is a complete preference relation on $\mathcal{F}^{\prime}$. The completeness of $\succeq^{\prime}$ is a trivial consequence of the completeness of $\succeq$ and the fact that $\gamma(\mathcal{F})=\mathcal{F}^{\prime}$. Similarly, the reflexivity follows from the reflexivity of $\succeq^{\prime}$. To prove that $\succeq^{\prime}$ is transitive, consider some $f^{\prime}, g^{\prime}, h^{\prime} \in \mathcal{F}^{\prime}$ such that $f^{\prime} \succeq^{\prime} g^{\prime}$ and $g^{\prime} \succeq^{\prime} h^{\prime}$. By construction, we must have some $f, g, h \in \mathcal{F}$ such that $f=\gamma^{-1}\left(f^{\prime}\right), g=\gamma^{-1}\left(g^{\prime}\right)$, and $h=\gamma^{-1}\left(h^{\prime}\right)$ such that $f \succeq g$ and $g \succeq h$. By transitivity of $\succeq$, we also have $f \succeq h$; hence, $f^{\prime} \succeq^{\prime} h^{\prime}$ as sought.

Step 2. We now prove that the continuity properties of $\succeq$ are inherited by $\succeq^{*}$. In particular, we prove that for any $\left(f_{n}\right) \in(\mathcal{F})^{\infty}, f \in \mathcal{F}$, if there exists $\left(f_{n}^{\prime}\right) \in\left(\mathcal{F}^{\prime}\right)^{\infty}, f^{\prime} \in \mathcal{F}^{\prime}$ such that $f_{n}=\gamma^{-1}\left(f_{n}^{\prime}\right)$ for all $n, f=\gamma^{-1}\left(f^{\prime}\right)$, and such that $f_{n}^{\prime} \rightarrow f^{\prime}$ pointwise, then we must have that $f_{n} \rightarrow f$.

To prove this, we will focus on the case in which $f_{n}$ and $f$ are constant acts, i.e., $f_{n}, f \in \Delta(X)$. The extension to the general case follows trivially. Assume that $f_{n}^{\prime}$ and $f^{\prime}$ as above exist: we will now prove that if $p_{n}=\gamma^{-1}\left(f_{n}^{\prime}\right)$ for all $n$ and if $p=\gamma^{-1}(f)$, then $p_{n} \rightarrow p$ (weakly). Consider now some continuous $v$ and notice that we must have that $\int_{X} v(u) \mathrm{d} p_{n}=\int_{[0,1]} v\left(f_{n}^{\prime}\right) \mathrm{d} \ell$ by construction of $\gamma$. (Recall that $\ell$ is the Lebesgue measure.) Moreover, since $v$ is continuous and since $f_{n}^{\prime}$ pointwise converges to $f^{\prime}$, we must then have that $\int_{[0,1]} v\left(f_{n}^{\prime}\right) \mathrm{d} \ell \rightarrow \int_{[0,1]} v\left(f^{\prime}\right) \mathrm{d} \ell=\int_{X} v(u) \mathrm{d} p$ : in turn, this means $\int_{X} v(u) \mathrm{d} p_{n}=$ $\int_{X} v(u) \mathrm{d} p$. Since this was proved for a generic continuous $v$, we must have $p_{n} \rightarrow p$ (in weak convergence).

Step 3. We now prove that $\succeq^{\prime}$ is locally bi-separable for some event $A \in \Sigma^{\prime}$. Consider the event $A=\Omega \times\left[0, \frac{1}{2}\right]$. Define $\Sigma_{A}$ as the algebra generated by $A$, i.e., $\Sigma_{A}:=$ $\left\{\varnothing, A, A^{C}, \Omega^{\prime}\right\}$, and by $\mathcal{F}_{A}^{\prime}$ the corresponding set of acts, which is a subset of $\mathcal{F}^{\prime}$. We will now prove that the restriction of $\succeq^{\prime}$ on acts measurable under $\Sigma_{A}$ is bi-separable in the sense of Ghirardato and Marinacci (2001).

Note first that there exist $x, y \in X$ such that $\delta_{x} \succ \delta_{y}$. To see why, suppose, by means of contradiction, that $\delta_{x} \sim \delta_{y}$ for all $x, y \in X$. Then we would have that $p \unrhd_{\mathrm{FOSD}} q$ for all $p, q \in \Delta(X)$. By Axiom 1 (FOSD), therefore, we would have $p \sim q$ for all $p, q \in \Delta(X)$. In turn, by Axiom 2 (monotonicity) we must have $f \sim g$ for all $f, g \in \mathcal{F}$, but this contradicts the assumption that $\succeq$ is nondegenerate.

Notice also that for every $f^{\prime}, g^{\prime} \in \mathcal{F}^{\prime}$, if $f^{\prime}\left(\omega^{\prime}\right) \succeq^{\prime} g^{\prime}\left(\omega^{\prime}\right)$ for every $\omega^{\prime} \in \Omega^{\prime}$, then $f^{\prime} \succeq g^{\prime}$. To see why, consider some $f^{\prime}, g^{\prime} \in \mathcal{F}^{\prime}$ such that $f^{\prime}\left(\omega^{\prime}\right) \succeq^{\prime} g^{\prime}\left(\omega^{\prime}\right)$ for every $\omega^{\prime} \in \Omega^{\prime}$. Now consider $f^{\prime}(\omega, \cdot)$ and $g^{\prime}(\omega, \cdot)$ for some $\omega \in \Omega$, and notice that we have that both $\gamma^{-1}\left(f^{\prime}(\omega, \cdot)\right)$ and $\gamma^{-1}\left(g^{\prime}(\omega, \cdot)\right)$ are constant acts (in $\left.\mathcal{F}\right)$. Since we have $f^{\prime}(\omega, A) \succeq^{\prime}$ $g^{\prime}(\omega, A)$ for all $A \in \Sigma^{*}$, and since $x \succeq^{\prime} y$ if and only if $\delta_{x} \succeq \delta_{y}$, then we must also have that $\gamma^{-1}\left(f^{\prime}(\omega, \cdot)\right) \unrhd_{\text {FOSD }} \gamma^{-1}\left(g^{\prime}(\omega, \cdot)\right)$ by construction. By Axiom 1 (FOSD) then, we must have $\gamma^{-1}\left(f^{\prime}(\omega, \cdot)\right) \succeq \gamma^{-1}\left(g^{\prime}(\omega, \cdot)\right)$ for all $\omega \in \Omega$. In turn, this means that, for the acts $\hat{f}, \hat{g} \in \mathcal{F}$ defined by $\hat{f}(\omega):=\gamma^{-1}\left(f^{\prime}(\omega, \cdot)\right)$ and $\hat{g}(\omega):=\gamma^{-1}\left(g^{\prime}(\omega, \cdot)\right)$ for all $\omega \in \Omega$, we have 
$\hat{f} \succeq \hat{g}$ by Axiom 2 (monotonicity). But then notice that we must have that $f^{\prime} \in \gamma(\hat{f})$ and $g^{\prime} \in \gamma(\hat{g})$ by construction. But this means that we have $f^{\prime} \succeq^{\prime} g^{\prime}$ as sought.

Claim 1. For any $x, y \in X, \gamma^{-1}(x A y)=\frac{1}{2} x+\frac{1}{2} y$.

Proof. Notice first of all that since $x A y \in \mathcal{F}^{\prime}$ is a constant act, then so must be $\gamma^{-1}(x A y)$. Moreover, notice that by definition of $\gamma^{-1}$ we must have that for all $\omega \in \Omega$, $\gamma^{-1}(x A y)(\omega)(x)=\frac{1}{2}$; similarly, for all $\omega \in \Omega, \gamma^{-1}(x A y)(\omega)(y)=\frac{1}{2}$. This implies that we have $\gamma^{-1}(x A y)(\omega)(x)=\frac{1}{2} x+\frac{1}{2} y$ as sought.

Now notice that for every $x, y \in X$, there exists $z \in X$ such that $z \sim^{\prime} x A y$. To see why, consider $x, y \in X$, and notice that $\gamma^{-1}(x A y)=\frac{1}{2} x+\frac{1}{2} y$ by Claim 1 . Now notice that by Axiom 3 (continuity) and Axiom 1 (FOSD), there must exist $z \in X$ such that $\frac{1}{2} x+\frac{1}{2} y \sim \delta_{z}$. We have previously observed that $\gamma^{-1}(z)=\delta_{z}$, which implies $\gamma^{-1}(z) \sim \gamma^{-1}(x A y)$, which implies $x A y \sim^{\prime} z$ as sought.

Given the above discussion, for any $x, y \in X$, define $c e^{\prime}(x A y):=z$ for some $z \in X$ such that $x A y \sim^{\prime} z$. Now notice that $A$ is an essential event for $\succeq^{\prime}{ }^{44}$ To see why, consider any $x, y \in X$ such that $\delta_{x} \succ \delta_{y}$ (note that we have proved that they exist). Now consider the $p=\frac{1}{2} x+\frac{1}{2} y$. By Axiom 1 (FOSD) we must have $\delta_{x} \succ p \succ \delta_{y}$. Now consider the act $x A y \in \mathcal{F}^{\prime}$. Notice that we have $x A y(\omega \times[0,5])=x$ and $x A y(\omega \times[0.5,1])=\delta_{y}$ for all $\omega \in \Omega$. By construction, therefore, we must have $x A y \in \gamma(p), x \in \gamma\left(\delta_{x}\right)$ and $y \in \gamma\left(\delta_{y}\right)$. By definition of $\succ^{\prime}$, then we have $x \succ^{\prime} x A y \succ^{\prime} y$ as sought.

Claim 2 (A Monotonicity). For any nonnull event $B \in \Sigma_{A}$ and $x, y, z \in X$ such that $x, y \succ$ $z$ we have

$$
x \succ^{\prime} y \Leftarrow x B z \succ^{\prime} y B z .
$$

Moreover, for any non-universal ${ }^{45} B \in \Sigma_{A}, x, y, z \in X$ such that $x, y \succeq z$,

$$
x \succ^{\prime} y \Leftarrow z B x \succ^{\prime} z B y .
$$

Proof. Consider an event $B \in \Sigma_{A}$, and $x, y, z \in X$ such that $x \succ^{\prime} y$. Notice that by construction this implies $\delta_{x} \succ \delta_{y}$. Notice also that the nonnull events in $\Sigma_{A}$ are $A, A^{C}$, and $\Omega^{\prime}$. In the case of $B=\Omega^{\prime}$ we have $x B z=x$ and $y B z=y$, which guarantees that $x \succ^{\prime} y$. Now consider the case in which $B=A$. By Claim $1, \gamma^{-1}(x A z)=\frac{1}{2} x+\frac{1}{2} z$ and $\gamma^{-1}(y A z)=\frac{1}{2} y+\frac{1}{2} z$. Since $\delta_{x} \succ \delta_{y}$, then $\frac{1}{2} x+\frac{1}{2} z \triangleright_{\text {FOSD }} \frac{1}{2} y+\frac{1}{2} z$, which, by Axiom 1 (FOSD), implies $\frac{1}{2} x+\frac{1}{2} z \succ \frac{1}{2} y+\frac{1}{2} z$; hence, $\gamma^{-1}(x A z) \succ \gamma^{-1}(y A z)$. By construction of $\succeq^{\prime}$ this implies $x A z \succ^{\prime} y A z$. Now consider the case in which $B=A^{c}$. This implies that we have $x A^{C} z=z A x$ and $y A^{C} z=z A y$. Notice, however, that by construction we must have $z A x \in \gamma\left(\frac{1}{2} x+\frac{1}{2} z\right)$. Since we also have $x A z \in \gamma\left(\frac{1}{2} x+\frac{1}{2} z\right)$, by construction of $\succeq^{\prime}$ we must have $z A x \sim^{\prime} x A z$. Similarly, we have $z A y \sim^{\prime} y A z$. We have already proved that we must have $x A z \succ y A z$, and this, by transitivity, implies $z A A \succ^{\prime} z A y$ as sought.

\footnotetext{
${ }^{44}$ We recall that an event $E$ is essential if we have $x \succ^{\prime} x A y \succ^{\prime} y$ for some $x, y \in X$.

${ }^{45}$ An event is universal if $y \sim x A y$ for all $x, y \in X$ such that $x \succ y$.
} 
Now consider some $B \in \Sigma_{A}$ that is non-universal. If $B=\varnothing$, we trivially have that $x \succ^{\prime}$ $y \Leftarrow z B x \succ^{\prime} z B y$. Now consider the case in which $B=A$. In this case we have $x \succ^{\prime} y$ and we need to show $z A x \succ^{\prime} z A y$ : but this is exactly what we have shown above. Similarly, when $B=A^{C}$, we need to show that if $x \succ^{\prime} y$, then $x A z \succ^{\prime} y A x$, which is again exactly what we have shown before.

Claim 3 ( $A$ Continuity). Let $\left\{g_{\alpha}^{\prime}\right\}_{\alpha \in D} \subseteq \mathcal{F}_{A}^{\prime}$ be a net that pointwise converges to $g^{\prime}$. For every $f^{\prime} \in \mathcal{F}^{\prime}$, if $g_{\alpha}^{\prime} \succeq^{\prime} f$ (resp. $f \succeq^{\prime} g_{\alpha}^{\prime}$ ) for all $\alpha \in D$, then $g^{\prime} \succeq^{\prime} f^{\prime}$ (resp. $f^{\prime} \succeq^{\prime} g^{\prime}$ ).

Proof. This claim is a trivial consequence of the continuity of $\succeq$ and of the continuity results of $\succeq^{\prime}$ proved in Step 2 above. To see why, consider $f^{\prime}, g^{\prime} \in \mathcal{F}^{\prime}$ and a net $\left\{g_{\alpha}^{\prime}\right\}_{\alpha \in D} \subseteq$ $\mathcal{F}_{A}^{\prime}$ that pointwise converges to $g^{\prime}$ such that $g_{\alpha}^{\prime} \succeq^{\prime} f^{\prime}$ for all $\alpha \in D$. By construction we must have $\gamma^{-1}\left(g_{\alpha}^{\prime}\right) \succeq \gamma^{-1}\left(f^{\prime}\right)$. Now, notice that if $g_{\alpha}^{\prime}$ pointwise converges to some $g^{\prime}$, then we must have that $\gamma^{-1}\left(g_{\alpha}^{\prime}\right)$ converges to $\gamma^{-1}\left(g^{\prime}\right)$ by Step 2 above. But then, by continuity of $\succeq$ (Axiom 3), we must have $\gamma^{-1}\left(g^{\prime}\right) \succeq \gamma^{-1}\left(f^{\prime}\right)$ and, therefore, $g^{\prime} \succeq^{\prime} f^{\prime}$ as sought. The proof of the opposite case ( $f \succeq^{\prime} g_{\alpha}^{\prime}$ for all $\alpha \in D$ ) is analogous.

Claim 4 ( $A$ Substitution). For any $x, y, z^{\prime}, z^{\prime \prime} \in X$ and $B, C \in \Sigma_{A}$ such that $x \succeq^{\prime} z^{\prime} \succeq^{\prime} y$ and $x \succeq^{\prime} z^{\prime \prime} \succeq^{\prime} y$, we have

$$
c e_{x B z^{\prime}}^{\prime} C c e_{z^{\prime \prime} B y}^{\prime} \sim^{\prime} c e_{x C z^{\prime \prime}}^{\prime} B c e_{z^{\prime} C y}^{\prime} .
$$

Proof. Consider first the case in which $B=\varnothing$. In this case, the claim becomes $c e_{z^{\prime}}^{\prime} C c e_{y}^{\prime} \sim^{\prime} c e_{z^{\prime} C y}^{\prime}$, which is trivially true. The case $C=\varnothing$ is analogous. Now consider the case $B=\Omega^{\prime}$. The claim becomes $c e_{x}^{\prime} C c e_{z^{\prime \prime}}^{\prime} \sim^{\prime} c e_{x C z^{\prime \prime}}^{\prime}$, which again is trivially true. The case in which $C=\Omega^{\prime}$ is again analogous.

We are left with the case in which $B=A$ and $C=A^{C}$. (The case $B=A^{C}$ and $C=A$ is again analogous.) In this case the claim becomes $c e_{x A z^{\prime}}^{\prime} A^{C} c e_{z^{\prime \prime} A y}^{\prime} \sim^{\prime} c e_{x A^{C} z^{\prime \prime}}^{\prime} A c e_{z^{\prime} C y^{\prime}}^{\prime}$ which is equivalent to $c e_{z^{\prime \prime} A y}^{\prime} A c e_{x A z^{\prime}}^{\prime} \sim^{\prime} c e_{z^{\prime \prime} A x}^{\prime} A c e_{y A z^{\prime}}^{\prime}$. Now notice that since $c e_{x A y}^{\prime} \in X$ for all $x, y \in X$, by Claim 1 , we have that $\gamma^{-1}\left(c e_{z^{\prime \prime} A y}^{\prime} A c e_{x A z^{\prime}}^{\prime}\right)=\frac{1}{2} c e_{z^{\prime \prime} A y}^{\prime}+\frac{1}{2} c e_{x A z^{\prime}}^{\prime}$. At the same time, consider some $r, s \in X$ and notice that, since $c e_{r A s}^{\prime} \sim^{\prime} r A s$ by construction, then we must have $\gamma^{-1}\left(c e_{r A s}^{\prime}\right) \sim \gamma^{-1}(r A s)$. Since $\gamma^{-1}(r A s)=\frac{1}{2} r+\frac{1}{2} s$ again by Claim 1, then we have that $\gamma^{-1}\left(c e_{r A s}^{\prime}\right) \sim \frac{1}{2} r+\frac{1}{2} s$. Moreover, since $c e_{r A s}^{\prime} \in X$, then we must have that $\delta_{c e^{\prime} A s} \sim \delta_{c_{\frac{1}{2} z+\frac{1}{2} s}}$. Since this is true for all $r, s \in X$, then by Axiom 1 (FOSD) we must have $\frac{1}{2} c_{\frac{1}{2} z^{\prime \prime}+\frac{1}{2} y}+\frac{1}{2} c_{\frac{1}{2} x+\frac{1}{2} z^{\prime}} \sim \frac{1}{2} c e_{z^{\prime \prime} A y}^{\prime}+\frac{1}{2} c e_{x A z^{\prime}}^{\prime}$; hence, $\gamma^{-1}\left(c e_{z^{\prime \prime} A y}^{\prime} A c e_{x A z^{\prime}}^{\prime}\right) \sim \frac{1}{2} c_{\frac{1}{2} z^{\prime \prime}+\frac{1}{2} y}+$ $\frac{1}{2} c_{\frac{1}{2} x+\frac{1}{2} z^{\prime}}$. By analogous arguments, we must have $\gamma^{-1}\left(c e_{z^{\prime \prime} A x}^{\prime} A c e_{y A z^{\prime}}^{\prime}\right) \sim \frac{1}{2} c_{\frac{1}{2} z^{\prime \prime}+\frac{1}{2} x}+$ $\frac{1}{2} c_{\frac{1}{2} y+\frac{1}{2} z^{\prime}}$. At the same time, by Axiom 4 we must have $c_{\frac{1}{2} z^{\prime \prime}+\frac{1}{2} y}+\frac{1}{2} c_{\frac{1}{2} x+\frac{1}{2} z^{\prime}} \sim \frac{1}{2} c_{\frac{1}{2}} z^{\prime \prime}+\frac{1}{2} x+$ $\frac{1}{2} c_{\frac{1}{2} y+\frac{1}{2} z^{\prime}}$, which by transitivity implies $\gamma^{-1}\left(c e_{z^{\prime \prime} A y}^{\prime} A c e_{x A z^{\prime}}^{\prime}\right) \sim \gamma^{-1}\left(c e_{z^{\prime \prime}}^{\prime \prime} A x A c e_{y A z^{\prime}}^{\prime}\right)$; hence, $c e_{z^{\prime \prime} A y}^{\prime} A c e_{x A z^{\prime}}^{\prime} \sim c e_{z^{\prime \prime}}^{\prime} A x A c e_{y A z^{\prime}}^{\prime}$ as sought.

This proves that $\succeq^{\prime}$ is locally bi-separable in the sense of Ghirardato and Marinacci (2001). 
Step 4. We now prove that $\succeq^{\prime}$ admits a representation similar to MMEU. First of all, note that for any $f^{\prime}, g^{\prime} \in \mathcal{F}^{\prime}, x \in X$, and $\alpha \in(0,1)$,

$$
f^{\prime} \sim^{\prime} g^{\prime} \Rightarrow \alpha f^{\prime} \oplus(1-\alpha) x \sim^{\prime} \alpha g^{\prime} \oplus(1-\alpha) x .
$$

To see why, consider $f^{\prime}, g^{\prime} \in \mathcal{F}^{\prime}$ such that $f^{\prime} \sim^{\prime} g^{\prime}$. Notice that we could have $f^{\prime} \sim^{\prime} g^{\prime}$ in two possible cases: (i) $\gamma^{-1}\left(f^{\prime}\right)=\gamma^{-1}\left(g^{\prime}\right)$; (ii) $\gamma^{-1}\left(f^{\prime}\right) \neq \gamma^{-1}\left(g^{\prime}\right)$ but $\gamma^{-1}\left(f^{\prime}\right) \sim \gamma^{-1}\left(g^{\prime}\right)$. In either case, we must have $\gamma^{-1}\left(f^{\prime}\right) \sim \gamma^{-1}\left(g^{\prime}\right)$. By Axiom 6, then, we must have that for any $x \in X$ and $\alpha \in(0,1), \alpha \gamma^{-1}\left(f^{\prime}\right) \oplus(1-\alpha) \delta_{x} \sim \alpha \gamma^{-1}\left(g^{\prime}\right) \oplus(1-\alpha) \delta_{x}$. Let us now consider $\alpha \gamma^{-1}\left(f^{\prime}\right) \oplus(1-\alpha) \delta_{x}$ and notice that, by construction, we must have that $f^{\prime} \oplus$ $(1-\alpha) x \in \gamma\left(\alpha \gamma^{-1}\left(f^{\prime}\right) \oplus(1-\alpha) \delta_{x}\right)$ : in fact, we must have that for every $\omega \in \Omega$ and every $y \in X,\left(\alpha \gamma^{-1}\left(f^{\prime}\right) \oplus(1-\alpha) \delta_{x}\right)(\omega)(\alpha y \oplus(1-\alpha) x)=\ell\left(f^{\prime}(\omega)^{-1}(\alpha y \oplus(1-\alpha) x)\right)$. In turn, this means that $\gamma^{-1}\left(f^{\prime} \oplus(1-\alpha) x\right)=\alpha \gamma^{-1}\left(f^{\prime}\right) \oplus(1-\alpha) \delta_{x}$. Similarly, $g^{\prime} \oplus(1-\alpha) x \in$ $\gamma\left(\alpha \gamma^{-1}\left(g^{\prime}\right) \oplus(1-\alpha) \delta_{x}\right)$ and $\gamma^{-1}\left(g^{\prime} \oplus(1-\alpha) x\right)=\alpha \gamma^{-1}\left(g^{\prime}\right) \oplus(1-\alpha) \delta_{x}$. Since we have $\alpha \gamma^{-1}\left(f^{\prime}\right) \oplus(1-\alpha) \delta_{x} \sim \alpha \gamma^{-1}\left(g^{\prime}\right) \oplus(1-\alpha) \delta_{x}$, then by transitivity $\gamma^{-1}\left(f^{\prime} \oplus(1-\alpha) x\right) \sim$ $\gamma^{-1}\left(g^{\prime} \oplus(1-\alpha) x\right)$; hence, $f^{\prime} \oplus(1-\alpha) x \sim^{\prime} g^{\prime} \oplus(1-\alpha) x$ as sought.

Claim 5. For any $f^{\prime}, g^{\prime} \in \mathcal{F}^{\prime}$ such that $f^{\prime} \sim^{\prime} g^{\prime}$, we have $\frac{1}{2} f^{\prime} \oplus \frac{1}{2} g^{\prime} \succeq^{\prime} f^{\prime}$.

Proof. Consider $f^{\prime}, g^{\prime} \in \mathcal{F}^{\prime}$ such that $f^{\prime} \sim^{\prime} g^{\prime}$. This can hold in two possible cases: (i) $\gamma^{-1}\left(f^{\prime}\right)=\gamma^{-1}\left(g^{\prime}\right)$; (ii) $\gamma^{-1}\left(f^{\prime}\right) \neq \gamma^{-1}\left(g^{\prime}\right)$ but $\gamma^{-1}\left(f^{\prime}\right) \sim \gamma^{-1}\left(g^{\prime}\right)$. In either case, we must have $\gamma^{-1}\left(f^{\prime}\right) \sim \gamma^{-1}\left(g^{\prime}\right)$. Consider the act $\frac{1}{2} f^{\prime} \oplus \frac{1}{2} g^{\prime}$ and notice that, by construction, for all $\omega \in \Omega$ we have $\left(\frac{1}{2} f^{\prime}(\omega, \cdot) \oplus \frac{1}{2} g^{\prime}(\omega, \cdot)\right)(A)=\frac{1}{2} f^{\prime}(\omega, A) \oplus \frac{1}{2} g^{\prime}(\omega, A)$ for all $A \in \Sigma^{*}$. This means that $\gamma^{-1}\left(\frac{1}{2} f^{\prime}(\omega, \cdot) \oplus \frac{1}{2} g^{\prime}(\omega, \cdot)\right)$ is a constant act (a lottery in $\left.\Delta(X)\right)$ and that $\gamma^{-1}\left(\frac{1}{2} f^{\prime}(\omega, \cdot) \oplus \frac{1}{2} g^{\prime}(\omega, \cdot)\right) \in \gamma^{-1}\left(f^{\prime}(\omega, \cdot)\right) \oplus \gamma^{-1}\left(g^{\prime}(\omega, \cdot)\right)$ for all $\omega \in \Omega$. By construction of $\oplus$ in $\mathcal{F}^{\prime}$, it follows that $\gamma^{-1}\left(\frac{1}{2} f^{\prime} \oplus \frac{1}{2} g^{\prime}\right) \in \gamma^{-1}\left(f^{\prime-1}\left(g^{\prime}\right)\right)$. We have already established $\gamma^{-1}\left(f^{\prime}\right) \sim \gamma^{-1}\left(g^{\prime}\right)$. Thus by Axiom 5 (hedging) we must have $\gamma^{-1}\left(\frac{1}{2} f^{\prime} \oplus \frac{1}{2} g^{\prime}\right) \succeq \gamma^{-1}\left(f^{\prime}\right)$; hence, $\frac{1}{2} f^{\prime} \oplus \frac{1}{2} g^{\prime} \succeq^{\prime} f^{\prime}$ as sought.

Claim 6. There exists a continuous nonconstant function $u: X \rightarrow \mathbb{R}$ and a nonempty, weak*-compact, and convex set $P$ of finitely additive probabilities of $\Sigma^{\prime}$ such that $\succeq^{\prime}$ is represented by the functional

$$
V^{\prime}\left(f^{\prime}\right):=\min _{p \in P} \int_{\Omega^{\prime}} u\left(f^{\prime}\right) \mathrm{d} p .
$$

Moreover, $u$ is unique up to a positive affine transformation and $P$ is unique. Moreover, $|P|=1$ if and only if $\succeq^{\prime}$ is such that for any $f^{\prime}, g^{\prime} \in \mathcal{F}^{\prime}$ such that $f^{\prime} \sim^{\prime} g^{\prime}$ we have $\frac{1}{2} f^{\prime} \oplus$ $\frac{1}{2} g^{\prime} \sim^{\prime} f^{\prime}$.

Proof. This claim follows directly from Proposition 3 in Ghirardato et al. (2003), where the essential event for which axioms are defined is the event $A$ defined above. (It should be noted that weak* compactness of $P$ follows as well.) The last part of the theorem, which characterizes the case in which $|P|=1$, is a well known property of MMEU representations. (See Gilboa and Schmeidler 1989.) 
Step 5 . We now use the result above to provide a representation of the restriction of $\succeq$ to constant acts. To this end, let us first look at the restriction of $\succeq^{\prime}$ to acts in $\mathcal{F}^{\prime}$ that are constant in their first component: define $\mathcal{F}^{*} \subset \mathcal{F}^{\prime}$ as $\mathcal{F}^{*}:=\left\{f^{\prime} \in \mathcal{F}^{\prime}: f^{\prime}(\omega, \cdot)=\right.$ $f^{\prime}\left(\omega^{\prime}, \cdot\right)$ for all $\left.\omega, \omega^{\prime} \in \Omega\right\}$. Define by $\succeq^{*}$ the restriction of $\succeq^{\prime}$ to $\mathcal{F}^{*}$.

Claim 7. There exists a unique nonempty, closed, and convex set $\Phi$ of finitely additive probabilities over $\Sigma^{*}$ such that, for any enumeration of the support of $\left\{x_{1}, \ldots, x_{|\operatorname{supp}(p)|}\right\}$, the restriction of $\succeq$ to $\Delta(X)$ is represented by the functional

$$
V^{*}(p):=\min _{\phi \in \Phi} \sum_{i=1}^{|\operatorname{supp}(p)|} \phi\left(\left[\sum_{j=1}^{i-1} p\left(x_{j}\right), \sum_{j=1}^{i} p\left(x_{j}\right)\right]\right) u\left(x_{i}\right) .
$$

Proof. Following Claim 6 and defining $\Phi$ as the projection of $P$ on $[0,1]$, we obtain a nonempty, closed, and convex set $\Phi$ of finitely additive probabilities over $\Sigma^{*}$ such that $\succeq^{*}$ is represented by

$$
\hat{V}^{*}\left(f^{*}\right):=\min _{p \in \Phi} \int_{[0,1]} u\left(f^{*}(s)\right) \mathrm{d} p .
$$

Notice that by construction of $\gamma$ and by definition of $\mathcal{F}^{*}$, we must have that $\gamma(p) \subseteq \mathcal{F}^{*}$ for all $p \in \Delta(X)$. We will now argue that, for all $p, q \in \Delta(X)$, we have $p \succeq q$ if and only if $f^{*} \succeq^{*} g^{*}$ for some $f^{*}, g^{*} \in \mathcal{F}^{*}$ such that $\gamma^{-1}\left(f^{*}\right)=p$ and $\gamma^{-1}\left(g^{*}\right)=q$. To see why, notice that if $p \succeq q$, then we must have $f^{*} \succeq^{\prime} g^{*}$; hence, $f^{*} \geq^{*} g^{*}$. Conversely, suppose that we have $f^{*} \succeq^{*} g^{*}$ for some $f^{*}, g^{*} \in \mathcal{F}^{*}$ such that $\gamma^{-1}\left(f^{*}\right)=p$ and $\gamma^{-1}\left(g^{*}\right)=q$, but $q \succ p$. But then, by definition of $\succeq^{\prime}$, we should have $g^{*} \succ^{\prime} f^{*}$, a contradiction.

Notice now that for every $p \in \Delta(X)$, if $f^{*}, g^{*} \in \gamma(p)$, then we must have $\hat{V}\left(f^{*}\right)=$ $\hat{V}\left(g^{*}\right)$ : the reason is, by construction of $\succeq^{\prime}$, we must have $f^{*} \sim^{*}$; hence, $f^{*} \sim^{*} g^{*}$ and, hence, $\hat{V}\left(f^{*}\right)=\hat{V}\left(g^{*}\right)$. Define now $V: \Delta(X) \rightarrow \mathbb{R}$ as $V^{*}(p):=\hat{V}^{*}\left(f^{*}\right)$ for some $f^{*} \in \gamma(p)$. By the previous observation this is well defined. Now notice that we have $p \succeq q$ if and only if $f^{*} \succeq^{*} g^{*}$ for some $f^{*}, g^{*} \in \mathcal{F}^{*}$ such that $\gamma^{-1}\left(f^{*}\right)=p$ and $\gamma^{-1}\left(g^{*}\right)=q$, which holds if and only if $\hat{V}^{*}\left(f^{*}\right) \geq \hat{V}^{*}\left(g^{*}\right)$, which in turns hold if and only if $V^{*}(p) \geq V^{*}(q)$, which means that $V^{*}$ represents the restriction of $\succeq$ on $\Delta(X)$ as sought.

Notice now that $\succeq^{*}$ satisfies Arrow's monotone continuity axiom. That is, for any $f, g \in \mathcal{F}^{*}$ such that $f \succ^{*} g$, and for any $x \in X$ and sequence of events $E_{1}, \ldots, E_{n}$ in $\Sigma^{*}$ with $E_{1} \subseteq E_{2} \subseteq \cdots$ and $\bigcap_{n \geq 1} E_{n}=\varnothing$, there exists $\bar{n} \geq 1$ such that

$$
x E_{\bar{n}} f \succ^{*} g \text { and } f \succ^{*} x E_{\bar{n}} g .
$$

To prove it, consider $f, g, x$, and $E_{1}, \ldots$ as in the claim above. Notice first of all that for any $s \in \Omega^{\prime}$, there must exist some $\hat{n}$ such that for all $n \geq \hat{n}$ we have $s \notin E_{n}$ : otherwise, if this was not true for some $s \in \Omega^{\prime}$, we would have $s \in \bigcap_{n \geq 1} E_{n}$, a contradiction. In turn, this means that we have $x E_{n} f \rightarrow f$ pointwise: for any $s \in \Omega^{\prime}$, there must exist some $n$ such that $s \notin E_{n}$ and, therefore, $x E_{n} f(s)=f(s)$ as sought. Notice then that by the continuity properties of $\succeq^{\prime}$ proved in Step 2, we must therefore have that $\gamma^{-1}\left(x E_{n} f\right) \rightarrow \gamma^{-1}(f)$. We now show that we must have some $\bar{n}_{1} \geq 1$ such that $x E_{\bar{n}_{1}} f \succ^{*} g$ for all $n \geq \bar{n}_{1}$. Assume, 
by means of contradiction, that this is not the case: for every $n \geq 1$, there exists some $n^{\prime} \geq n$ such that $g \geq^{*} x E_{n}^{\prime} f$. Construct now the subsequence of $E_{1}, \ldots$ which includes these events, i.e., the events such that $g \succeq^{*} x E_{n^{\prime}}^{\prime} f$ : by the previous argument it must be a subsequence of $E_{1}, \ldots$ and we must have that $E_{1}^{\prime} \subseteq E_{2}^{\prime} \subseteq \cdots$ and $\bigcap_{n \geq 1} E_{n}^{\prime}=\varnothing$. This means that we have $g \succeq^{*} x E_{n}^{\prime} f$ for all $n$. By construction this then means that we have $\gamma^{-1}(g) \succeq \gamma^{-1}\left(x E_{n}^{\prime} f\right)$. Now consider $\gamma^{-1}\left(x E_{n}^{\prime} f\right)$ and notice that we have proved above that $\gamma^{-1}\left(x E_{n}^{\prime} f\right) \rightarrow \gamma^{-1}(f)$ as $n \rightarrow \infty$. By Axiom 3 (continuity), then we must have that $\gamma^{-1}(g) \succeq \gamma^{-1}(f)$, which in turns means that $g \succeq^{*} f$, a contradiction. An identical argument shows that there must exist $\bar{n}_{2} \geq 1$ such that $f \succ^{*} x E_{\bar{n}_{2}} g$ for all $n \geq \bar{n}_{2}$. Any $n \geq \max \left\{n_{1}, n_{2}\right\}$ will therefore give us the desired rankings.

Given that $\succeq *$ satisfies Arrow's monotone continuity axioms, Theorem 1 in Chateauneuf et al. (2005) implies that all measures in $\Phi$ are countably additive.

\section{Claim 8. The measures in $\Phi$ are atomless.}

Proof. We will first of all follow a standard approach and define the likelihood ranking induced by the $\succeq^{*}$. In particular, define $\succeq_{L}$ on $\Sigma^{*}$ as

$$
A \succeq_{L} B \Leftrightarrow \min _{\phi \in \Phi} \phi(A) \geq \min _{\phi \in \Phi} \phi(B) .
$$

Theorem 2 in Chateauneuf et al. (2005) shows that every $\phi \in \Phi$ is atomless if and only if for all $A \in \Sigma^{*}$ such that $A \succ_{L} \varnothing$, there exists $B \subseteq A$ such that $A \succ_{L} B \succ_{L} \varnothing, A \in \Sigma^{*}$ such that $A \succ_{L} \varnothing$, and notice that this implies that we have $\min _{\phi \in \Phi} \phi(A)>0$; hence, $\phi(A)>0$ for all $\phi \in \Phi$. Since every $\phi \in \Phi$ is mutually absolutely continuous with respect to the Lebesgue measure, this implies $\ell(A)>0$. Since $\ell$ is atomless, then there exists $B \subseteq A$ such that $\ell(A)>\ell(B)>0$. Notice that this implies $\ell(A \backslash B)>0$. Again since all $\phi \in$ $\Phi$ are mutually absolutely continuous with respect to the Lebesgue measure, we must therefore have $\phi(A)>0, \phi(A \backslash B)>0$ and $\phi(B)>0$ for all $\phi \in \Phi$. But this means that we have $\phi(A)=\phi(B)+\phi(A \backslash B)>\phi(B)>0$ for all $\phi \in \Phi$. But this implies $\min _{\phi \in \Phi} \phi(A)>$ $\min _{\phi \in \Phi} \phi(B)>0$; hence, $A \succ_{L} B \succ_{L} \varnothing$ as sought.

Claim 9. The Lebesgue measure $\ell$ belongs to $\Phi$. Moreover, all measures in $\Phi$ are mutually absolutely continuous and, in particular, they are all mutually absolutely continuous with respect to the Lebesgue measure $\ell$.

Proof. We will first prove that the Lebesgue measure $\ell$ belongs to $\Phi$. Assume by means of contradiction that $\ell \notin \Phi$. By the uniqueness of $\Phi$, we know that there must therefore exist some $f \in \mathcal{F}^{*}$ such that $\hat{V}^{*}(f):=\min _{p \in \Phi} \int_{[0,1]} u(f(s)) \mathrm{d} p>\int_{[0,1]} u(f(s)) \mathrm{d} \ell$. Call $p_{1}$ a generic element of $\arg \min _{p \in \Phi} \int_{[0,1]} u(f(s)) \mathrm{d} p$. Notice that since $\int_{[0,1]} u\left(f^{*}(s)\right) \mathrm{d} p_{1}>$ $\int_{[0,1]} u(f(s)) \mathrm{d} \ell$, it must be the case that $p_{1}(A)>\ell(A)$ for some $A \subset[0,1]$ such that $u(f(A))>\int_{[0,1]} u\left(f^{*}(s)\right) \mathrm{d} \ell$, and that $p_{1}(B)<\ell(B)$ for some $B \subset[0,1]$ such that $u(f(B))<u(f(A))$.

Suppose first of all that $\ell(A) \geq \ell(B)$. Now consider some $f^{\prime} \in \mathcal{F}^{*}$ constructed as follows. Consider any $C \subseteq A$ such that $\ell(C)=\ell(B)$ and $p_{1}(C)>\ell(C)$. (This must be 
possible since $p_{1}(A)>\ell(A)$.) Notice that we must therefore have $p_{1}(C)>p_{1}(B)$ since $p_{1}(C)>\ell(C)=\ell(B)>p_{1}(B)$. Now construct the act $f^{\prime}$ as $f^{\prime}(s)=f(s)$ if $s \notin C \cup B$; $f^{\prime}(C)=f(B)$ and $f^{\prime}(B)=f(A)$. (Notice that what we have done is that we have moved the "bad" outcomes to some events to which $p_{1}$ assigns a likelihood above the Lebesgue measure, while we have moved the "good" outcomes to some event to which $p_{1}$ assigns a likelihood below the Lebesgue measure.) Notice now that, by construction, we must have that $f, f^{\prime} \in \gamma(p)$ for some $p \in \Delta(X)$; hence, we must have $f \sim^{*} f^{\prime}$. At the same time, since $p_{1}(C)>p_{1}(B)$ and since $u(f(B))<u(f(A))=u(f(C))$, we must also have $\hat{V}^{*}(f)=\int_{[0,1]} u(f(s)) \mathrm{d} p_{1}>\int_{[0,1]} u\left(f^{\prime}(s)\right) \mathrm{d} p \geq \min _{p \in \Phi} \int_{[0,1]} u\left(f^{\prime}(s)\right) \mathrm{d} p=\hat{V}^{*}\left(f^{\prime}\right)$. But this means that we have $\hat{V}^{*}(f)>\hat{V}^{*}\left(f^{\prime}\right)$; hence, $f \succ f^{\prime}$, contradicting $f \sim^{*} f^{\prime}$. The proof for the case in which $\ell(A)<\ell(B)$ is specular.

We now turn to prove that all measures in $\Phi$ are mutually absolutely continuous and, in particular, they are all mutually absolutely continuous with respect to the Lebesgue measure $\ell$. To prove this, we will prove that for every event $E$ in $[0,1], E$ is null for $\succeq^{*}$ if and only if $\ell(E)=0 .{ }^{46}$ In turn this means that all measures are mutually absolutely continuous with respect to each other.

Consider some measurable $E \subset[0,1]$ such that $\ell(E)=0$. Suppose, by means of contradiction, that $\{\phi \in \Phi: \phi(E)>0\} \neq \varnothing$. Then consider any $x, y \in X$ such that $\delta_{x} \succ \delta_{y}$ (which must exist by nontriviality) and construct the act $y E x \in \mathcal{F}^{*}$. Since $\{\phi \in$ $\Phi: \phi(E)>0\} \neq \varnothing$, then we must have that $\min _{\phi \in \Phi} \phi(E) u(y)+(1-\phi(E)) u(x)<u(x)$, which in turns means that $y E x \prec^{*} x$ (by Claim 7); hence, $y E x \prec^{\prime} x$. However, notice that, since $\ell(E)=0$, we must have that $\gamma^{-1}(y E x)=\delta_{x}=\gamma^{-1}(x)$. By construction of $\succeq^{\prime}$, then we must have $y E x \sim^{\prime} x$, contradicting $y E x \prec^{\prime} x$.

Consider now some measurable $E \subset[0,1]$ such that $\ell(E)>0$. We now want to show that $\phi(E)>0$ for all $\phi \in \Phi$. Suppose, by means of contradiction, that $\{\phi \in$ $\Phi: \phi(E)=0\} \neq \varnothing$. Then consider any $x, y \in X$ such that $\delta_{x} \succ \delta_{y}$ (which must exist by nontriviality) and construct the act $x E y \in \mathcal{F}^{*}$. Since $\{\phi \in \Phi: \phi(E)=0\} \neq \varnothing$, then we must have that $\min _{\phi \in \Phi} \phi(E) u(y)+(1-\phi(E)) u(x)=u(y)$, which in turns means that $x E y \sim^{*} y$ (by Claim 7); hence, $x E y \sim^{\prime} y$. However, notice that since $\ell(E)>0$, then $\gamma^{-1}(x E y) \triangleright_{\text {FOSD }} \gamma^{-1}(y)$, which implies that we must have $\gamma^{-1}(x E y) \succ \gamma^{-1}(y)$ by Axiom 2 (monotonicity), which implies $x E y \succ^{\prime} y$ by construction of $\succeq^{\prime}$, contradicting $x E y \sim^{\prime} y . \triangleleft$

Finally, notice that $\Phi$ is weak compact. To see why, note that we already know that $\Phi$ is weak* compact. At the same time, we also know that every element in $\Phi$ is countably additive: we can then apply Lemma 3 in Chateauneuf et al. (2005) to prove the desired result. (Notice that this argument could be also derived from standard Banach lattice techniques: as cited by Chateauneuf et al. 2005 one could follow Aliprantis and Burkinshaw 2006, especially Section 4.2.)

Step 6. We now derive the main representation. First of all, define as $\hat{\mathcal{F}}$ the subset of acts in $\mathcal{F}^{\prime}$ that are constant in the second component: $\hat{\mathcal{F}}:=\left\{f^{\prime} \in \mathcal{F}^{\prime}: f^{\prime}(\omega,[0,1])=\right.$ $x$ for some $x \in X$ \}. Define $\hat{\succeq}$ to be the restriction of $\succeq$ to $\hat{\mathcal{F}}$. Now notice that there exists

\footnotetext{
${ }^{46}$ Recall that in this case we can define null events by saying that an event $E$ is null if and only if $\phi(E)=0$ for some $\phi \in \Phi$.
} 
a convex and compact set of finitely additive probability measures $\Pi$ on $\Omega$, such that $\hat{\succeq}$ is represented by the functional

$$
\hat{V}(\hat{f}):=\min _{\pi \in \Pi} \int_{\Omega} \pi(\omega) u(\hat{f}(\omega,[0,1])) \mathrm{d} \omega .
$$

Moreover, $\Pi$ is unique. Again, this trivially follows from Claim 6 , where $\Pi$ is the projection of $P$ on $\Omega$.

Notice that from Axiom 3 (continuity) and Axiom 1 (FOSD) it trivially follows that for any $p \in \Delta(X)$ there exists one $x \in X$ such that $\delta_{x} \sim p$. Thus $c e(p)$ is well defined for all $p \in \Delta(X)$. Now, for any act $f$, construct the act $\bar{f} \in \mathcal{F}$ as $\bar{f}(\omega):=\delta_{c_{f(\omega)}}$. Notice that for any $f, g \in \mathcal{F}$, we must have $f \succeq g$ if and only if $\bar{f} \succeq \bar{g}$ by Axiom 2 (monotonicity). At the same time, notice that, by construction of $\gamma$, for every $f \in \mathcal{F},|\gamma(\bar{f})|=1$ and $\gamma(\bar{f})(\omega,[0,1])=\delta_{c_{f(\omega)}}$. This means also that $\gamma(\bar{f}) \in \hat{\mathcal{F}}$ for all $f, g \in \mathcal{F}$. In turn, we must have that for all $f, g \in \mathcal{F}, f \succeq g$ if and only if $\gamma(\bar{f}) \succeq^{\prime} \gamma(\bar{g})$, which is equivalent to $\gamma(\bar{f}) \hat{\succeq} \gamma(\bar{g})$, which we know is true if and only if $\min _{\pi \in \Pi} \int_{\Omega} \pi(\omega) u(\gamma(\bar{f})(\omega,[0,1])) \mathrm{d} \omega \geq$ $\min _{\pi \in \Pi} \int_{\Omega} \pi(\omega) u(\gamma(\bar{f})(\omega,[0,1])) \mathrm{d} \omega$. At the same time, we know that for each $f \in \mathcal{F}$, we have that $\gamma(\bar{f})(\omega,[0,1])=\delta_{c_{f(\omega)}}$. In turn, this means that we have

$$
f \succeq g \quad \Leftrightarrow \quad \min _{\pi \in \Pi} \int_{\Omega} \pi(\omega) u\left(\delta_{c_{f(\omega)}}\right) \mathrm{d} \omega \geq \min _{\pi \in \Pi} \int_{\Omega} \pi(\omega) u\left(\delta_{c_{g(\omega)}}\right) \mathrm{d} \omega .
$$

At the same time, from Claim 7, we know that for all $p \in \Delta(X), u\left(c_{p}\right)=V^{*}\left(\delta_{c e_{p}}\right)=V^{*}(p)$, where the first equality holds by construction of $V^{*}$, while the second equality holds because $V^{*}$ represents the restriction of $\succeq$ to $\Delta(X)$ and because $c e_{p} \sim p$ for all $p \in \Delta(X)$. Given the definition of $V^{*}$ above, therefore, we obtain that $\succeq$ is represented by the functional

$$
V(f):=\min _{\pi \in \Pi} \int_{\Omega} \pi(\omega) \min _{\phi \in \Phi} \sum_{i=1}^{|\operatorname{supp}(p)|} \phi\left(\left[\sum_{j=1}^{i-1} p\left(x_{j}\right), \sum_{j=1}^{i} p\left(x_{j}\right)\right]\right) u\left(x_{i}\right) \mathrm{d} \omega,
$$

which is the desired representation. (The uniqueness properties have been proved in the various steps.) Finally, notice that if $\succeq$ satisfies Axiom 8, then we must have that $\succeq^{\prime}$ is such that for any $f^{\prime}, g^{\prime} \in \mathcal{F}^{\prime}$ such that $f^{\prime} \sim^{\prime} g^{\prime}$ we have $\frac{1}{2} f^{\prime} \oplus \frac{1}{2} g^{\prime} \sim^{\prime} f^{\prime}$. But then, by Claim 6 we have that $|P|=1$, which implies $|\Pi|=|\Phi|=1$. Moreover, since $\ell \in \Phi$, we must therefore have $\Phi=\{\ell\}$.

Step 7 . We now show that, without loss of generality we can restict our attention to decreasing, measure-preserving maps $\mu$. In doing so, we show that the resulting set of priors has the property of being state increasing.

Let us consider a measure-preserving function $\mu: \Delta(X) \rightarrow[0,1]^{X}$ that is decreasing (in the sense of Definition 9) and convex: i.e., for any $p \in \Delta(X)$ and for any $x \in X, \mu^{-1}(x)$ is convex.

We now define a binary relation $B$ on $\Phi$ as follows: for any $\phi, \phi^{\prime} \in \Phi$, we have $\phi B \phi^{\prime}$ if and only if $\int_{[0,1]} u(\mu(p)) \mathrm{d} \phi \leq \int_{[0,1]} u(\mu(p)) \mathrm{d} \phi^{\prime}$ for all $p \in \Delta(X)$. Notice that the relation $B$ depends on both $u$ and $\mu$; notice, moreover, that we have $\phi B \phi^{\prime}$ and $\phi^{\prime} B \phi$ if and only if $\phi=\phi^{\prime}$, which means that $B$ is reflexive. Finally, notice that $B$ is also transitive by construction. 
Claim 10. The relation $B$ is upper semicontinuous when $B$ is metrized using the weak metric. That is, for any $\left(\phi_{m}\right) \in \Phi^{\infty}$ and $\phi, \phi^{\prime} \in \Phi$, if $\phi_{m} \rightarrow \phi^{\prime}$ weakly and $\phi_{m} B \phi$ for all $m$, then $\phi^{\prime} B \phi$.

Proof. Suppose that we have $\phi_{m}, \phi$, and $\phi^{\prime}$ as in the statement of the claim. This means that for any $p \in \Delta(X)$ we have $\int_{[0,1]} u(\mu(p)) \mathrm{d} \phi_{m} \leq \int_{[0,1]} u(\mu(p)) \mathrm{d} \phi$. Notice moreover that, by construction of $\mu$, there must exist $x_{1}, \ldots, x_{n} \in X$ and $y_{0}, \ldots, y_{n} \in$ $[0,1]$, where $y_{0}=0$ and $y_{n}=1$, such that $\mu(p)(y)=x_{i}$ for all $y \in\left[y_{i-1}, y_{i}\right]$ for $i=1, \ldots, n$. In turn, this means that for any $\bar{\phi} \in \Phi$, we have $\int_{[0,1]} u(\mu(p)) \mathrm{d} \bar{\phi}=\sum_{i=1}^{n} u\left(x_{i}\right) \bar{\phi}\left(\left[y_{i-1}, y_{i}\right]\right)$. This means that we have $\sum_{i=1}^{n} u\left(x_{i}\right) \phi_{m}\left(\left[y_{i-1}, y_{i}\right]\right) \leq \sum_{i=1}^{n} u\left(x_{i}\right) \phi\left(\left[y_{i-1}, y_{i}\right]\right)$. At the same time, recall that $\phi^{\prime}$ is absolutely continuous with respect to the Lebesgue measure: this means that, by the Portmanteau theorem, ${ }^{47}$ since $\phi_{m} \rightarrow \phi^{\prime}$ weakly, then we must have that $\phi_{m}\left(\left[y_{i-1}, y_{i}\right]\right) \rightarrow \phi^{\prime}\left(\left[y_{i-1}, y_{i}\right]\right)$ for $i=1, \ldots, n$. But this means that we have $\sum_{i=1}^{n} u\left(x_{i}\right) \phi_{m}\left(\left[y_{i-1}, y_{i}\right]\right) \rightarrow \sum_{i=1}^{n} u\left(x_{i}\right) \phi^{\prime}\left(\left[y_{i-1}, y_{i}\right]\right)$; hence, $\sum_{i=1}^{n} u\left(x_{i}\right) \phi^{\prime}\left(\left[y_{i-1}, y_{i}\right]\right) \leq$ $\sum_{i=1}^{n} u\left(x_{i}\right) \phi\left(\left[y_{i-1}, y_{i}\right]\right)$, so $\int_{[0,1]} u(\mu(p)) \mathrm{d} \phi^{\prime} \leq \int_{[0,1]} u(\mu(p)) \mathrm{d} \phi$. Since this must be true for any $p \in \Delta(X)$, we therefore have $\phi^{\prime} B \phi$ as sought.

Now define the set $\operatorname{MAX}(\Phi, B):=\left\{\phi \in \Phi: \nexists \phi^{\prime} \in \Phi\right.$ s.t. $\phi^{\prime} B \phi$ and $\left.\phi^{\prime} \neq \phi\right\}$. Note that $\operatorname{MAX}(\Phi, B) \neq \varnothing$. This follows from noticing that since $\Phi$ is weak compact and $B$ is upper semi-continuous (in the weak metric), then standard results in order theory show that $\operatorname{MAX}(\Phi, B) \neq \varnothing$ : see, for example, Theorem 3.2.1 in Ok (2011).

Claim 11. For any $p \in \Delta(X)$ we have

$$
\min _{\phi \in \operatorname{MAX}(\Phi, B)} \int_{[0,1]} u(\mu(p)) \mathrm{d} \phi=\min _{\phi \in \Phi} \int_{[0,1]} u(\mu(p)) \mathrm{d} \phi .
$$

Proof. Since by construction $\operatorname{MAX}(\Phi, B) \subseteq \Phi$, it trivially follows that the right hand side of the equation is smaller than or equal to the left hand side for all $p \in \Delta(X)$. We are left to prove the converse. To this end, say by means of contradiction that there exist some $p \in \Delta(X)$ and some $\hat{\phi} \in \Phi \backslash \operatorname{MAX}(\Phi, B)$ such that $\int_{[0,1]} u(\mu(p)) \mathrm{d} \hat{\phi}<$ $\min _{\phi \in \operatorname{MAX}(\Phi, B)} \int_{[0,1]} u(\mu(p)) \mathrm{d} \phi$. This means that we cannot have $\phi^{\prime} B \hat{\phi}$ for any $\phi^{\prime} \in$ $\operatorname{MAX}(\Phi, B)$. Since $B$ is transitive, we must therefore have that $\hat{\phi} \in \operatorname{MAX}(\Phi, B)$, a contradiction.

Define the set $\Phi^{\prime}:=\left\{\phi \in \operatorname{MAX}(\Phi, B): \phi \in \arg \min _{\phi \in \operatorname{MAX}(\Phi, B)} \int_{[0,1]} u(\mu(p)) \mathrm{d} \phi\right.$ for some $p \in \Delta(X)\}$. We now define the notion of state-increasing priors.

Definition 11. A prior $\phi$ on [0,1] is state-increasing if there do not exist any $x_{1}, x_{2}, x_{3}$, $x_{4}$ such that $x_{1}<x_{2}<x_{3}<x_{4}, \ell\left(\left[x_{1}, x_{2}\right]\right)=\ell\left(\left[x_{3}, x_{4}\right]\right)$, and $\phi\left(\left[x_{1}, x_{2}\right]\right)>\phi\left(\left[x_{3}, x_{4}\right]\right)$.

Claim 12. Every prior $\phi \in \Phi^{\prime}$ is state-increasing.

\footnotetext{
${ }^{47}$ See Billingsley (1995, Chapter 5).
} 
Proof. Suppose by means of contradiction that there exists $\phi^{\prime} \in \Phi^{\prime}$ that is not statedecreasing. This means that there exist $x_{1}, x_{2}, x_{3}, x_{4}$ such that $x_{1}<x_{2}<x_{3}<x_{4}$, $\ell\left(\left[x_{1}, x_{2}\right]\right)=\ell\left(\left[x_{3}, x_{4}\right]\right)$ and $\phi^{\prime}\left(\left[x_{1}, x_{2}\right]\right)>\phi^{\prime}\left(\left[x_{3}, x_{4}\right]\right)$. Now notice the following. If we have a MP-MD representation, then for any measure-preserving map $\mu^{\prime}: \Delta(X) \rightarrow$ $[0,1]^{X}$ we must have

$$
\min _{\phi \in \Phi} \int_{[0,1]} u(\mu(p)) \mathrm{d} \phi=u\left(c e_{p}\right)=\min _{\phi \in \Phi} \int_{[0,1]} u\left(\mu^{\prime}(p)\right) \mathrm{d} \phi
$$

for all $p \in \Delta(X)$, for any $c e_{p} \in X$ such that $\delta_{c e_{p}} \sim p$. Since this must be true for every measure-preserving $\mu^{\prime}$ and for every $p$, then there must exist some $\hat{\phi} \in \Phi$ such that $\phi^{\prime}(A)=\hat{\phi}(A)$ for all $A \subset[0,1]$ such that $A \cap\left(\left[x_{1}, x_{2}\right] \cup\left[x_{3}, x_{4}\right]\right)=\varnothing$, and $\hat{\phi}\left(\left[x_{1}, x_{2}\right]\right)=$ $\phi^{\prime}\left(\left[x_{3}, x_{4}\right]\right)$ and $\hat{\phi}\left(\left[x_{3}, x_{4}\right]\right)=\phi^{\prime}\left(\left[x_{1}, x_{2}\right]\right)$ : the reason is that if we take a measurepreserving map $\mu^{\prime}$ that is identical to $\mu$ except that it maps to $\left[x_{3}, x_{4}\right]$ whatever $\mu$ maps to $\left[x_{1}, x_{2}\right]$ and vice versa, then there must exist a prior that minimizes the utility when $\mu^{\prime}$ is used and that returns exactly the same utility. Now notice that we must have that, by construction, $\phi^{\prime}\left(\left[x_{1}, x_{2}\right]\right)>\phi^{\prime}\left(\left[x_{3}, x_{4}\right]\right)$ and, hence, $\hat{\phi}\left(\left[x_{1}, x_{2}\right]\right)>\hat{\phi}\left(\left[x_{3}, x_{4}\right]\right)$, where $x_{1}<x_{2}<x_{3}<x_{4}, \ell\left(\left[x_{1}, x_{2}\right]\right)=\ell\left(\left[x_{3}, x_{4}\right]\right)$. (The two priors are otherwise the same.) But since $\mu$ assigns prizes with a higher utility to lower states, then this means that we have $\int_{[0,1]} u(\mu(p)) \mathrm{d} \hat{\phi} \leq \int_{[0,1]} u(\mu(p)) \mathrm{d} \phi^{\prime}$ for all $p \in \Delta(X)$. Since $\hat{\phi} \neq \phi^{\prime}$, therefore, we have that $\hat{\phi} B \phi^{\prime}$, which contradicts the fact that $\phi^{\prime} \in \Phi^{\prime} \subseteq \operatorname{MAX}(\Phi, B)$.

The above discussion implies that there exists a closed, weak compact subset $\Phi^{\prime \prime}$ of priors on $[0,1]$ such that every $\phi \in \Phi^{\prime \prime}$ is state-increasing, atomless, mutually absolutely continuous with respect to $\ell$ contains $\ell$, and such that $\succeq$ is represented by

$$
V(f):=\min _{\pi \in \Pi} \int_{\Omega} \pi(\omega) \bar{U}(f(\omega)) \mathrm{d} \omega,
$$

where $\bar{U}: \Delta(X) \rightarrow \mathbb{R}$ is defined as

$$
\bar{U}(p):=\min _{\phi \in \Phi^{\prime \prime}} \int_{[0,1]} \phi(s) u(\mu(p)) \mathrm{d} s,
$$

where $\mu$ is decreasing and $u$ and $\Pi$ are defined above. To see why, simply define the set $\Phi^{\prime \prime}$ as the closed convex hull of $\Phi^{\prime}$. Notice that this operation maintains the property that every $\phi$ in it is state-increasing and that it represents the preferences. Therefore, the result follows from Claims 11 and 12.

The set $\Phi^{\prime \prime}$ above might not be unique. ${ }^{48}$ However, we will now argue that there exists a unique minimal $\Phi^{\prime \prime}$, where by minimal we understand a representation with a set $\Phi^{\prime \prime}$ such that there is no $\hat{\Phi}^{\prime \prime} \subset \Phi^{\prime \prime}$ that represents the same preferences and satisfies all the properties required. Consider any minimal representation of the form above with a set of priors $\bar{\Phi}^{\prime \prime} \subseteq \Phi^{\prime \prime}$. To prove its uniqueness, assume by contradiction that there exists

\footnotetext{
${ }^{48}$ For example, if it does not already include it, one could add the identity function to the set or any convex combination of the identity function with any member of the set, and leave the representation unchanged.
} 
another minimal representation of the same preferences with a set of priors $\hat{\Phi}^{\prime \prime} \neq \bar{\Phi}^{\prime \prime}$. Now construct the set $H$ as the closed convex hull of $\left(\Phi \backslash \bar{\Phi}^{\prime \prime}\right) \cup \hat{\Phi}^{\prime \prime}{ }^{49}$ It is easy to see that we must have $H \neq \Phi$, since $\bar{\Phi}^{\prime \prime} \neq \hat{\Phi}^{\prime \prime}$ and by the fact that $\bar{\Phi}^{\prime \prime}$ is minimal. The key observation is then to notice that $(u, \Pi, H)$ is also a MP-MD representation of the same preferences (in the sense of (2), i.e., dropping the requirement of the mappings being decreasing). By the uniqueness of its properties we can assume that the utility function is the same. To see why, consider first a measure-preserving map $\mu^{\prime}$ that maps worse outcomes to higher states in $[0,1]$, as the map $\mu$ defined above. For any such map, for each lottery at least one of the minimizing priors must belong to $\bar{\Phi}^{\prime \prime}$ in the first representation, by construction. At the same time, the value of these acts computed using the worst prior in $\bar{\Phi}^{\prime \prime}$ must be equivalent to the value computed using the worst prior in $\hat{\Phi}^{\prime \prime}$, because both represent the same preferences in the representation above; hence, they must have the same certainty equivalents for each lottery. But then the minimizing priors in the second representation must belong to $\hat{\Phi}^{\prime \prime}$, and thus for any decreasing map, both are representations of the same preferences.

Let us now consider a map $\mu^{\prime \prime}$ that is not decreasing. Notice that for any such map in the representation of (2) we cannot have a lottery for which the minimizing prior belongs to $\bar{\Phi}^{\prime \prime}$. To see why, notice that if this was the case, we could also construct a lottery for which the minimizing prior also belongs to $\bar{\Phi}^{\prime \prime}$ (for the map at hand), but for which the value computed using a prior in $\bar{\Phi}^{\prime \prime}$ is strictly lower if we used a decreasing map (as $\mu^{\prime}$ above) instead of $\mu^{\prime \prime}$. The reason is that we can simply consider a lottery that is "fine enough," i.e., returns different outcomes with small probability, so that the fact that the $\mu^{\prime \prime}$ is not nonincreasing matters. (Recall that any prior in $\bar{\Phi}^{\prime \prime}$ assigns lower weight to lower states, which means that by using it we obtain lower values for maps that assign worse outcomes to higher states.) But this means that for this lottery we would obtain a strictly lower utility when we use a map like $\mu^{\prime}$ as opposed to when we use $\mu^{\prime \prime}$, which is impossible because the representation of (2) should be independent of the map used (the certainty equivalents must be the same). This proves that for any map $\mu^{\prime \prime}$, we cannot have that the unique minimizing prior belongs to $\bar{\Phi}^{\prime \prime}$. We now turn to argue that also for such a map two representations of the form of (2) must represent the same preferences. Given our last result, the only possibility for this not to be the case is that, for this map $\mu^{\prime \prime}$, there exists a lottery $p$ for which all the minimizing priors in the second representation belong to $\hat{\Phi}^{\prime \prime}$. We will now argue that this cannot be the case. If it were, then the value of $p$ computed using map $\mu^{\prime \prime}$ and a prior in $\hat{\Phi}^{\prime \prime}$ must be strictly below the value of $p$ computed using the same map and the worst prior in $\left(\Phi \backslash \bar{\Phi}^{\prime \prime}\right)$. And since we have proved that we cannot have that the unique minimizing prior for the first representation belongs to $\bar{\Phi}^{\prime \prime}$, then this means that the value of $p$ computed using map $\mu^{\prime \prime}$ and a prior in $\hat{\Phi}^{\prime \prime}$ is strictly below that computed using map $\mu^{\prime \prime}$ and the worst prior in $\Phi$. At the same time, notice that the value of $p$ computed using the worst prior in $\hat{\Phi}^{\prime \prime}$ and map $\mu^{\prime \prime}$ is weakly above that computed using the worst prior in $\hat{\Phi}^{\prime \prime}$ and a nonincreasing map like $\mu^{\prime}$ above. In turn, however, we have proved that for any such map, this must be equal to the value computed using the worst prior in $\bar{\Phi}^{\prime \prime}$; by construction, this

\footnotetext{
${ }^{49}$ Recall that $\Phi$ is the set of distortions of the MP-MD representation of the same preferences.
} 
must be weakly higher than the value assigned by the first representation when using map $\mu^{\prime}$. But this means that we have some $p$ such that the value assigned by the first representation when using map $\mu^{\prime}$ is strictly lower than the one assigned by the same representation when using map $\mu^{\prime \prime}$. This contradicts the fact that a representation of the form of (2) represents the same preferences regardless of the map, as these lotteries would have a different certainty equivalent depending on which map we use.

(3) $\Rightarrow$ (2). Consider a preference relation that admits a multiple priors and multiple distortions representation $(u, \Pi, \Phi)$. For every $\phi \in \Phi$ construct first the corresponding probability density function (PDF), $\mathrm{pdf}_{\phi}$. Notice that $\mathrm{pdf}_{\phi}$ is well defined since every $\phi$ is mutually absolutely continuous with respect to the Lebesgue measure (this follows from the Radon-Nikodym theorem, Aliprantis and Border 2006, Theorem 13.18). Moreover, notice that since every $\phi \in \Phi$ is state-increasing, then every $\operatorname{pdf}_{\phi}$ is a nondecreasing function in $[0,1]$. Moreover, since every $\phi \in \Phi$ is mutually absolutely continuous with respect to the Lebesgue measure, then $\operatorname{pdf}_{\phi}$ is never flat at zero. For each $\phi \in \Phi$, construct now the corresponding cumulative distribution function and call the set of them $\Psi$. Notice that every $\psi \in \Psi$ must be convex, strictly decreasing, and differentiable functions, because the corresponding PDFs exist, are nondecreasing, and never flat at zero. We are left to show that $\Psi$ is pointwise compact: but this follows trivially from the standard result that for any two distributions $\phi, \phi^{\prime}$ on $[0,1]$ with corresponding CDFs $\psi$ and $\psi^{\prime}$ such that both are continuous on $[0,1]$, we have that $\phi \rightarrow \phi^{\prime}$ weakly if and only if, $\psi \rightarrow \psi^{\prime}$ pointwise. ${ }^{50}$ The desired representation then follows trivially, as does the existence of a minimal representation. Finally, the unique properties of the minimal representation follow trivially from the uniqueness properties of the representation in (3), discussed above.

(2) $\Rightarrow$ (1). We start by proving the necessity of Axiom 3 (continuity). For brevity in what follows we will only prove that if $\succeq$ admits the representation in (3), then for any $\left(p_{n}\right) \in(\Delta(X))^{\infty}$ and for any $p, q \in \Delta(X)$, if $p_{n} \succeq q$ for all $n$ and if $p_{n} \rightarrow p$ (in the topology of weak convergence), then $p \succeq q$. The proof for the specular case in which $p_{n} \preceq q$ for all $n$ is identical, while the extension to nonconstant acts follows by standard arguments once the convergence for constant acts is established. To avoid confusion, we denote $p_{n} \rightarrow^{w} p$ to indicate weak convergence, $f_{n} \rightarrow^{p} f$ to denote pointwise convergence, and $\rightarrow$ to indicate convergence in $\mathbb{R}$.

Claim 13. Consider $\psi_{n} \in \Psi^{\infty}, \psi \in \Psi, p_{n} \in \Delta(X)^{\infty}$, and $p \in \Delta(X)$ such that $\psi_{n} \rightarrow^{p} \psi$ and $p_{n} \rightarrow{ }^{w} p$. Then $\operatorname{RDU}_{u, \psi_{n}}\left(p_{n}\right) \rightarrow \operatorname{RDU}_{u, \psi}(p)$.

Proof. Consider $\psi_{n} \in \Psi^{\infty}, \psi \in \Psi, p_{n} \in \Delta(X)^{\infty}$, and $p \in \Delta(X)$ as in the statement of the claim. (What follows is an adaptation of the proofs in Chateauneuf 1999, Remark 9 to our case.) Notice that since $X$ is a connected and compact set, and since $u$ is continuous, we can assume without loss of generality (wlog) $u(X)=[0,1]$. Also, for any $t \in[0,1]$, define $A_{t}:=\{x \in X: u(x)>t\}$. Then notice that for any $p \in \Delta(X)$ and $\psi \in \Psi$ we have $\operatorname{RDU}_{u, \psi}(p)=\int_{0}^{1} \psi\left(p\left(A_{t}\right)\right) \mathrm{d} t$. Define now $H_{n}, H:[0,1] \rightarrow[0,1]$ by

${ }^{50}$ This is a standard result. See, for example, the discussion in Billingsley (1995, Chapter 5). 
$H_{n}(t)=\psi_{n}\left(p_{n}\left(A_{t}\right)\right)$ and $H(t)=\psi\left(p\left(A_{t}\right)\right)$. We then have $\operatorname{RDU}_{u, \psi_{n}}\left(p_{n}\right)=\int_{0}^{1} H_{n}(t) \mathrm{d} t$ and $\operatorname{RDU}_{u, \psi}(p)=\int_{0}^{1} H(t) \mathrm{d} t$. Since $\left|H_{n}(t)\right| \leq 1$ for all $t \in[0,1]$ and for all $n$, then by the dominated convergence theorem (see Aliprantis and Border 2006, Theorem 11.21), to prove that $\mathrm{RDU}_{u, \psi_{n}}\left(p_{n}\right) \rightarrow \mathrm{RDU}_{u, \psi}(p)$ we only need to show that $H_{n}(t) \rightarrow H(t)$ for almost all $t \in[0,1]$. To do this, we denote $M_{p}:=\{r \in[0,1]: \exists x \in \operatorname{supp}(p)$ such that $u(x)=$ $r$, and we will show that we have $H_{n}(t) \rightarrow H(t)$ for all $t \in[0,1] \backslash M_{p}$ : since $p$ is a simple lottery (with therefore finite support), this will be enough.

Consider some $t \in[0,1] \backslash M_{p}$, and notice that we must have that $A_{t}$ is a continuity set of $p$. To see why, notice that since $u$ is continuous, $A_{t}$ must be open, and we have that $\delta A_{t}=\{x \in X: u(x)=t\}$; and since $t \notin M_{t}$, then we must have $p\left(\delta A_{t}\right)=0$. By the Portmanteau theorem ${ }^{51}$ we then have $p_{n}\left(A_{t}\right) \rightarrow p\left(A_{t}\right)$. We will now argue that for any such $t$ we must also have $H_{n}(t) \rightarrow H(t)$, which will conclude the argument. To see why, consider any $t \in[0,1] \backslash M_{p}$, and notice that we must have $\left|H_{n}(t)-H(t)\right|=\mid \psi_{n}\left(p_{n}\left(A_{t}\right)\right)-$ $\psi\left(p\left(A_{t}\right)\right)|<| \psi_{n}\left(p_{n}\left(A_{t}\right)\right)-\psi_{n}\left(p\left(A_{t}\right)\right)|+| \psi_{n}\left(p\left(A_{t}\right)\right)-\psi\left(p\left(A_{t}\right)\right) \mid$. At the same time, $\left|\psi_{n}\left(p_{n}\left(A_{t}\right)\right)-\psi_{n}\left(p\left(A_{t}\right)\right)\right|$ can be made arbitrarily small since $p_{n}\left(A_{t}\right) \rightarrow p\left(A_{t}\right)$ and $\psi_{n}$ is continuous, and $\left|\psi_{n}\left(p\left(A_{t}\right)\right)-\psi\left(p\left(A_{t}\right)\right)\right|$ can be made arbitrarily small since $\psi_{n} \rightarrow^{p} \psi$. But then we must have $H_{n}(t) \rightarrow H(t)$ as sought.

Notice, therefore, that we can apply standard generalizations of Berge's theorem of the maximum, such as Aliprantis and Border (2006, Theorem 17.13), ${ }^{52}$ and therefore prove Axiom 3 (continuity).

Next, we turn to prove the necessity of Axiom 5 (hedging). To this end, let us define the notion of enumeration.

Definition 12. A simple enumeration of a lottery $q$ is a step function $x:[0,1] \rightarrow X$ such that $l(\{z \in[0,1] \mid f(z)=w\})=q(w) \forall w \in \operatorname{supp}(q)$.

Let $N(x) \in \mathbb{N}$ be the number of steps in $x$, let $x_{n}$ be the value of $f(x)$ at each step, and let $p^{x}\left(x_{n}\right)$ be the Lebesgue measure of each step $x_{n}$.

Claim 14. Let $p$ be some lottery and let $x, y$ be two simple enumerations of $p$ such that $x_{i-1} \succeq x_{i}$ for all $2 \leq i \leq n$. Then, if $\psi$ is a convex $R D U$ functional and $u$ is a utility function that represents $\succeq$, we have

$$
W(x)=\psi\left(p^{x}\left(x_{1}\right)\right) u\left(x_{1}\right)+\sum_{i=2}^{N(x)}\left(\psi\left(\sum_{j=1}^{i} p^{x}\left(x_{j}\right)\right)-\psi\left(\sum_{j=1}^{i-1} p^{x}\left(x_{j}\right)\right)\right) u\left(x_{i}\right),
$$

which must be smaller than or equal to

$$
\psi\left(p^{y}\left(y_{1}\right)\right) u\left(y_{1}\right)+\sum_{i=2}^{N(y)}\left(\psi\left(\sum_{j=1}^{i} p^{y}\left(y_{j}\right)\right)-\psi\left(\sum_{j=1}^{i-1} p^{y}\left(y_{j}\right)\right)\right) u\left(y_{i}\right)=W(y) .
$$

\footnotetext{
${ }^{51}$ See Billingsley (1995, Chapter 5).

${ }^{52}$ In particular, in our case the correspondence $\rho$ in the statement of the theorem would be constant and equal to $\Psi$, which is nonempty and compact, while the function $f$ in the statement of the theorem would correspond to the function $\operatorname{RDU}_{u, \psi}(p)$ seen as a function of both $\psi$ and $p$, which, as we have seen, is continuous.
} 
Proof. We begin by proving the claim for cases in which $p^{y}$ maps to rational numbers; then we extend the claim using the continuity of $W$. As $p^{y}\left(y_{i}\right)$ is rational, for all $i \in$ $1, \ldots, N(y)$, we can write each $p^{y}\left(y_{i}\right)=m_{i} / n_{i}$ for some set of integers $\left\{m_{i}\right\}$ and $\left\{n_{i}\right\}$. This means that there is a set of natural numbers $\left\{k_{i}\right\}$ such that $p^{y}\left(y_{i}\right)=k_{i} / \prod n_{i}$. Notice that we can rewrite the step function $y$ as a different step function $\bar{y}$ defined by the intervals $\left\{\left[j / \prod n_{i},(j+1) / \prod n_{i}\right)\right\}_{j=0}^{\prod n_{i}-1}$, where the value of the function in the interval $\left[j / \prod n_{i},(j+\right.$ 1)/ $\left.\prod n_{i}\right)$ is equal to the value of $y$ in the interval $\left[p^{y}\left(y_{l}\right), p^{y}\left(y_{m}\right)\right)$, where $l=\max \{t \in \mathbb{N} \mid$ $\left.p^{y}\left(y_{t}\right) \leq j / \prod n_{i}\right\}$ and $\min \left\{t \in \mathbb{N} \mid p^{y}\left(y_{t}\right) \geq(j+1) / \prod n_{i}\right\}$. In other words, we have split the original step function $y$ into a finite number of equally spaced steps, while preserving the value of the original function (again we can do this because the original function had steps defined by rational numbers). We can therefore now think of $\bar{y}$ as consisting of a finite number of elements of equal length that can be interchanged using the procedure we discuss below. Note that redefining $y$ in this way does not change the function, i.e., $y(t)=\bar{y}(t) \forall t$, and neither does it affect its utility, i.e., $W(y)=W(\bar{y})$.

Now order the steps of $\bar{y}$ using $\succeq$, breaking ties arbitrarily: let $\bar{y}^{1}$ denote the best step of $\bar{y}$, let $\bar{y}^{2}$ denote the next best element, and so on. We next define a sequence of enumerations and functions recursively:

(i) Let ${ }^{1} \bar{y}=\bar{y}$. Define the function ${ }^{1} r:\{1, \ldots, N(y)\} \rightarrow \mathbb{N}$ such that ${ }^{1} r(j)$ is the original position of $\bar{y}^{j}$ for all $j$ (i.e., ${ }^{1} r(j)=\left\{n \in \mathbb{N} \mid{ }^{1} \bar{y}_{i r(j)}=\bar{y}^{j}\right\}$ ).

(ii) Define ${ }^{i} \bar{y}$ as ${ }^{i} \bar{y}(t)=\bar{y}^{i}$ for $t \in\left[(i-1) / \prod n_{i}, i / \prod n_{i}\right)$, define ${ }^{i} \bar{y}(t)={ }^{i-1} \bar{y}_{i}$ for $t \in$ $\left[\left({ }^{i-1} r(i)-1\right) / \prod n_{i},{ }^{i-1} r(i) / \prod n_{i}\right)$, and define ${ }^{i} \bar{y}(t)={ }^{i=1} \bar{y}(t)$ otherwise.

(iii) Define ${ }^{i} r(j)$ as the position of $y^{j}$ in ${ }^{i} \bar{y}$ for all $j$ (i.e., ${ }^{i} r(j)=\left\{n \in \mathbb{N} \mid{ }^{i} \bar{y}_{i(j)}=\bar{y}^{j}\right\}$ ).

So, at each stage, this procedure takes the previous function, looks for the $i$ th best step of $\bar{y}$, and switches it into the $i$ th position in the enumeration (while moving whatever was in that slot back to where the best element came from). The function ${ }^{i} r$ keeps track of the location of each of the steps of $\bar{y}$ in each iteration $i$. The first thing to note is that the final element in this sequence, $\Pi n_{i} \bar{y}$, is equivalent to $x$, in the sense that $W(x)=W\left(\prod n_{i} \bar{y}\right)$ : clearly, each of these switches preserves the Lesbegue measure associated to each prize; thus, $\prod n_{i} \bar{y}$ is an enumeration of $p$. Furthermore $\prod n_{i} \bar{y}_{i-1} \succeq \prod n_{i} \bar{y}_{i}$ for all $i$ by construction, meaning that $u\left(\prod n_{i} \bar{y}(t)\right)=u(x(t))$ for all $t$.

Next we show that $W\left({ }^{i} y\right) \leq W\left({ }^{i-1} y\right)$ for all $i \in\left\{2, \ldots, \prod n_{i}\right\}$ First note that it must be the case that ${ }^{i-1} \bar{y}_{i} \preceq \bar{y}^{i}$ : in words, the $i$ th best element of $\bar{y}$ must be weakly better than whatever is in the $i$ th slot in ${ }^{i-1} \bar{y}$. To see this, note that if this were not the case, then it must be the case that ${ }^{i-1} \bar{y}_{i}=\bar{y}^{j}$ for some $j<i$. But, by the iterative procedure, $\bar{y}^{j}$ must be in slot ${ }^{i-1} \bar{y}_{j} \neq{ }^{i-1} \bar{y}_{i}$. Next note that it must be the case that ${ }^{i-1} r(i) \geq i$. By the iterative procedure, for all $j<i,{ }^{i-1} \bar{y}_{j}=\bar{y}^{j} \neq \bar{y}^{i}$. Thus, as ${ }^{i-1} r(i)$ is the location of $\bar{y}^{i}$ in ${ }^{i-1} \bar{y}_{j}$, it must be the case that ${ }^{i-1} r(i) \geq i$.

Next note that ${ }^{i} y$ and ${ }^{i-1} y$ differ only on the intervals $\left[(i-1) / \prod n_{i}, i / \prod n_{i}\right)$ and $\left[\left({ }^{i-1} r(i)-1\right) / \prod n_{i},{ }^{i-1} r(i) / \prod n_{i}\right)$. Thus, we can write the difference between $W\left({ }^{i} y\right)$ and $W\left({ }^{i-1} y\right)$ as $\left(\psi\left(\sum_{j=1}^{i} p\left({ }^{i} y_{j}\right)\right)-\psi\left(\sum_{j=1}^{i-1} p\left({ }^{i} y_{j}\right)\right)\right)\left(u\left({ }^{i-1} \bar{y}_{i}\right)-u\left(\bar{y}^{i}\right)\right)+\left(\psi\left(\sum_{j=1}^{r(i)} p\left({ }^{i} y_{j}\right)\right)-\right.$ 
$\left.\psi\left(\sum_{j=1}^{r(i)-1} p\left({ }^{i} y_{j}\right)\right)\right)\left(u\left(\bar{y}_{i}\right)-u\left({ }^{i-1} \bar{y}_{i}\right)\right)$. This is equal to

$$
\left(\left(\psi\left(\frac{i}{\prod n_{i}}\right)-\psi\left(\frac{i-1}{\prod n_{i}}\right)\right)-\left(\psi\left(\frac{{ }^{i-1} r(i)}{\prod n_{i}}\right)-\psi\left(\frac{{ }^{i-1} r(i)-1}{\prod n_{i}}\right)\right)\right)\left(u\left({ }^{i-1} \bar{y}_{i}\right)-u\left(\bar{y}^{i}\right)\right) .
$$

Now, as ${ }^{i-1} \bar{y}_{i} \preceq \bar{y}^{i}$, it must be the case that $u\left({ }^{i-1} \bar{y}_{i}\right) \leq u\left(\bar{y}^{i}\right)$, and so $\left(u\left({ }^{i-1} \bar{y}_{i}\right)-\right.$ $\left.u\left(\bar{y}^{i}\right)\right) \leq 0$. Furthermore, it must be the case that the term in the first parentheses is also weakly negative by the convexity of $\psi$. To see this, define the function $\bar{\psi}(x)=$ $\psi\left(x+(i-1) / \prod n_{i}\right)-\psi\left((i-1) / \prod n_{i}\right)$. This is a convex function with $\bar{\psi} \geq 0$ and so it is subadditive. This means that we have $\bar{\psi}\left({ }^{i-1} r(i) / \prod n_{i}-(i-1) / \prod n_{i}\right) \geq \bar{\psi}\left(\left({ }^{i-1} r(i)-1\right) / \prod n_{i}-\right.$ $\left.(i-1) / \prod n_{i}\right)+\bar{\psi}\left(\left({ }^{i-1} r(i) / \prod n_{i}-(i-1) / \prod n_{i}\right)-\left(\left({ }^{i-1} r(i)-1\right) / \prod n_{i}-(i-1) / \prod n_{i}\right)\right)$. In turn, this is equal to $\bar{\psi}\left(\left({ }^{i-1} r(i)-1\right) / \prod n_{i}-(i-1) / \prod n_{i}\right)+\bar{\psi}\left(1 / \prod n_{i}\right)$. If we then substitute the original function, we get $\psi\left({ }^{i-1} r(i) / \prod n_{i}\right) \geq \psi\left(\left({ }^{i-1} r(i)-1\right) / \prod n_{i}\right)+\psi\left(i / \prod n_{i}\right)-$ $\psi\left((i-1) / \prod n_{i}\right)$. Thus, by iteration we have $W(y)=W(\bar{y})=W\left({ }^{1} \bar{y}\right) \geq W\left(\prod n_{i} \bar{y}(t)\right)=W(x)$ and we are done.

To extend the proof to enumerations with irrational $p$ functions, take such a function $y$ and associated $x$ that is the rank order enumeration of $y$, whereby $p^{y}\left(y_{i}\right)$ is not guaranteed to be rational for all $i \in 1, \ldots, N(y)$. Now note that $p^{y}$ is a vector in $\mathbb{R}^{N(y)}$. Note that we can construct a sequence of vectors $q^{i} \in \mathbb{Q}^{N(y)}$ such that $\left\{q^{i}\right\} \rightarrow p^{y}$. Define the simple enumeration $y^{i}$ as the step function whereby $y^{i}(t)=y_{n}$ for $t \in\left[\sum_{j=0}^{n-1} q_{i-1}^{i}, \sum_{j=0}^{n} q_{i-1}^{i}\right)$. The utility of the enumeration $y^{i}$ is given by $W\left(y^{i}\right)=\psi\left(q_{1}^{i}\right) u\left(y_{1}\right)+\sum_{k=2}^{N(y)}\left(\psi\left(\sum_{j=0}^{k} q_{i-1}^{i}\right)-\right.$ $\left.\psi\left(\sum_{j=0}^{k-1} q_{i-1}^{i}\right)\right) u\left(y_{k}\right)$.

As $q_{j}^{i} \rightarrow p^{y}\left(y_{j}\right)$ and as $\psi$ is continuous, then it must be the case that $W\left(y^{i}\right) \rightarrow W(y)$. Similarly, if we let $x^{i}$ be the rank enumeration of $y^{i}$, then it must be the case that $W\left(x^{i}\right) \rightarrow$ $W(x)$. Thus, if it were the case that $W(y)>W(x)$, then there would be some $i$ such that $W\left(y^{i}\right)>W\left(x^{i}\right)$. But as $y^{i}$ is rational, this contradicts the above result.

We now turn to prove that Axiom 5 is satisfied. Again we will prove this only for degenerate acts, the extension to the general case being trivial. Let $p$ and $q$ be two lotteries such that $p \sim q$ and $r \in p \oplus q$. Let $x$ be the enumeration of $r$. Then there must be two enumerations $z^{x}$ and $z^{y}$ such that (i) $z_{i}=\frac{1}{2} z_{i}^{x} \oplus \frac{1}{2} z_{i}^{y}$ for all $i$, and (ii) for every $x_{i}, \sum_{i \mid z_{i}^{x}=x_{i}} r\left(z_{i}\right)=p\left(x_{i}\right)$ and $\sum_{i \mid z_{i}^{y}=y_{i}} r\left(z_{i}\right)=p\left(y_{i}\right)$. Now, the utility of $r$ is given by $U(r)=\min _{\pi \in \Pi} \sum_{i}\left(\pi\left(\sum_{j=0}^{i-1} r\left(z_{j}\right)\right)-\pi\left(\sum_{j=0}^{i} r\left(z_{j}\right)\right)\right) u\left(z_{i}\right)$, which is equal to $\min _{\pi \in \Pi} \sum_{i}\left(\pi\left(\sum_{j=0}^{i-1} r\left(z_{j}\right)\right)-\pi\left(\sum_{j=0}^{i} r\left(z_{j}\right)\right)\right)\left(\frac{1}{2}\left(u\left(z_{i}^{x}\right)+u\left(z_{i}^{y}\right)\right)\right)$, which is, in turn, equal to $\min _{\pi \in \Pi}\left[\frac{1}{2}\left(\sum_{i}\left(\pi\left(\sum_{j=0}^{i-1} r\left(z_{j}\right)\right)-\pi\left(\sum_{j=0}^{i} r\left(z_{j}\right)\right)\right) u\left(z_{i}^{x}\right)\right)+\frac{1}{2}\left(\sum_{i}\left(\pi\left(\sum_{j=0}^{i-1} r\left(z_{j}\right)\right)-\right.\right.\right.$ $\left.\left.\left.\pi\left(\sum_{j=0}^{i} r\left(z_{j}\right)\right)\right) u\left(z_{i}^{y}\right)\right)\right]$. This must be larger than or equal to $\frac{1}{2} \min _{\pi \in \Pi}\left(\sum_{i}\left(\pi\left(\sum_{j=0}^{i-1} r\left(z_{j}\right)\right)-\right.\right.$ $\left.\left.\pi\left(\sum_{j=0}^{i} r\left(z_{j}\right)\right)\right) u\left(z_{i}^{x}\right)\right)+\frac{1}{2} \min _{\pi \in \Pi}\left(\sum_{i}\left(\pi\left(\sum_{j=0}^{i-1} r\left(z_{j}\right)\right)-\pi\left(\sum_{j=0}^{i} r\left(z_{j}\right)\right)\right) u\left(z^{y}\right)\right)$.

Note that the enumerations are not in rank order, but by Claim 14, reordering can only decrease the utility of the enumeration by shuffling them into the rank order for every $\pi \in \Pi$. Let $\bar{z}^{x}$ and $\bar{z}^{y}$ be the rank order enumerations of $z^{x}$. We must then have that $U(r)$ is larger than or equal to $\frac{1}{2} \min _{\pi \in \Pi}\left(\sum_{i}\left(\pi\left(\sum_{j=0}^{i-1} r\left(z_{j}\right)\right)-\pi\left(\sum_{j=0}^{i} r\left(z_{j}\right)\right)\right) u\left(z_{i}^{x}\right)\right)+$ $\frac{1}{2} \min _{\pi \in \Pi}\left(\sum_{i}\left(\pi\left(\sum_{j=0}^{i-1} r\left(z_{j}\right)\right)-\pi\left(\sum_{j=0}^{i} r\left(z_{j}\right)\right)\right) u\left(z^{y}\right)\right)$. This is larger than or equal to 
$\frac{1}{2} \min _{\pi \in \Pi}\left(\sum_{i}\left(\pi\left(\sum_{j=0}^{i-1} r\left(z_{j}\right)\right)-\pi\left(\sum_{j=0}^{i} r\left(z_{j}\right)\right)\right) u\left(\bar{z}_{i}^{x}\right)\right)+\frac{1}{2} \min _{\pi \in \Pi}\left(\sum_{i}\left(\pi\left(\sum_{j=0}^{i-1} r\left(z_{j}\right)\right)-\right.\right.$ $\left.\left.\pi\left(\sum_{j=0}^{i} r\left(z_{j}\right)\right)\right) u\left(\bar{z}_{i}^{y}\right)\right)$, which is then equal to $\frac{1}{2} U(p)+\frac{1}{2} U(q)$ as sought.

We now turn to Axiom 1 (FOSD). Let $\pi$ be a continuous RDU functional. We know (e.g., Wakker 1994, Theorem 12) that it respects FOSD. Thus, suppose that $p$ first order stochastically dominates $q$ and let $\pi^{*} \in \Pi$ be the functional that minimizes the utility of $p$. We know that the utility of $q$ under this functional has to be lower than the utility of $p$; thus, the utility of $q$ (which is assessed under the functional that minimizes the utility of $q$ ) is lower than that of $p$.

Finally, Axiom 2 (monotonicity), Axiom 4 (objective trade-off consistency), and Axiom 6 (degenerate independence) follow from standard arguments.

Proof of Proposition 1. Notice first of all that $\oplus_{\succeq_{1}}=\oplus_{\succeq_{2}}$ holds if and only if $u_{1}$ is a positive affine transformation of $u_{2}$. Also, define by $V_{1}$ and $V_{2}$ the functionals corresponding to the minimal MP-MW representations of $\succeq_{1}$ and $\succeq_{2}$, respectively.

(1) $\Rightarrow$ (2). Suppose now that we have that $\succeq_{2}$ is more attracted to certainty than $\succeq_{1}$. Since $\oplus_{\succeq_{1}}=\oplus_{\succeq_{2}}$, then $u_{1}$ is a positive affine transformation of $u_{2}$ and let us assume first that $u_{1}=u_{2}$. Now consider $p \in \Delta(X)$ and $x_{1}, x_{2} \in X$ such that $p \sim_{1} \delta_{x_{1}}$ and $p \sim_{2}$ $\delta_{x_{2}}$. Notice that since $\succeq_{2}$ is more attracted to certainty than $\succeq_{1}$, then $\delta_{x_{1}} \succeq_{1} \delta_{x_{2}}$; thus, $u_{1}\left(x_{1}\right) \geq u_{1}\left(x_{2}\right)$. Thus, we must have $V_{1}(p)=u_{1}\left(x_{1}\right) \geq u_{1}\left(x_{2}\right)=u_{2}\left(x_{2}\right)=V_{2}(p)$. Thus, we must have

$$
\min _{\psi \in \Psi_{1}} \operatorname{RDU}_{u_{1}, \psi}(p) \geq \min _{\psi \in \Psi_{2}} \operatorname{RDU}_{u_{1}, \psi}(p) .
$$

We will now argue that this implies $\Psi_{1} \subseteq \Psi_{2}$. Suppose not, i.e., that there exists $\psi^{\prime} \in$ $\Psi_{1} \backslash \Psi_{2}$. But then, by the above discussion, we must have that

$$
\min _{\psi \in \Psi_{2}} \operatorname{RDU}_{u_{1}, \psi}(p)=\min _{\psi \in \Psi_{2} \cup\left\{\psi^{\prime}\right\}} \operatorname{RDU}_{u_{1}, \psi}(p),
$$

violating the uniqueness properties of the minimal MP-MW representation.

Similarly, consider $f \in \mathcal{F}$ such that $f(\omega)=\delta_{x}$ for some $x \in X$. Consider $x_{1}^{\prime}, x_{2}^{\prime} \in X$ such that $f \sim_{1} \delta_{x_{1}^{\prime}}$ and $f \sim_{2} \delta_{x_{2}^{\prime}}$. Notice that since $\succeq_{2}$ is more attracted to certainty than $\succeq_{1}$, then $\delta_{x_{1}^{\prime}} \succeq_{1} \delta_{x_{2}^{\prime}}$; thus, $u_{1}\left(x_{1}^{\prime}\right) \geq u_{1}\left(x_{2}^{\prime}\right)$. Thus, we must have $V_{1}(f)=u_{1}\left(x_{1}^{\prime}\right) \geq u_{1}\left(x_{2}^{\prime}\right)=$ $u_{2}\left(x_{2}^{\prime}\right)=V_{2}(f)$. Thus, we must have

$$
\min _{\pi \in \Pi_{1}} \sum \pi(\omega) u_{1}(f(\omega)) \geq \min _{\pi \in \Pi_{2}} \sum \pi(\omega) u_{2}(f(\omega)) .
$$

We will now argue that this implies $\Pi_{1} \subseteq \Pi_{2}$. Suppose not, i.e., that there exists $\pi^{\prime} \in$ $\Pi_{1} \backslash \Pi_{2}$. But then, by the above discussion, we must have that

$$
\min _{\pi \in \Pi_{2}} \sum \pi(\omega) u_{2}(f(\omega)) \geq \min _{\pi \in \Pi_{2} \cup\left\{\pi^{\prime}\right\}} \sum \pi(\omega) u_{2}(f(\omega)),
$$

violating the uniqueness properties of the minimal MP-MW representation.

This proves one direction, assuming $u_{1}=u_{2}$. It is straightforward to extend the proof to the case in which $u_{1}$ is a positive affine transformation of $u_{2}$. 
(2) $\Rightarrow$ (1) Suppose that $u_{1}$ is a positive affine transformation of $u_{2}$, i.e., there exist $a, b \in \mathbb{R}, a>0$, such that $u_{2}=a u_{1}+b$, and $\Pi_{1} \subseteq \Pi_{2}, \Psi_{1} \subseteq \Psi_{2}$. Now notice that for all $f$ we must have

$$
\begin{aligned}
V_{2}(f) & =\min _{\pi \in \Pi_{2}} \sum \pi(\omega) \min _{\psi \in \Psi_{2}} \operatorname{RDU}_{u_{2}, \psi}(f(\omega)) \\
& \leq \min _{\pi \in \Pi_{1}} \sum \pi(\omega) \min _{\psi \in \Psi_{1}} \operatorname{RDU}_{u_{2}, \psi}(f(\omega))=a V_{1}(f)+b .
\end{aligned}
$$

At the same time, we have that $V_{2}\left(\delta_{x}\right)=u_{2}(x)=a u_{1}(x)+b=a V_{1}\left(\delta_{x}\right)+b$. Thus, we must have that $\succeq_{2}$ is more attracted to certainty than $\succeq_{1}$.

Proof of Proposition 2. Consider a preference relation $\succeq$ that satisfies Axioms 1-3 as well as Axiom 7. By the latter, there must exist $\pi \in \Delta(\Omega)$ such that $f^{\pi} \succeq f$ for all $f \in \mathcal{F}$. Now define $\succeq_{\text {an }}$ by

$$
p \succeq \text { an } q \quad \Leftrightarrow \quad p \succeq q
$$

for all $p, q \in \Delta(X)$, and by

$$
f^{\pi} \sim f
$$

for all $f \in \mathcal{F}$. By Axioms 1-3, this preference is well defined, and it is ambiguity neutral by construction. Now consider $p \in \Delta(X)$ and $f \in \mathcal{F}$ such that $p \succeq_{\text {an }}\left(\succ_{\text {an }}\right) f$. Then notice that by construction we must have $f \sim_{\text {an }} f^{\pi}$; hence, $p \succeq_{\text {an }}\left(\succ_{\text {an }}\right) f^{\pi}$. By (3), we must therefore have $p \succeq(\succ) f^{\pi}$. But we know that, by Axiom 7, $f^{\pi} \succeq f$; hence, $p \succeq(\succ) f$, proving that $\succeq$ is ambiguity averse.

Conversely, consider a preference relation $\succeq$ that satisfies Axioms 1-3 and that is ambiguity averse. Then there must exists $\succeq_{\text {an }}$ that is ambiguity neutral and such that $\succeq$ is more ambiguity averse than $\succeq_{\text {an }}$. Since $\succeq_{\text {an }}$ is ambiguity neutral, then there must exist some $\pi \in \Delta(\Omega)$ such that $f^{\pi} \sim_{\text {an }} f$ for all $f \in \mathcal{F}$. Since $\succeq$ is more ambiguity averse than $\succeq_{\text {an }}$, then $f^{\pi} \sim_{\text {an }} f$ implies $f^{\pi} \succeq f$. Since this holds for all $f \in \mathcal{F}, \succeq$ satisfies Axiom 7 .

Proof of Theorem 2. (1) $\Rightarrow$ (2). Consider a preference relation that admits a multiple priors-multiple weighting representation $(u, \Pi, \Psi)$ and suppose that it satisfies Axiom 7 . Then there must exist some $\pi \in \Delta(\Omega)$ such that $f^{\pi} \succeq f$ for all $f \in \mathcal{F}$. We will now prove that $\Pi \supseteq \Gamma_{\Psi}(\pi)$. Suppose this is not the case: there exists a prior $\pi^{\prime}$ on $\Delta(\Omega)$ such that $\bar{\pi} \in \Gamma_{\Psi}(\pi)$ and $\bar{\pi} \notin \Pi$. Then there must exist some $f \in \mathcal{F}$ such that $\sum \bar{\pi}(\omega) U(f(\omega))<$ $\min _{\pi^{\prime} \in \Pi} \sum \pi^{\prime}(\omega) U(f(\omega))$; otherwise the multiple priors-multiple weighting representation $(u, \Pi \cup\{\bar{\pi}\}, \Psi)$ would represent the same preferences, violating the uniqueness properties of the representation. Notice also that since all probability weightings $\psi \in \Psi$ are convex, we must also have that $\operatorname{RDU}_{u, \psi}\left(f^{\pi}\right)=\min _{l \in \mathcal{L}} \sum \gamma_{\psi, l}(\pi)(\omega) U(f(\omega))$; thus,

$$
\begin{aligned}
V\left(f^{\pi}\right) & =\min _{\psi \in \Psi} \operatorname{RDU}_{u, \psi}\left(f^{\pi}\right)=\min _{l \in \mathcal{L}, \psi \in \Psi} \sum \gamma_{\psi, l}(\pi)(\omega) U(f(\omega)) \\
& =\min _{\pi^{\prime} \in \Gamma_{\Psi}(\pi)} \sum \pi^{\prime}(\omega) U(f(\omega)) \leq \sum \bar{\pi}(\omega) U(f(\omega)) \\
& <\min _{\pi^{\prime} \in \Pi} \sum \pi^{\prime}(\omega) U(f(\omega))=V(f) .
\end{aligned}
$$

Thus, $f^{\pi} \prec f$, contradicting Axiom 7 . 
(2) $\Rightarrow(\mathbf{1})$. Consider a preference relation that admits a multiple priors-multiple weighting representation $(u, \Pi, \Psi)$ such that $\Pi \supseteq \Gamma_{\Psi}(\pi)$ for some $\pi \in \Pi$. We will now prove $f^{\pi} \succeq f$ for all $f \in \mathcal{F}$. Consider $f \in \mathcal{F}$ and any enumeration $l \in \mathcal{L}$ such that $f\left(l^{-1}(i-1)\right) \succeq f\left(l^{-1}(i)\right)$ for $i=2, \ldots,|\Omega|$. Notice that by definition of $\gamma_{\psi, l}$ we must have $\operatorname{RDU}_{u, \psi}\left(f^{\pi}\right)=\sum \gamma_{\psi, l}(\pi)(\omega) U(f(\omega))$. Thus,

$$
\begin{aligned}
V\left(f^{\pi}\right) & =\min _{\psi \in \Psi} \operatorname{RDU}_{u, \psi}\left(f^{\pi}\right)=\min _{\psi \in \Psi} \sum \gamma_{\psi, l}(\pi)(\omega) U(f(\omega)) \\
& \geq \min _{l^{\prime} \in \mathcal{L}, \psi \in \Psi} \sum \gamma_{\psi, l^{\prime}}(\pi)(\omega) U(f(\omega)) \\
& =\min _{\pi^{\prime} \in \Gamma_{\Psi}(\pi)} \sum \pi^{\prime}(\omega) U(f(\omega))=V(f) .
\end{aligned}
$$

Thus, $f^{\pi} \succeq f$.

\section{REFERENCES}

Abdellaoui, Mohammed (2002), "A genuine rank-dependent generalization of the Von Neumann-Morgenstern expected utility theorem.” Econometrica, 70, 717-736. [386, 388]

Abdellaoui, Mohammed, Aurélien Baillon, Laetitia Placido, and Peter P. Wakker (2011), "The rich domain of uncertainty: Source functions and their experimental implementation." American Economic Review, 101, 695-723. [396]

Agranov, Marina and Pietro Ortoleva (2017), "Stochastic choice and preferences for randomization.” Journal of Political Economy, 125, 40-68. [396]

Aliprantis, Charalambos D. and Kim C. Border (2006), Infinite Dimensional Analysis. Springer, Berlin. [414, 415]

Aliprantis, Charalambos D. and Owen Burkinshaw (2006), Positive Operators. Springer, The Netherlands. [409]

Anscombe, Francis J. and Robert J. Aumann (1963), "A definition of subjective probability." Annals of Mathematical Statistics, 34, 199-205. [378, 380, 394, 395, 397, 398]

Billingsley, Patrick (1995), Probability and Measure. Wiley, New York. [411, 414, 415]

Casadesus-Masanell, Ramon, Peter Klibanoff, and Emre Ozdenoren (2000a), "Maxmin expected utility over Savage acts with a set of priors." Journal of Economic Theory, 92, 35-65. [381, 397]

Casadesus-Masanell, Ramon, Peter Klibanoff, and Emre Ozdenoren (2000b), "Maxmin expected utility through statewise combinations." Economics Letters, 66, 49-54. [381, 397]

Cerreia-Vioglio, Simone (2009), "Maxmin expected utility on a subjective state space: Convex preferences under risk.” Unpublished paper, Columbia University. [396] 
Cerreia-Vioglio, Simone, David Dillenberger, and Pietro Ortoleva (2015), "Cautious expected utility and the certainty effect." Econometrica, 83, 693-728. [396]

Cerreia-Vioglio, Simone, David Dillenberger, Pietro Ortoleva, and Gil Riella (2017), "Deliberately stochastic." Unpublished paper, Columbia University. [396]

Cerreia-Vioglio, Simone, Paolo Ghirardato, Fabio Maccheroni, Massimo Marinacci, and Marciano Siniscalchi (2011a), "Rational preferences under ambiguity." Economic Theory, 48, 341-375. [395]

Cerreia-Vioglio, Simone, Fabio Maccheroni, Massimo Marinacci, and Luigi Montrucchio (2011b), “Uncertainty averse preferences.” Journal of Economic Theory, 146, 12751330. [395]

Chateauneuf, Alain (1999), "Comonotonicity axioms and rank-dependent expected utility theory for arbitrary consequences." Journal of Mathematical Economics, 32, 21-45. $[386,388,414]$

Chateauneuf, Alain, Fabio Maccheroni, Massimo Marinacci, and Jean-Marc Tallon (2005), "Monotone continuous multiple priors." Economic Theory, 26, 973-982. [381, $408,409]$

Chew, Soo Hong and Edi Karni (1994), "Choquet expected utility with a finite state space: Commutativity and act-independence." Journal of Economic Theory, 62, 469-479. [381, 397]

Chew, Soo Hong and Jacob S. Sagi (2008), "Small worlds: Modeling attitudes toward sources of uncertainty." Journal of Economic Theory, 139, 1-24. [396]

Dean, Mark and Pietro Ortoleva (2017), "Is it all connected? A testing ground for unified theories of behavioral economics phenomena.” Unpublished paper, Columbia University. [378]

Diecidue, Enrico and Peter P. Wakker (2001), "On the intuition of rank-dependent utility." Journal of Risk and Uncertainty, 23, 281-298. [387]

Diecidue, Enrico, Ulrich Wu, and Horst Zank (2009), "Parametric weighting functions." Journal of Economic Theory, 144, 1102-1118. [388]

Dillenberger, David (2010), "Preferences for one-shot resolution of uncertainty and Allais-type behavior." Econometrica, 78, 1973-2004. [396]

Drapeau, Samuel and Micheal Kupper (2013), "Risk preferences and their robust representation." Mathematics of Operations Research, 38, 28-62. [378, 379, 395]

Dwenger, Nadja, Dorothea Kübler, and Georg Weizsäcker (2015), "Flipping a coin: Theory and evidence.” WZB Discussion Paper, No. SP II 2013-201r. [396]

Epstein, Larry G. (1999), "A definition of uncertainty aversion.” Review of Economic Studies, 66, 579-608. [379, 394]

Ghirardato, Paolo (2004), "Defining ambiguity and ambiguity attitude." In Uncertainty in Economic Theory: Essays in Honor of David Schmeidler's 65th Birthday (Itzhak Gilboa, 
ed.), volume 63 of Routledge Frontiers of Political Economy, 36-45, Routledge, London. [389, 392]

Ghirardato, Paolo, Fabio Maccheroni, Massimo Marinacci, and Marciano Siniscalchi (2001), "A subjective spin on roulette wheels." Social Science Working Paper 1127, California Insitute of Technology. [382, 383, 395, 397]

Ghirardato, Paolo, Fabio Maccheroni, Massimo Marinacci, and Marciano Siniscalchi (2003), "A subjective spin on roulette wheels." Econometrica, 71, 1897-1908. [378, 379, $381,382,385,390,395,397,402,406]$

Ghirardato, Paolo and Massimo Marinacci (2001), "Risk, ambiguity, and the separation of utility and beliefs." Mathematics of Operations Research, 26, 864-890. [390, 402, 403, 405]

Ghirardato, Paolo and Massimo Marinacci (2002), "Ambiguity made precise: A comparative foundation." Journal of Economic Theory, 102, 251-289. [391, 392, 393, 394]

Ghirardato, Paolo and Marciano Siniscalchi (2012), "Ambiguity in the small and in the large." Econometrica, 80, 2827-2847. [395]

Gilboa, Itzhak and Massimo Marinacci (2016), "Ambiguity and the Bayesian paradigm.” In Readings in Formal Epistemology, 385-439, Springer, Cham. [395]

Gilboa, Itzhak and David Schmeidler (1989), "Maxmin expected utility with non-unique prior." Journal of Mathematical Economics, 18, 141-153. [377, 379, 385, 387, 406]

Gonzalez, Richard and George Wu (1999), "On the shape of the probability weighting function." Cognitive Psychology, 38, 129-166. [388]

Gul, Faruk (1991), “A theory of disappointment aversion.” Econometrica, 59, 667-686. [382]

Gul, Faruk (1992), “Savagés theorem with a finite number of states.” Journal of Economic Theory, 57, 99-110. [381, 397]

Gumen, Anna, Efe A. Ok, and Andrei Savochkin (2014), "Decision-making under subjective risk: Toward a general theory of pessimism." Unpublished paper, New York University. [385, 398]

Klibanoff, Peter, Massimo Marinacci, and Sujoy Mukerji (2005), "A smooth model of decision making under ambiguity.” Econometrica, 73, 1849-1892. [378, 379, 396]

Köbberling, Veronika and Peter P. Wakker (2003), "Preference foundations for nonexpected utility: A generalized and simplified technique." Mathematics of Operations Research, 28, 395-423. [381, 386, 397]

Maccheroni, Fabio (2002), “Maxmin under risk.” Economic Theory, 19, 823-831. [396]

Maccheroni, Fabio, Massimo Marinacci, and Aldo Rustichini (2006), "Ambiguity aversion, robustness, and the variational representation of preferences." Econometrica, 74, 1447-1498. [397] 
Machina, Mark J. (1985), "Stochastic choice functions generated from deterministic preferences over lotteries.” The Economic Journal, 95, 575-594. [396]

Machina, Mark J. and David Schmeidler (1992), "A more robust definition of subjective probability." Econometrica, 60, 745-780. [393, 394]

Machina, Mark J. and David Schmeidler (1995), "Bayes without Bernoulli: Simple conditions for probabilistically sophisticated choice." Journal of Economic Theory, 67, 106128. [393]

Nakamura, Yutaka (1995), "Rank dependent utility for arbitrary consequence spaces." Mathematical Social Sciences, 29, 103-129. [386]

Ok, Efe A. (2011), "Elements of Order Theory." Unpublished book, New York University. [411]

Ok, Efe A., Pietro Ortoleva, and Gil Riella (2012), "Incomplete preferences under uncertainty: Indecisiveness in beliefs versus tastes.” Econometrica, 80, 1791-1808. [392]

Quiggin, John (1982), “A theory of anticipated utility.” Journal of Economic Behavior and Organization, 3, 323-343. [379, 386]

Safra, Zvi and Uzi Segal (1998), “Constant risk aversion.” Journal of Economic Theory, 83, 19-42. [396]

Savage, Leonard J. (1954), The Foundations of Statistics. Wiley, New York. [378, 395]

Schmeidler, David (1989), "Subjective probability and expected utility without additivity.” Econometrica, 57, 571-587. [378, 379, 385, 388, 397]

Segal, Uzi (1987a), "The Ellsberg paradox and risk aversion: An anticipated utility approach." International Economic Review, 28, 175-202. [379, 397]

Segal, Uzi (1987b), "Some remarks on Quiggin's anticipated utility." Journal of Economic Behavior \& Organization, 8, 145-154. [387]

Segal, Uzi (1990), “Two-stage lotteries without the reduction axiom.” Econometrica, 58, 349-377. [379, 397]

Sopher, Barry and J. Mattison Narramore (2000), "Stochastic choice and consistency in decision making under risk: An experimental study." Theory and Decision, 48, 323-349. [396]

Starmer, Chris (2000), "Developments in non-expected utility theory: The hunt for a descriptive theory of choice under risk." Journal of Economic Literature, 38, 332-382. [386]

Tversky, Amos and Craig R. Fox (1995), "Weighing risk and uncertainty." Psychological Review, 102, 269-283. [379, 389]

Tversky, Amos and Daniel Kahneman (1992), "Advances in prospect theory: Cumulative representation of uncertainty." Journal of Risk and Uncertainty, 5, 297-323. [386] 
Wakker, Peter P. (1994), "Separating marginal utility and probabilistic risk aversion." Theory and decision, 36, 1-44. [381, 386, 397, 418]

Wakker, Peter P. (2001), “Testing and characterizing properties of nonadditive measures through violations of the sure-thing principle." Econometrica, 69, 1039-1059. [378, 379, $386,388,389,397]$

Wakker, Peter P. (2010), Prospect Theory: For Risk and Ambiguity. Cambridge University Press, Cambridge, UK. [378, 386]

Co-editor Faruk Gul handled this manuscript.

Manuscript received 9 September, 2014; final version accepted 4 September, 2015; available online 3 February, 2016. 